\title{
A new mapped WENO scheme using order-preserving mapping
}

\author{
Ruo Li ${ }^{\mathrm{a}}$, Wei Zhong $\mathrm{g}^{\mathrm{b}, \mathrm{c}, *}$ \\ ${ }^{a}$ CAPT, LMAM and School of Mathematical Sciences, Peking University, Beijing 100871, China \\ ${ }^{b}$ School of Mathematical Sciences, Peking University, Beijing 100871, China \\ ${ }^{c}$ Northwest Institute of Nuclear Technology, Xi'an 710024, China
}

\begin{abstract}
Existing mapped WENO schemes can hardly prevent spurious oscillations while preserving high resolutions at long output times. We reveal in this paper the essential reason for such phenomena. It is actually caused by that the mapping function in these schemes can not preserve the order of the nonlinear weights of the stencils. The nonlinear weights may be increased for non-smooth stencils and be decreased for smooth stencils. It is then indicated to require the set of mapping functions to be Order-Preserving in mapped WENO schemes. Therefore, we propose a new mapped WENO scheme with a set of mapping functions to be order-preserving which exhibits a remarkable advantage over the mapped WENO schemes in references. For long output time simulations, the new scheme has the capacity to attain high resolutions and avoid spurious oscillations near discontinuities meanwhile.
\end{abstract}

Keywords: mapped WENO, Order-preserving Mapping, Hyperbolic Problems

\section{Introduction}

Many numerical methods have been studied to solve the hyperbolic problems which may generate discontinuities as time evolves in its solution even if the initial condition is smooth, especially for nonlinear cases. As the discontinuities often cause spurious oscillations in numerical calculations, it is very difficult to design high order numerical schemes. Thus, the essentially non-oscillatory (ENO) schemes [12, 14, 13, 11] and weighted ENO (WENO) schemes [30, 31, 22, 17, 29] have developed quite successfully in recent decades, and they are very popular methods to solve the hyperbolic conservation laws because of their success to the ENO property. The goal of this paper is to devise a new version of the fifth-order finite volume WENO scheme for solving the following hyperbolic conservation laws

$$
\left\{\begin{array}{l}
\frac{\partial \mathbf{u}}{\partial t}+\sum_{\alpha=1}^{d} \frac{\partial \mathbf{f}_{\alpha}(\mathbf{u})}{\partial x_{\alpha}}=0, \quad x_{\alpha} \in \mathbb{R}, t>0 \\
\mathbf{u}(\mathbf{x}, 0)=\mathbf{u}_{0}(\mathbf{x})
\end{array}\right.
$$

with proper boundary conditions. Here, $\mathbf{u}=\left(u_{1}, \cdots, u_{m}\right) \in \mathbb{R}^{m}$ are the conserved variables and $\mathbf{f}_{\alpha}: \mathbb{R}^{m} \rightarrow \mathbb{R}^{m}$, $\alpha=1,2, \cdots, d$ are the Cartesian components of flux.

The first WENO scheme was developed by Liu et al. [22] in the finite volume version. It converts an $r$ th-order ENO scheme [12, 14, 13] into an $(r+1)$ th-order WENO scheme through a convex combination of all candidate substencils instead of only using the optimal smooth candidate stencil by the original ENO scheme. By introducing a different definition of the smoothness indicators used to measure the smoothness of the numerical solutions on substencils, the classic $(2 r-1)$ th-order WENO-JS scheme was proposed by Jiang and Shu [17]. The WENO-JS scheme has been successfully used in a wide number of applications. The WENO methodology within the general framework of smoothness indicators and nonlinear weights proposed in the WENO-JS scheme [17] is still in development to improve the convergence rate in smooth regions and reduce the dissipation near the discontinuities $[15,8,7,2,3,16,5,1]$.

\footnotetext{
*Corresponding author

Email addresses: rli@math.pku.edu.cn (Ruo Li), zhongwei2016@pku.edu.cn (Wei Zhong)
} 
Henrick et al. [15] pointed out that the classic WENO-JS scheme was less than fifth-order for many cases such as at or near critical points of order $n_{\mathrm{cp}}=1$ in the smooth regions. Here, we refer to $n_{\mathrm{cp}}$ as the order of the critical point; e.g., $n_{\mathrm{cp}}=1$ corresponds to $f^{\prime}=0, f^{\prime \prime} \neq 0$ and $n_{\mathrm{cp}}=2$ corresponds to $f^{\prime}=0, f^{\prime \prime}=0, f^{\prime \prime \prime} \neq 0$, etc. The necessary and sufficient conditions on the nonlinear weights for optimality of the convergence rate of the fifth-order WENO schemes were derived by Henrick et al. in [15]. These conditions were reduced to a simple sufficient condition [2] which could be easily extended to the $(2 r-1)$ th-order WENO schemes [8]. Then, by designing a mapping function that satisfies the sufficient condition to achieve the optimal order of accuracy, the original mapped WENO scheme, named WENO-M, was devised by Henrick et al. [15].

Recently, Feng et al. [7] noted that, when the WENO-M scheme was used for solving the problems with discontinuities for long output times, its mapping function may amplify the effect from the non-smooth stencils leading to a potential loss of accuracy near discontinuities. To address this issue, they proposed a piecewise polynomial mapping function with two additional requirements, that is, $g^{\prime}(0)=0$ and $g^{\prime}(1)=0(g(x)$ denotes the mapping function), to the original criteria in [15]. However, the resultant WENO-PM $k$ scheme [7] may generate the non-physical oscillations near the discontinuities as shown in Fig. 8 of [8] and Figs. 3-8 of [34]. Later, Feng et al. [8] devised an improved mapping method which is referred to as $\operatorname{WENO} \operatorname{IM}(k, A)$ where $k$ is a positive even integer and $A$ a positive real number. The broader class of the improved mapping functions of the family of the WENO-IM $(k, A)$ schemes includes the mapping function of the WENO-M scheme as a special case by taking $k=2$ and $A=1$. Feng et al. indicated that by taking $k=2$ and $A=0.1$ in the fifth-order $\operatorname{WENO} \operatorname{IM}(k, A)$ scheme, far better numerical solutions with less dissipation and higher resolution could be obtained than that of the fifth-order WENO-M scheme. However, the possible over-amplification of the contributions from non-smooth stencils exists for the WENO-IM $(k, A)$ scheme as the first derivative of its mapping function satisfies $\left(g^{\mathrm{IM}}\right)_{s}^{\prime}(0 ; k, A)=1+\frac{1}{A d_{s}^{k-1}}$, and this excessive amplification of the weights of non-smooth stencils causes spurious oscillations to occur in the solution which may even render the algorithm unstable [33], especially for higher order cases. The numerical experiments in [34] showed that the seventh- and ninth- order WENO-IM $(2,0.1)$ schemes generated evident spurious oscillations near discontinuities when the output time is large. In addition, our calculations as shown in Figs. 14 20 of this paper indicate that, even for the fifth-order WENO-IM $(2,0.1)$ scheme, the spurious oscillations are also produced when the grid number increases or a different initial condition is used.

Many other improved mapped WENO schemes, such as the WENO-PPMn [21], WENO-RM(mn0) [34], WENOMAIM $i$ [19], WENO-ACM [20] schemes and et al., have been successfully developed to improve the performances of the classic WENO-JS scheme in some ways, like achieving optimal convergence orders near critical points in smooth regions, having lower numerical dissipations and achieving higher resolutions near discontinuities, and we refer to the references for more details. However, as mentioned in previously published works of literature [8, 34], most of the existing improved mapped WENO schemes could not prevent the generation of the spurious oscillations near discontinuities, especially for long output time simulations.

Taking a long output time simulation of the linear advection problem with discontinuities as an example, we make a further study of the nonlinear weights of the existing mapped WENO schemes developed in [7, 8, 19] and the MIPWENO-ACM $k$ scheme, which is a generalized form of the WENO-ACM scheme [20] (see details in subsection 3.1). We find that, in many points, the order of the nonlinear weights for the substencils of the same global stencil has been changed in the mapping process of all these considered mapped WENO schemes. The order change of the nonlinear weights is caused by weights increasing of non-smooth substencils and weights decreasing of smooth substencils. Through theoretical analysis and extensive numerical tests, we reveal that this is the essential cause of the potential loss of accuracy of the WENO-M scheme and the spurious oscillation generation of the existing improved mapped WENO schemes.

Indicated by the observation above, we give the definition of the order-preserving (see Definition 2 below) mapping and suggest it as an additional criterion in the design of the mapping function. Then we propose a new mapped WENO scheme, referred to as MOP-WENO-ACM $k$ below, with its mapping function satisfying the additional criterion. This new version of the mapped WENO scheme achieves the optimal convergence order of accuracy even at critical points. It also has low numerical dissipation and high resolution but does not generate spurious oscillation near discontinuities even if the output time is large.

At first, a series of accuracy tests show the capacity of the proposed scheme to achieve the optimal convergence order in smooth regions with first-order critical points and its advantage in long output time simulations of the prob- 
lems with very high-order critical points. Some linear advection examples with long output times are then presented to demonstrate that the proposed scheme can obtain high resolution and does not generate spurious oscillation near discontinuities. At last, some benchmark problems modeled via the two-dimensional Euler equations are computed by various considered WENO schemes to compare with the proposed scheme. It is clear that the proposed scheme exhibits significant advantages in preventing spurious oscillations.

The organization of this paper is as follows. Preliminaries to understand the finite volume method and the procedures of the WENO-JS [17], WENO-M [15], WENO-PM6 [7] and WENO-IM $(k, A)$ [8] schemes are reviewed in Section 2. In Section 3, we present a detailed analysis on how the nonlinear weights are mapped in some existing mapped WENO schemes and the consequences of these mappings on the numerical solutions. In Section 4, we propose a set of mapping functions that is order-preserving, as well as its properties, and apply it to construct the new mapped WENO scheme. Numerical experiments are presented in Section 5 to illustrate the advantages of the proposed WENO scheme. Finally, some concluding remarks are made in Section 6 to close this paper.

\section{Brief review of the fifth-order WENO schemes}

\subsection{Finite volume methodology}

We consider the finite volume method for the following one-dimensional scalar hyperbolic conservation laws

$$
\left\{\begin{array}{l}
\frac{\partial u}{\partial t}+\frac{\partial f(u)}{\partial x}=0, \quad x_{l}<x<x_{r}, t>0 \\
u(x, 0)=u_{0}(x) .
\end{array}\right.
$$

For brevity in the description, we assume that the computational domain is discretized into uniform cells $I_{j}=$ $\left[x_{j-1 / 2}, x_{j+1 / 2}\right], j=1, \cdots, N$ with the uniform cell size $\Delta x=\frac{x_{r}-x_{l}}{N}$, and the associated cell centers and cell boundaries are denoted by $x_{j}=x_{l}+(j-1 / 2) \Delta x$ and $x_{j \pm 1 / 2}=x_{j} \pm \Delta x / 2$ respectively. Let $\bar{u}\left(x_{j}, t\right)=\frac{1}{\Delta x} \int_{x_{j-1 / 2}}^{x_{j+1 / 2}} u(\xi, t) \mathrm{d} \xi$ be the cell average of $I_{j}$, then by integrating Eq.2 2 over the control volumes $I_{j}$ and employing some simple mathematical manipulations, we approximate Eq.2) by the following finite volume conservative formulation

$$
\frac{\mathrm{d} \bar{u}_{j}(t)}{\mathrm{d} t} \approx-\frac{1}{\Delta x}\left(\hat{f}\left(u_{j+1 / 2}^{-}, u_{j+1 / 2}^{+}\right)-\hat{f}\left(u_{j-1 / 2}^{-}, u_{j-1 / 2}^{+}\right)\right)
$$

where $\bar{u}_{j}(t)$ is the numerical approximation to the cell average $\bar{u}\left(x_{j}, t\right)$, and the numerical flux $\hat{f}\left(u_{j \pm 1 / 2}^{-}, u_{j \pm 1 / 2}^{+}\right)$where $u_{j \pm 1 / 2}^{ \pm}$refer to the limits of $u$ is a replacement of the physical flux function $f(u)$ at the cell boundaries $x_{j \pm 1 / 2}$. The values of $u_{j \pm 1 / 2}^{ \pm}$can be obtained by the technique of reconstruction like some WENO reconstructions narrated later. In this paper, we choose the global Lax-Friedrichs flux $\hat{f}(a, b)=\frac{1}{2}[f(a)+f(b)-\alpha(b-a)]$, where $\alpha=\max _{u}\left|f^{\prime}(u)\right|$ is a constant and the maximum is taken over the whole range of $u$. For the hyperbolic conservation laws system, a local characteristic decomposition is commonly used and more details can be found in [17]. In two-dimensional Cartesian meshes, two classes of finite volume WENO schemes are studied in detail in [35], and we take the one denoted as class $\mathrm{A}$ in this paper.

The ordinary differential equation (ODE) system Eq. (3) can be solved using a suitable time discretization, and the following explicit, third-order, strong stability preserving (SSP) Runge-Kutta method [30, 9, 10] is employed in our calculations

$$
\begin{aligned}
& u^{(1)}=u^{n}+\Delta t \mathcal{L}\left(u^{n}\right), \\
& u^{(2)}=\frac{3}{4} u^{n}+\frac{1}{4} u^{(1)}+\frac{1}{4} \Delta t \mathcal{L}\left(u^{(1)}\right), \\
& u^{n+1}=\frac{1}{3} u^{n}+\frac{2}{3} \Delta t \mathcal{L}\left(u^{(2)}\right),
\end{aligned}
$$

where

$$
\mathcal{L}\left(u_{j}\right):=-\frac{1}{\Delta x}\left(\hat{f}\left(u_{j+1 / 2}^{-}, u_{j+1 / 2}^{+}\right)-\hat{f}\left(u_{j-1 / 2}^{-}, u_{j-1 / 2}^{+}\right)\right),
$$

and $u^{(1)}, u^{(2)}$ are the intermediate stages, $u^{n}$ is the value of $u$ at time level $t^{n}=n \Delta t$, and $\Delta t$ is the time step satisfying some proper CFL condition. As mentioned earlier, the WENO reconstructions will be applied to obtain $\mathcal{L}\left(u_{j}\right)$. 


\subsection{WENO-JS}

We review the process of the fifth-order WENO-JS reconstruction [17]. As the right-biased reconstruction $u_{j+1 / 2}^{+}$ can easily be obtained by mirror symmetry with respect to the location $x_{j+1 / 2}$ of that for the left-biased one $u_{j+1 / 2}^{-}$, we describe only the reconstruction procedure of $u_{j+1 / 2}^{-}$. For simplicity of notation, we do not use the subscript " $-"$ in the following content.

For constructing the values of $u_{j+1 / 2}$ from known cell average values, the fifth-order WENO-JS scheme uses a 5-point global stencil $S^{5}=\left\{I_{j-2}, I_{j-1}, I_{j}, I_{j+1}, I_{j+2}\right\}$. Normally, $S^{5}$ is subdivided into three 3-point substencils $S_{s}=\left\{I_{j+s-2}, I_{j+s-1}, I_{j+s}\right\}, s=0,1,2$. Explicitly, the third-order approximations of $u\left(x_{j+1 / 2}, t\right)$ associated with these substencils are given by

$$
\begin{aligned}
& u_{j+1 / 2}^{0}=\frac{1}{6}\left(2 \bar{u}_{j-2}-7 \bar{u}_{j-1}+11 \bar{u}_{j}\right), \\
& u_{j+1 / 2}^{1}=\frac{1}{6}\left(-\bar{u}_{j-1}+5 \bar{u}_{j}+2 \bar{u}_{j+1}\right), \\
& u_{j+1 / 2}^{2}=\frac{1}{6}\left(2 \bar{u}_{j}+5 \bar{u}_{j+1}-2 \bar{u}_{j+2}\right) .
\end{aligned}
$$

The above three third-order approximations are combined in a weighted average to define the fifth-order WENO approximation of $u\left(x_{j+1 / 2}, t\right)$,

$$
u_{j+1 / 2}=\sum_{s=0}^{2} \omega_{s} u_{j+1 / 2}^{s},
$$

where $\omega_{s}$ is the nonlinear weight of the substencil $S_{s}$. In the classic WENO-JS scheme, $\omega_{s}$ is calculated as

$$
\omega_{s}^{\mathrm{JS}}=\frac{\alpha_{s}^{\mathrm{JS}}}{\sum_{l=0}^{2} \alpha_{l}^{\mathrm{JS}}}, \alpha_{s}^{\mathrm{JS}}=\frac{d_{s}}{\left(\epsilon+\beta_{s}\right)^{2}}, \quad s=0,1,2,
$$

where $d_{0}=0.1, d_{1}=0.6, d_{2}=0.3$ are ideal weights of $\omega_{s}$ satisfying $\sum_{s=0}^{2} d_{s} u_{j+1 / 2}^{s}=u\left(x_{j+1 / 2}, t\right)+O\left(\Delta x^{5}\right)$ in smooth regions, $\epsilon$ is a small positive number introduced to prevent the denominator from becoming zero, and the parameters $\beta_{s}$ are the smoothness indicators for the third-order approximations $u_{j+1 / 2}^{s}$ and their explicit forms defined by Jiang and Shu [17] is given as

$$
\begin{aligned}
& \beta_{0}=\frac{13}{12}\left(\bar{u}_{j-2}-2 \bar{u}_{j-1}+\bar{u}_{j}\right)^{2}+\frac{1}{4}\left(\bar{u}_{j-2}-4 \bar{u}_{j-1}+3 \bar{u}_{j}\right)^{2}, \\
& \beta_{1}=\frac{13}{12}\left(\bar{u}_{j-1}-2 \bar{u}_{j}+\bar{u}_{j+1}\right)^{2}+\frac{1}{4}\left(\bar{u}_{j-1}-\bar{u}_{j+1}\right)^{2}, \\
& \beta_{2}=\frac{13}{12}\left(\bar{u}_{j}-2 \bar{u}_{j+1}+\bar{u}_{j+2}\right)^{2}+\frac{1}{4}\left(3 \bar{u}_{j}-4 \bar{u}_{j+1}+\bar{u}_{j+2}\right)^{2} .
\end{aligned}
$$

The fifth-order WENO-JS scheme is able to achieve optimal order of accuracy in smooth regions without critical points. However, it loses accuracy and its order of accuracy decreases to third-order or even less at critical points. More details can be found in [15].

\subsection{WENO-M}

It has been indicated that [15, 2, 8, 26, 6] a sufficient condition that ensures the fifth-order WENO schemes retaining optimal order of convergence is simply given by

$$
\omega_{s}^{ \pm}-d_{s}=O\left(\Delta x^{3}\right), \quad s=0,1,2 .
$$

The condition Eq. (7) may not hold in the case of smooth extrema or at critical points when the fifth-order WENO-JS scheme is used. Henrick et al. [15] proposed a fix to this deficiency in their WENO-M scheme by introducing a mapping function that makes $\omega_{s}$ approximating the ideal weights $d_{s}$ with increased accuracy. The mapping function of the nonlinear weights $\omega \in[0,1]$ is given by

$$
\left(g^{\mathrm{M}}\right)_{s}(\omega)=\frac{\omega\left(d_{s}+d_{s}^{2}-3 d_{s} \omega+\omega^{2}\right)}{d_{s}^{2}+\left(1-2 d_{s}\right) \omega}, \quad s=0,1,2 .
$$


One can verify that $\left(g^{\mathrm{M}}\right)_{s}(\omega)$ meets the requirement in Eq. 7 , and clearly, this mapping function is a non-decreasing monotone function on $[0,1]$ with finite slopes which satisfies the following properties.

Lemma 1. The mapping function $\left(g^{\mathrm{M}}\right)_{s}(\omega)$ defined by Eq. (8) satisfies:

$C 1.0 \leq\left(g^{\mathrm{M}}\right)_{s}(\omega) \leq 1,\left(g^{\mathrm{M}}\right)_{s}(0)=0,\left(g^{\mathrm{M}}\right)_{s}(1)=1$;

C2. $\left(g^{\mathrm{M}}\right)_{s}\left(d_{s}\right)=d_{s}$;

C3. $\left(g^{\mathrm{M}}\right)_{s}^{\prime}\left(d_{s}\right)=\left(g^{\mathrm{M}}\right)_{s}^{\prime \prime}\left(d_{s}\right)=0$.

With the mapping function defined by Eq. 87, the nonlinear weights of the WENO-M scheme are defined as

$$
\omega_{s}^{\mathrm{M}}=\frac{\alpha_{s}^{\mathrm{M}}}{\sum_{l=0}^{2} \alpha_{l}^{\mathrm{M}}}, \alpha_{s}^{\mathrm{M}}=\left(g^{\mathrm{M}}\right)_{s}\left(\omega_{s}^{\mathrm{JS}}\right), \quad s=0,1,2,
$$

where $\omega_{s}^{\mathrm{JS}}$ are calculated by Eq. $(6)$.

In [15], it has been analyzed and proved in detail that the WENO-M scheme can retain the optimal order of accuracy in smooth regions even near the first-order critical points.

\subsection{WENO-PMk}

Recently, Feng et al. [7] noticed that the mapping function $\left(g^{\mathrm{M}}\right)_{s}(\omega)$ in Eq. 8) amplifies the effect from the nonsmooth stencils by a factor of $\left(1+1 / d_{s}\right)$ as its first derivative satisfies $\left(g^{\mathrm{M}}\right)_{s}^{\prime}(0)=1+1 / d_{s}$. They argued that this may cause the potential loss of accuracy near the discontinuities or the parts with sharp gradients. To address this issue, Feng et al. [7] add two requirements, that is, $g_{s}^{\prime}(0)=0$ and $g_{s}^{\prime}(1)=0$, to the original criteria as shown in Lemma 1 . To meet these criteria they proposed a new mapping by the following piecewise polynomial function

$$
\left(g^{\mathrm{PM}}\right)_{s}(\omega)=c_{1}\left(\omega-d_{s}\right)^{k+1}\left(\omega+c_{2}\right)+d_{s}, \quad k \geq 2, s=0,1,2,
$$

where $c_{1}, c_{2}$ are constants with specified parameters $k$ and $d_{s}$, taking the following forms

$$
c_{1}=\left\{\begin{array}{ll}
(-1)^{k} \frac{k+1}{d_{s}^{k+1}}, & 0 \leq \omega \leq d_{s}, \\
-\frac{k+1}{\left(1-d_{s}\right)^{k+1}}, & d_{s}<\omega \leq 1,
\end{array} \quad c_{2}= \begin{cases}\frac{d_{s}}{k+1}, & 0 \leq \omega \leq d_{s}, \\
\frac{d_{s}-(k+2)}{k+1}, & d_{s}<\omega \leq 1 .\end{cases}\right.
$$

Lemma 2. The mapping function $\left(g^{\mathrm{PM}}\right)_{s}(\omega)$ defined by Eq. (9) satisfies:

Cl. $\left(g^{\mathrm{PM}}\right)_{s}^{\prime}(\omega) \geq 0, \omega \in[0,1]$;

$C 2 .\left(g^{\mathrm{PM}}\right)_{s}(0)=0,\left(g^{\mathrm{PM}}\right)_{s}(1)=1,\left(g^{\mathrm{PM}}\right)_{s}\left(d_{s}\right)=d_{s}$;

C3. $\left(g^{\mathrm{PM}}\right)_{s}^{\prime}\left(d_{s}\right)=\cdots=\left(g^{\mathrm{PM}}\right)_{s}^{(k)}\left(d_{s}\right)=0$;

C4. $\left(g^{\mathrm{PM}}\right)_{s}^{\prime}(0)=\left(g^{\mathrm{PM}}\right)_{s}^{\prime}(1)=0$.

Similarly, with the mapping function defined by Eq.9 where the parameter $k$ is taken to be 6 as recommended in [7], the WENO-PM6 scheme is proposed by computing the nonlinear weights as

$$
\omega_{s}^{\mathrm{PM} 6}=\frac{\alpha_{s}^{\mathrm{PM} 6}}{\sum_{l=0}^{2} \alpha_{l}^{\mathrm{PM} 6}}, \alpha_{s}^{\mathrm{PM} 6}=\left(g^{\mathrm{PM} 6}\right)_{s}\left(\omega_{s}^{\mathrm{JS}}\right), \quad s=0,1,2 .
$$

It has been shown by numerical experiments [7, 34] that the two additional requirements are effective and the resolution near discontinuities of the WENO-PM6 scheme is significantly higher than those of the WENO-JS and WENO-M schemes, especially for long output times. We refer to [7] for more details. 


\section{5. $W E N O-I M(k, A)$}

Feng et al. [8] has proposed the WENO-IM $(k, A)$ scheme by rewriting the mapping function of the WENO-M scheme as shown in Eq. 88. The broader class of improved mapping functions $\left(g^{\mathrm{IM}}\right)_{s}(\omega ; k, A)$ is defined by

$$
\left(g^{\mathrm{IM}}\right)_{s}(\omega ; k, A)=d_{s}+\frac{\left(\omega-d_{s}\right)^{k+1} A}{\left(\omega-d_{s}\right)^{k} A+\omega(1-\omega)}, \quad A>0, k=2 n, n \in \mathbb{N}^{+}, s=0,1,2 .
$$

Then, the associated nonlinear weights are given by

$$
\omega_{s}^{\mathrm{IM}}=\frac{\alpha_{s}^{\mathrm{IM}}}{\sum_{l=0}^{2} \alpha_{l}^{\mathrm{IM}}}, \alpha_{s}^{\mathrm{IM}}=\left(g^{\mathrm{IM}}\right)_{s}\left(\omega_{s}^{\mathrm{JS}} ; k, A\right), \quad s=0,1,2 .
$$

It is trivial to show that $\left(g^{\mathrm{M}}\right)_{s}(\omega)$ belongs to the $\left(g^{\mathrm{IM}}\right)_{s}(\omega ; k, A)$ family of functions as $\left(g^{\mathrm{M}}\right)_{s}(\omega)=\left(g^{\mathrm{IM}}\right)_{s}(\omega ; 2,1)$. Actually, the selection of parameters $k$ and $A$ has been discussed carefully in [8], and $k=2, A=0.1$ was recommended.

Lemma 3. The mapping function $\left(g^{\mathrm{IM}}\right)_{s}(\omega ; k, A)$ defined by Eq. 10p satisfies:

$$
\begin{aligned}
& \text { C1. }\left(g^{\mathrm{IM}}\right)_{s}^{\prime}(\omega ; k, A) \geq 0, \omega \in[0,1] ; \\
& \text { C2. }\left(g^{\mathrm{IM}}\right)_{s}(0 ; k, A)=0,\left(g^{\mathrm{IM}}\right)_{s}(1 ; k, A)=1 ; \\
& \text { C2. }\left(g^{\mathrm{IM}}\right)_{s}\left(d_{s} ; k, A\right)=d_{s} ; \\
& \text { C3. }\left(g^{\mathrm{IM}}\right)_{s}^{\prime}\left(d_{s} ; k, A\right)=\cdots=\left(g^{\mathrm{IM}}\right)_{s}^{(k)}\left(d_{s} ; k, A\right)=0,\left(g^{\mathrm{IM}}\right)_{s}^{(k+1)}\left(d_{s} ; k, A\right) \neq 0 .
\end{aligned}
$$

We refer to $[8]$ for the detailed proof of Lemma 3

\section{Analysis of the nonlinear weights of the existing mapped WENO schemes}

\subsection{Monotone increasing piecewise mapping function and the generalized WENO-ACM schemes}

In [20], the present authors have proposed the fifth-order WENO-ACM scheme. It has been demonstrated that taking narrower transition intervals (standing for the intervals over which the mapping results are in a transition from 0 to $d_{s}$ or from $d_{s}$ to 1 ) of the mapping function does not bring any adverse effects on the resolutions and convergence orders, and the associated scheme still performs very well even if the transition intervals are infinitely close to 0 . Therefore, we can set the transition intervals to be 0 leading to a simpler form of the mapping function as follows

$$
\left(g^{\mathrm{MIP}-\mathrm{ACM}}\right)_{s}(\omega)= \begin{cases}0, & \omega \in \Omega_{1}=\left[0, \mathrm{CFS}_{s}\right), \\ d_{s}, & \omega \in \Omega_{2}=\left[\mathrm{CFS}_{s}, \overline{\mathrm{CFS}}_{s}\right], \\ 1, & \omega \in \Omega_{3}=\left(\overline{\mathrm{CFS}}_{s}, 1\right],\end{cases}
$$

where $\mathrm{CFS}_{s}$ is the same as that in [19, 20] satisfying $\mathrm{CFS}_{s} \in\left(0, d_{s}\right)$, and $\overline{\mathrm{CFS}}_{s}=1-\frac{1-d_{s}}{d_{s}} \times \mathrm{CFS}_{s}$ with $\overline{\mathrm{CFS}}_{s} \in\left(d_{s}, 1\right)$. Clearly, $\left(g^{\mathrm{MIP}-\mathrm{ACM}}\right)_{s}(\omega)$ is a discontinuous function with two jump discontinuities at $\omega=\mathrm{CFS}_{s}$ and $\omega=\overline{\mathrm{CFS}}_{s}$ on the interval $[0,1]$, while differentiable mapping functions on the interval $[0,1]$ were required in previously published mapped WENO schemes [15, 7, 8, 34, 21, 32, 33, 19, 20]. However, after extensive numerical tests, we find that a continuous mapping function is not essential in the design of the mapped WENO scheme. Actually, in the evaluation at $\omega_{s}^{\mathrm{JS}}$ of the Taylor series approximations of the mapping function about the optimal weights $d_{s}$, which plays the core role in the convergence analysis of the mapped WENO schemes (originally proposed by Henrick in the statement of page 556 in [15]), one needs only the mapping function to be differentiable near the neighborhood of $\omega=d_{s}$ but not over the whole range of $\omega \in[0,1]$. Therefore, we innovatively propose the definition of the monotone increasing piecewise mapping function.

Definition 1. (monotone increasing piecewise mapping function) Let $\Omega=[0,1]$, and assume that $\Omega$ is divided into a sequence of nonoverlapping intervals $\Omega_{i}, i=1,2, \cdots, M$, that is, $\Omega=\Omega_{1} \cup \Omega_{2} \cup \cdots \cup \Omega_{M}$ and $\Omega_{i} \cap \Omega_{j}=\varnothing$, for $\forall i, j=1,2, \cdots, M$ and $i \neq j$. Let $\bar{\Omega}_{i}=\left\{\omega \mid \omega \in \Omega_{i}\right.$ and $\left.\omega \notin \partial \Omega_{i}\right\}$, and suppose that $\left(g^{\mathrm{MIP}-\mathrm{X}}\right)_{s}(\omega)$ is a mapping function on the interval $[0,1]$, then $\left(g^{\mathrm{MIP}-\mathrm{X}}\right)_{s}(\omega)$ is called a monotone increasing piecewise mapping function, if it satisfies the following conditions: (C1) for $\forall \omega \in \bar{\Omega}_{i}, i=1, \cdots, M,\left(g^{\mathrm{MIP}-\mathrm{X}}\right)_{s}(\omega)$ is differentiable and $\left(g^{\mathrm{MIP}-\mathrm{X}}\right)_{s}^{\prime}(\omega) \geq 0 ;(C 2)$ for $\forall \omega_{i}, \omega_{j} \in \Omega$, if $\omega_{i} \geq \omega_{j}$, then $\left(g^{\mathrm{MIP}-\mathrm{X}}\right)_{s}\left(\omega_{i}\right) \geq\left(g^{\mathrm{MIP}-\mathrm{X}}\right)_{s}\left(\omega_{j}\right)$. 
It is trivial to verify that $\left(g^{\mathrm{MIP}-\mathrm{ACM}}\right)_{s}(\omega)$ defined by Eq. $[11)$ is a monotone increasing piecewise mapping function and it satisfies the following properties.

Lemma 4. The mapping function $\left(g^{\mathrm{MIP}-\mathrm{ACM}}\right)_{s}(\omega)$ defined by Eq. $(11)$ satisfies the following properties:

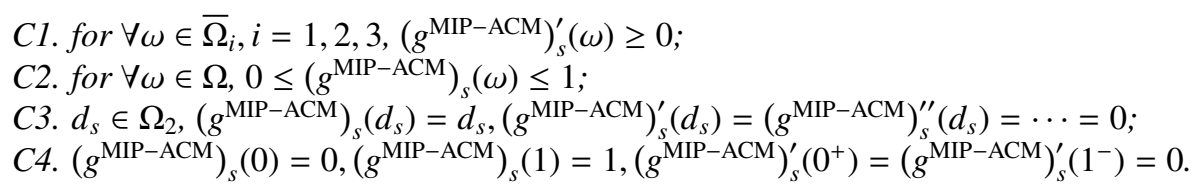

Naturally, we can derive a generalized version of the mapping function $\left(g^{\mathrm{MIP}-\mathrm{ACM}}\right)_{s}(\omega)$ as follows

$$
\left(g^{\mathrm{MIP}-\mathrm{ACM} k}\right)_{s}(\omega)= \begin{cases}k_{s} \omega, & \omega \in \Omega_{1}, \\ d_{s}, & \omega \in \Omega_{2}, \\ 1-k_{s}(1-\omega), & \omega \in \Omega_{3},\end{cases}
$$

where $\Omega_{1}, \Omega_{2}, \Omega_{3}$ are the same as in Eq. 11 and $k_{s} \in\left[0, \frac{d_{s}}{\mathrm{CFS}_{s}}\right]$. Obviously, if $k_{s}$ is taken to be $0,\left(g^{\mathrm{MIP}-\mathrm{ACM} k}\right)_{s}(\omega)$ exactly turns into $\left(g^{\mathrm{MIP}-\mathrm{ACM}}\right)_{s}(\omega)$. Thus, we need only discuss $\left(g^{\mathrm{MIP}-\mathrm{ACM} k}\right)_{s}(\omega)$. Similarly, it is easy to know that $\left(g^{\mathrm{MIP}-\mathrm{ACM} k}\right)_{s}(\omega)$ is a monotone increasing piecewise mapping function and it satisfies the following properties.

Lemma 5. The mapping function $\left(g^{\mathrm{MIP}-\mathrm{ACM} k}\right)_{s}(\omega)$ defined by Eq. 12 , satisfies the following properties:

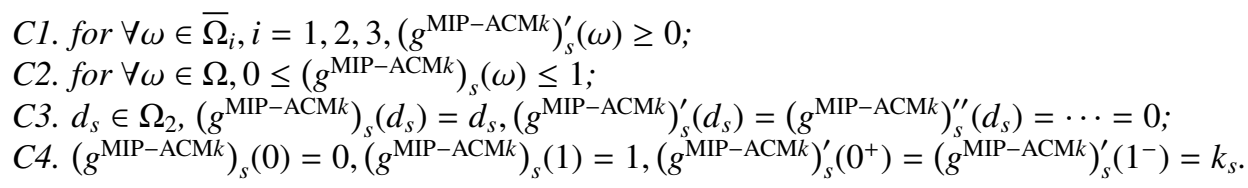

As the proofs of Lemma 4 and Lemma 5 are very easy, we do not state them here and we can observe these properties intuitively from the $\left(g^{\mathrm{MIP}-\mathrm{ACM} k}\right)_{s}(\omega) \sim \omega$ curves as shown in Fig. 3 below.

Now, we give the monotone increasing piecewise approximate-constant-mapped WENO scheme, denoted as MIPWENO-ACM $k$, with the mapped weights as follows

$$
\omega_{s}^{\mathrm{MIP}-\mathrm{ACM} k}=\frac{\alpha_{s}^{\mathrm{MIP}-\mathrm{ACM} k}}{\sum_{l=0}^{2} \alpha_{l}^{\mathrm{MIP}-\mathrm{ACM} k}}, \alpha_{s}^{\mathrm{MIP}-\mathrm{ACM} k}=\left(g^{\mathrm{MIP}-\mathrm{ACM} k}\right)_{s}\left(\omega_{s}^{\mathrm{JS}}\right) .
$$

We present Theorem 2, which will show that the MIP-WENO-ACM $k$ scheme can recover the optimal convergence orders for different values of $n_{\mathrm{cp}}$ in smooth regions.

Theorem 1. When $\mathrm{CFS}_{s} \ll d_{s}$, for $\forall n_{\mathrm{cp}}<r-1$, the $(2 r-1)$ th-order MIP-WENO-ACMk scheme can achieve the optimal convergence rates of accuracy if the new mapping function $\left(g^{\mathrm{MIP}-\mathrm{ACM} k}\right)_{s}(\omega)$ is applied to the weights of the $(2 r-1)$ th-order WENO-JS scheme.

We can prove Theorem 1 by employing the Taylor series analysis and using Lemma 5 of this paper and Lemma 1 and Lemma 2 in the statement of page 456 to 457 in [8], and the detailed proof process is almost identical to the one in [15].

\subsection{Discussion about the effects of $g^{\prime}(0)$ of the mapped WENO schemes on resolutions and spurious oscillations}

In order to study the effects of $g^{\prime}(0)$ of the mapped WENO schemes on resolutions and spurious oscillations in simulating the problems with discontinuities, we calculate the one-dimensional linear advection equation

$$
u_{t}+u_{x}=0
$$


with the following initial condition [17]

$$
u(x, 0)= \begin{cases}\frac{1}{6}[G(x, \beta, z-\hat{\delta})+4 G(x, \beta, z)+G(x, \beta, z+\hat{\delta})], & x \in[-0.8,-0.6], \\ 1, & x \in[-0.4,-0.2], \\ 1-|10(x-0.1)|, & x \in[0.0,0.2], \\ \frac{1}{6}[F(x, \alpha, a-\hat{\delta})+4 F(x, \alpha, a)+F(x, \alpha, a+\hat{\delta})], & x \in[0.4,0.6], \\ 0, & \text { otherwise, }\end{cases}
$$

where $G(x, \beta, z)=\mathrm{e}^{-\beta(x-z)^{2}}, F(x, \alpha, a)=\sqrt{\max \left(1-\alpha^{2}(x-a)^{2}, 0\right)}$, and the constants are $z=-0.7, \hat{\delta}=0.005, \beta=$ $\frac{\log 2}{36 \hat{\delta}^{2}}, a=0.5$ and $\alpha=10$. The periodic boundary condition is used in the two directions and the CFL number is set to be 0.1 . This problem consists of a Gaussian, a square wave, a sharp triangle and a semi-ellipse. For brevity in the presentation, we call this Linear Problem SLP as it is presented by Shu et al. in [17].

The following two groups of mapped WENO schemes with various values of $\left(g^{X}\right)^{\prime}(0)$ (X stands for some specific mapped WENO scheme) are employed in the discussion.

\subsubsection{Study on the WENO-PM6 and WENO-IM( $k, A)$ schemes}

In this subsection, we focus on the performances of the WENO-PM6 scheme [7] and the WENO-IM( $k, A) \operatorname{schemes}$ [8] with $k=2$ and $A=0.1,0.5$ on solving SLP. A uniform mesh size of $N=400$ is used and the output time is set to be $t=200$.

From Fig. 11 we can easily see that the values of $\left(g^{\mathrm{X}}\right)^{\prime}(0)$ satisfy

$$
\left(g^{\mathrm{PM} 6}\right)^{\prime}(0)<\left(g^{\mathrm{IM}(2,0.5)}\right)^{\prime}(0)<\left(g^{\mathrm{IM}(2,0.1)}\right)^{\prime}(0) .
$$

Fig. 2 shows the calculating results, and Table 1 shows the $L_{1}, L_{2}, L_{\infty}$ errors and the order of these errors (in brackets in descending manner), i.e., in the second column, 1 indicates the largest $L_{1}$ error and 2 indicates the second largest one, etc. From Fig. 2 and Table 1, we can observe that: (1) the WENO-IM(2,0.1) scheme, whose $\left(g^{\operatorname{IM}(2,0.1)}\right)^{\prime}(0)$ is the largest, presents the smallest spurious oscillation (actually, there is no spurious oscillation in present computing conditions) and gives the smallest $L_{1}, L_{2}$ and $L_{\infty}$ errors; (2) the WENO-PM6 scheme, whose $\left(g^{\mathrm{PM} 6}\right)^{\prime}(0)$ is the smallest and satisfies $\left(g^{\mathrm{PM} 6}\right)^{\prime}(0)=0$, presents the largest spurious oscillation and gives the second largest $L_{1}, L_{2}$ and $L_{\infty}$ errors; (3) the WENO-IM $(2,0.5)$ scheme shows the lowest resolutions and presents evident spurious oscillation at the top of the square wave, and it gives the largest $L_{1}, L_{2}$ and $L_{\infty}$ errors, while its $\left(g^{\mathrm{IM}(2,0.5)}\right)^{\prime}(0)$ is neither largest nor smallest.
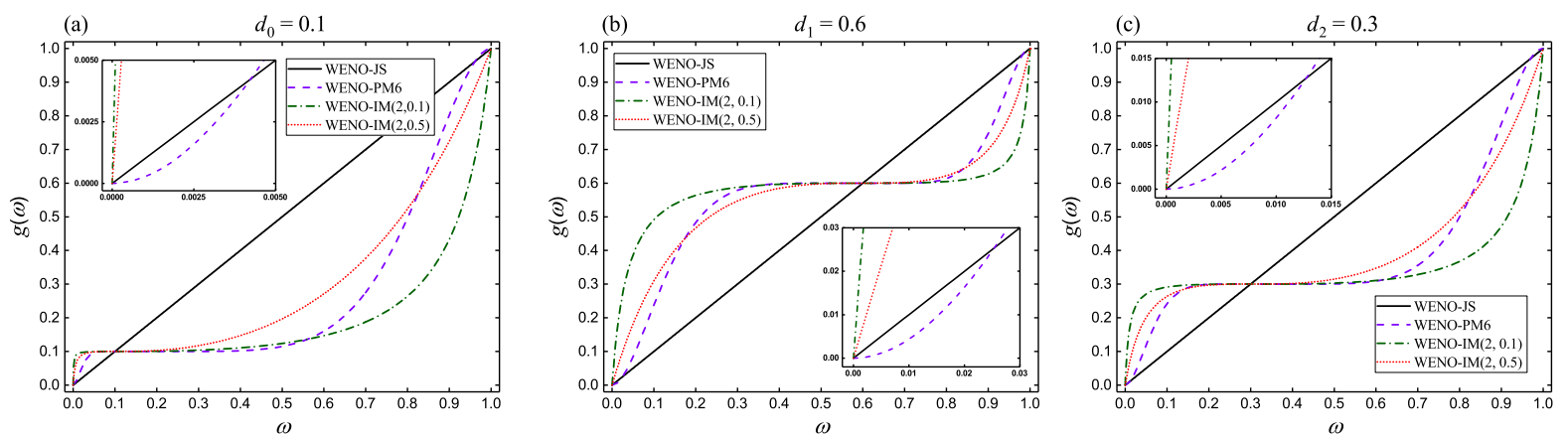

Fig. 1. The mapping functions of the WENO-PM6, WENO-IM $(2,0.1)$ and WENO-IM $(2,0.5) \operatorname{schemes}, d_{0}=0.1, d_{1}=$ $0.6, d_{2}=0.3$.

\subsubsection{Study on the WENO-MAIM2 and MIP-WENO-ACMk schemes}

In this subsection, we focus on the performances of the WENO-MAIM2 [19] and MIP-WENO-ACM $k$ schemes on solving SLP. We still use a uniform mesh size of $N=400$ and choose the output time $t=200$. 


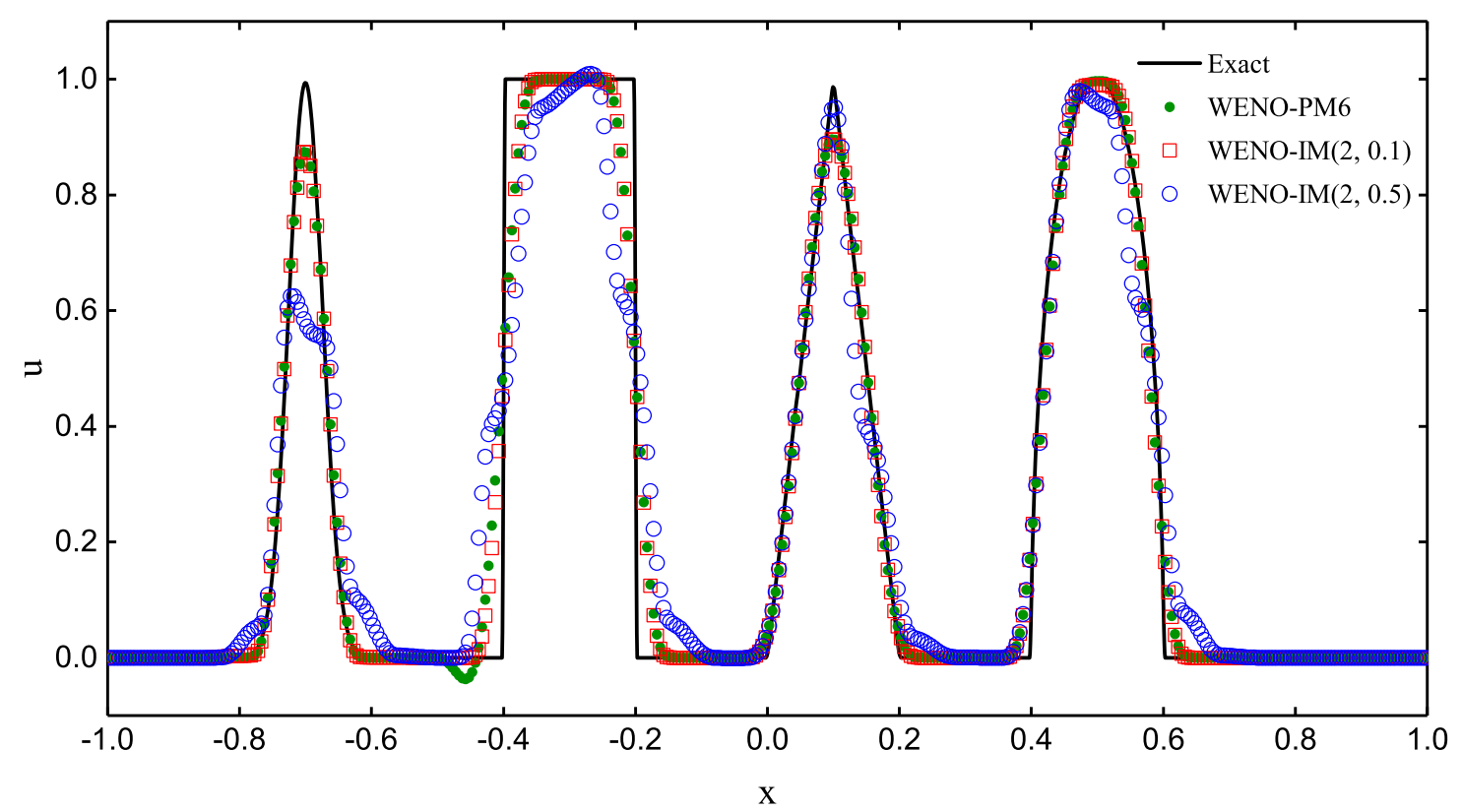

Fig. 2. Performance of the fifth-order WENO-PM6, WENO-IM $(2,0.1)$ and WENO-IM(2, 0.5) schemes for SLP with $N=400$ at long output time $t=200$.

Table 1. The $L_{1}, L_{2}, L_{\infty}$ errors for SLP with $N=400$ at long output time $t=200$, computed by the WENO-PM6, WENO-IM(2,0.1) and WENO-IM(2,0.5) schemes.

\begin{tabular}{llll}
\hline Schemes & $L_{1}$ error & $L_{2}$ error & $L_{\infty}$ error \\
\hline WENO-PM6 & $5.69929 \mathrm{e}-02(2)$ & $1.06646 \mathrm{e}-01(2)$ & $4.80453 \mathrm{e}-01(2)$ \\
WENO-IM(2, 0.1) & $5.46038 \mathrm{e}-02(3)$ & $1.04363 \mathrm{e}-01(3)$ & $4.52252 \mathrm{e}-01(3)$ \\
WENO-IM $(2,0.5)$ & $1.23950 \mathrm{e}-01(1)$ & $1.75554 \mathrm{e}-01(1)$ & $5.24783 \mathrm{e}-01(1)$ \\
\hline
\end{tabular}

As shown in Table 2. 6 different test schemes (ts- $i, i=1, \cdots, 6)$ of the WENO-MAIM2 and MIP-WENO-ACM $k$ schemes with specified parameters leading to various values of $\left(g^{\mathrm{ts}-i}\right)^{\prime}(0)$ are used in the discussion. In Fig. 3, we plot the curves of $\left(g^{\text {ts }-i}\right)(\omega) \sim \omega, i=1, \cdots, 6$, and we can intuitively observe that the values of $\left(g^{\text {ts }-i}\right)^{\prime}(0)$ satisfy

$$
\left(g^{\mathrm{ts}-3}\right)^{\prime}(0)=\left(g^{\mathrm{ts}-5}\right)^{\prime}(0)<\left(g^{\mathrm{ts}-1}\right)^{\prime}(0)<\left(g^{\mathrm{ts}-4}\right)^{\prime}(0)<\left(g^{\mathrm{ts}-6}\right)^{\prime}(0)<\left(g^{\mathrm{ts}-2}\right)^{\prime}(0) .
$$

Fig. 4 shows the calculating results, and Table 3 shows the $L_{1}, L_{2}, L_{\infty}$ errors and the order of these errors (in brackets in descending manner). From Fig. 4 and Table 3, we can observe that: (1) all the 6 test schemes present spurious oscillations; (2) ts-2 shows more in number and bigger in size of the spurious oscillations than ts- 1 , and the $L_{1}, L_{2}, L_{\infty}$ errors of ts-2 are larger than those of ts-1; (3) however, although $\left(g^{\mathrm{ts}-4}\right)^{\prime}(0)>\left(g^{\mathrm{ts}-3}\right)^{\prime}(0)$ and $\left(g^{\mathrm{ts}-3}\right)^{\prime}(0)=0$, ts-4 shows fewer in number and smaller in size of the spurious oscillations than ts-3, and the $L_{1}, L_{2}, L_{\infty}$ errors of ts-4 are smaller than those of ts-3; (4) in addition, although $\left(g^{\text {ts }-5}\right)^{\prime}(0) \ll\left(g^{\text {ts }-6}\right)^{\prime}(0)$ and $\left(g^{\text {ts }-5}\right)^{\prime}(0)=0$, ts-5 shows comparable spurious oscillations both in number and in size with ts-6, and the $L_{1}, L_{2}, L_{\infty}$ errors of ts- 5 are very close to those of ts-6, or in other words, the $L_{1}$ error of ts- 5 is slightly smaller than that of ts-6, while the $L_{2}, L_{\infty}$ errors of ts-5 are slightly larger than those of ts-6.

\subsection{Analysis of the real-time mapping relationship}

\subsubsection{Definition of order-preserving/non-order-preserving mapping and important numerical experiments}

From the discussion above, we can conclude that it is not essential to prevent the corresponding mapped WENO scheme from generating spurious oscillations or causing potential loss of accuracy near discontinuities that the first 
Table 2. 6 different test schemes (ts-1, $\cdots, 6$ ) of the WENO-MAIM2 and MIP-WENO-ACM $k$ schemes with specified parameters.

\begin{tabular}{llll}
\hline ts-i & WENO-X & Parameters & $\left(g^{\mathrm{X}}\right)^{\prime}(0)$ \\
\hline ts-1 & WENO-MAIM2 & $k=10, A=1.0 \mathrm{e}-6, Q=1.0, \mathrm{CFS}_{s}=0.05$ & 1 \\
ts-2 & WENO-MAIM2 & $k=10, A=1.0 \mathrm{e}-6, Q=0.25, \mathrm{CFS}_{s}=0.05$ & $\gg 1$ \\
ts-3 & MIP-WENO-ACM $k$ & $k_{s}=0, \mathrm{CFS}_{s}=0.1 d_{s}$ & 0 \\
ts-4 & MIP-WENO-ACM $k$ & $k_{s}=10, \mathrm{CFS}_{s}=0.1 d_{s}$ & 10 \\
ts-5 & MIP-WENO-ACM $k$ & $k_{s}=0, \mathrm{CFS}_{s}=0.01 d_{s}$ & 0 \\
ts-6 & MIP-WENO-ACM $k$ & $k_{s}=100, \mathrm{CFS}_{s}=0.01 d_{s}$ & 100 \\
\hline
\end{tabular}
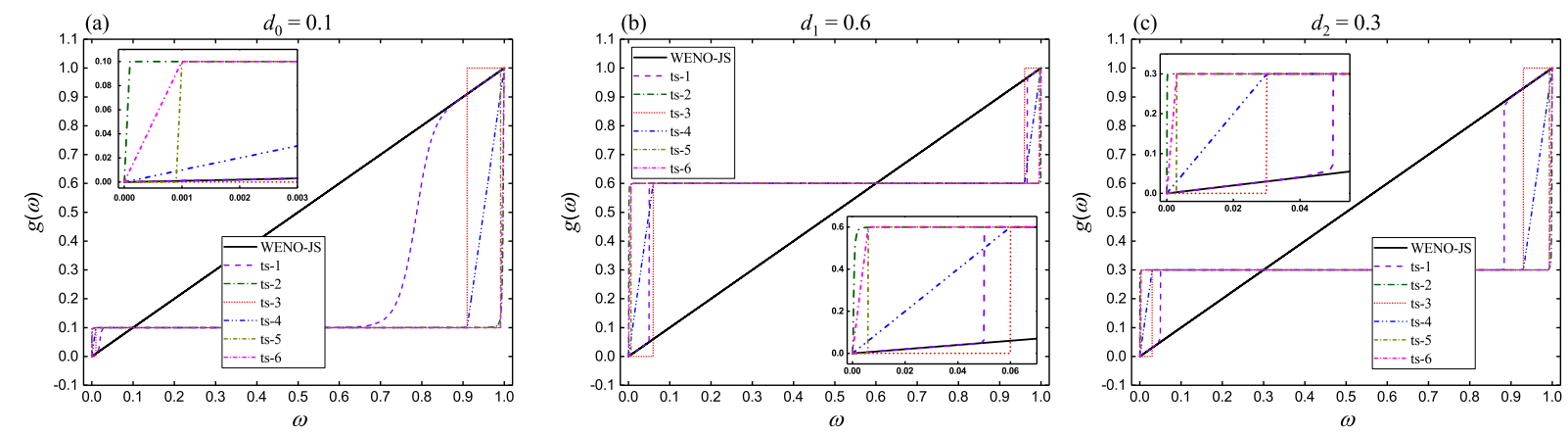

Fig. 3. The mapping functions of the test schemes shown in Table $2, d_{0}=0.1, d_{1}=0.6, d_{2}=0.3$.

derivatives of the mapping functions tend to 0 or a small value when $\omega$ is close to 0 . In this subsection, to discover the essential cause of the spurious oscillation generation and potential loss of accuracy, we will make a further analysis of the real-time mapping relationship $\left(g^{\mathrm{X}}\right)(\omega) \sim \omega$, that stands for the mapping relationship obtained from the calculation of some specific problem at specified output time but not directly obtained from the mapping function.

Before conducting the numerical experiments for the analysis, we propose the definition of order-preserving mapping and non-order-preserving mapping.

Definition 2. (order-preserving/non-order-preserving mapping) Suppose that $\left(g^{\mathrm{X}}\right)_{s}(\omega), s=0, \cdots, r-1$ is a monotone increasing piecewise mapping function of the $(2 r-1)$ th-order mapped WENO-X scheme. We say the set of mapping functions $\left\{\left(g^{\mathrm{X}}\right)_{s}(\omega), s=0, \cdots, r-1\right\}$ is order-preserving $(\boldsymbol{O P})$, iffor $\forall \omega_{a} \geq \omega_{b}$,

$$
\left(g^{\mathrm{X}}\right)_{m}\left(\omega_{a}\right) \geq\left(g^{\mathrm{X}}\right)_{n}\left(\omega_{b}\right), \quad \forall m, n \in\{0, \cdots, r-1\},
$$

where the equality holds if and only if $\omega_{a}=\omega_{b}$. Otherwise, we say the set of mapping functions $\left\{\left(g^{\mathrm{X}}\right)_{s}(\omega), s=\right.$ $0, \cdots, r-1\}$ is non-order-preserving (non-OP).

It is trivial to know that, even when the set of mapping functions $\left\{\left(g^{\mathrm{X}}\right)_{s}(\omega), s=0, \cdots, r-1\right\}$ is non-OP, Eq. 18 . may also hold at some points. Therefore, we add the following definition of OP point and non-OP point.

Definition 3. (OP point, non-OP point) Let $S^{2 r-1}$ denote the $(2 r-1)$-point global stencil centered around $x_{j}$. Assume that $S^{2 r-1}$ is subdivided into r-point substencils $\left\{S_{0}, \cdots, S_{r-1}\right\}$ and $\omega_{s}$ are the nonlinear weights corresponding to the substencils $S_{s}$ with $s=0, \cdots, r-1$, which are used as the independent variables by the mapping function. Suppose that $\left(g^{\mathrm{X}}\right)_{s}(\omega), s=0, \cdots, r-1$ is the mapping function of the mapped WENO-X scheme, then we say that a non-OP mapping process occurs at $x_{j}$, if $\exists m, n \in\{0, \cdots, r-1\}$, s.t.

$$
\begin{cases}\left(\omega_{m}-\omega_{n}\right)\left(\left(g^{\mathrm{X}}\right)_{m}\left(\omega_{m}\right)-\left(g^{\mathrm{X}}\right)_{n}\left(\omega_{n}\right)\right) \leq 0, & \text { if } \quad \omega_{m} \neq \omega_{n}, \\ \left(g^{\mathrm{X}}\right)_{m}\left(\omega_{m}\right) \neq\left(g^{\mathrm{X}}\right)_{n}\left(\omega_{n}\right), & \text { if } \quad \omega_{m}=\omega_{n} .\end{cases}
$$




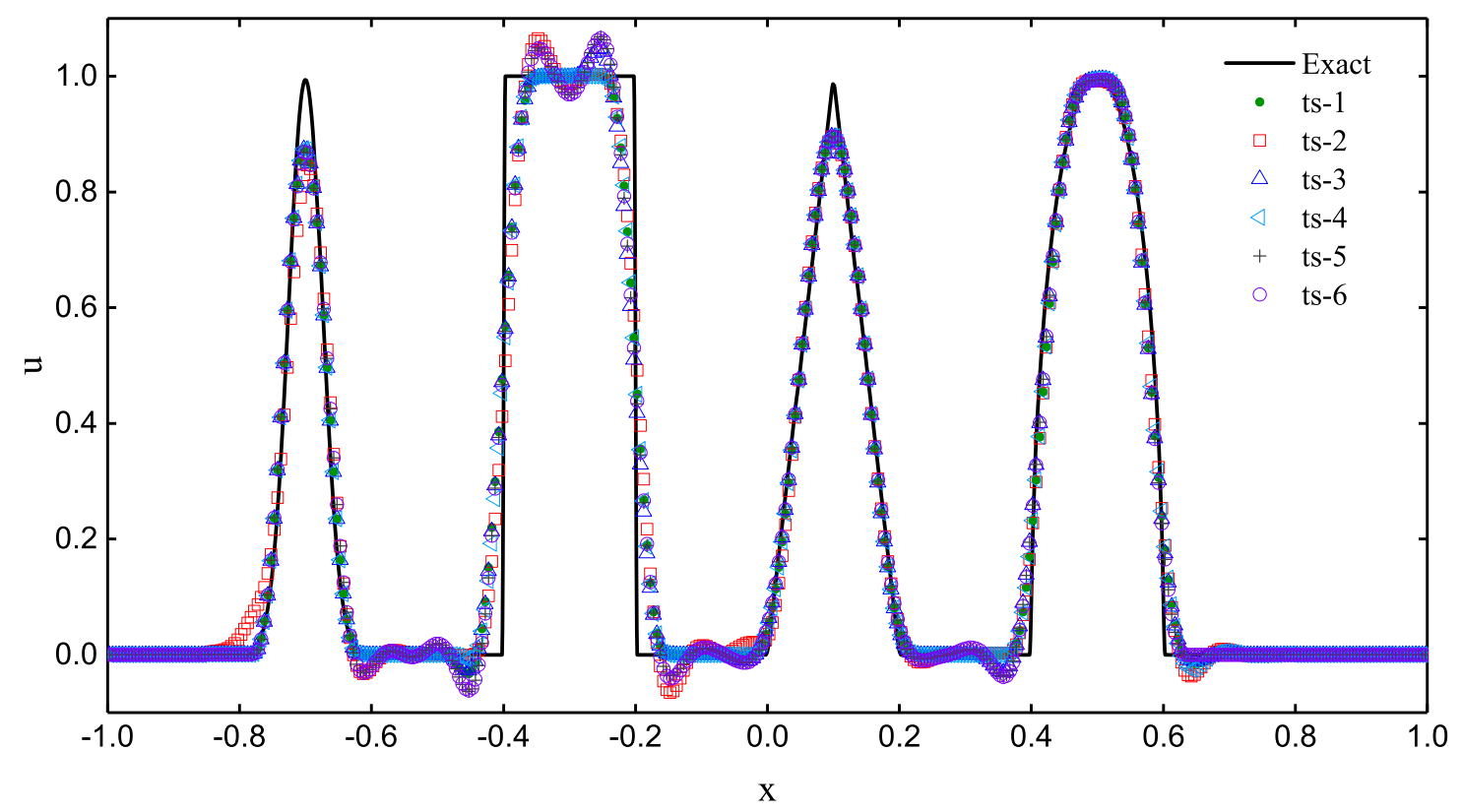

Fig. 4. Performance of the test schemes shown in Table 2 for the SLP with $N=400$ at long output time $t=200$.

Table 3. The $L_{1}, L_{2}, L_{\infty}$ errors for the SLP with $N=400$ at long output time $t=200$, computed by the test schemes shown in Table 2

\begin{tabular}{llll} 
Schemes, ts- $i$ & $L_{1}$ error & $L_{2}$ error & \\
\hline ts-1 & $5.71367 \mathrm{e}-02(5)$ & $1.06259 \mathrm{e}-01(5)$ & $L_{\infty}$ error \\
ts-2 & $6.80647 \mathrm{e}-02(1)$ & $1.09276 \mathrm{e}-01(1)$ & $4.76278 \mathrm{e}-01(3)$ \\
ts-3 & $5.91473 \mathrm{e}-02(4)$ & $1.07220 \mathrm{e}-01(4)$ & \\
ts-4 & $5.55635 \mathrm{e}-02(6)$ & $1.04530 \mathrm{e}-01(6)$ & $4.91997 \mathrm{e}-01(1)$ \\
ts-5 & $6.60760 \mathrm{e}-02(3)$ & $1.08378 \mathrm{e}-01(2)$ & $4.5954 \mathrm{e}-01(2)$ \\
ts-6 & $6.62020 \mathrm{e}-02(2)$ & $1.08289 \mathrm{e}-01(3)$ & \\
\hline
\end{tabular}

And we say $x_{j}$ is a non-OP point. Otherwise, we say $x_{j}$ is an OP point.

After extensive numerical experiments, we have discovered that, for almost all previously published mapped WENO schemes at least as far as we know, the non-OP mapping process will definitely occur when they are used for solving the problems with discontinuities. To demonstrate this, we still take the SLP as an example. The WENOPM6 [7], WENO-IM(2,0.1) [8] schemes and the MIP-WENO-ACM $k$ scheme with parameters $k_{s}=0, \mathrm{CFS}_{s}=\frac{d_{s}}{10}$ are used. A uniform mesh size of $N=400$ and two output times $t=2$ (short) and $t=200$ (long) are taken in all calculations. In Fig. 5 to Fig. 7, we present the real-time mapping relationship $\left(g^{X}\right)(\omega) \sim \omega$ of the considered mapped WENO schemes, where two of the non-OP points are selected and highlighted in solid symbols for demonstration. In Table 4, we present the computed values of the nonlinear weights, both before and after the mapping process, associated with these highlighted non-OP points. We also give the order (in brackets in descending manner) of these nonlinear weights. It is evident that the order of the nonlinear weights has been changed when the mapping process is implemented at each non-OP point. In addition, as shown in Fig. 8 and Fig. 9, we see that there are many non-OP points in the numerical solutions of the WENO-PM6 scheme for both short and long output times. Similarly, we also find many non-OP points in the results of the WENO-IM $(2,0.1)$ and MIP-WENO-ACM $k$ schemes, while we do not present them here just for brevity.

\subsubsection{Effects of the non-OP mapping process on the numerical solutions}

Without loss of generality, we assume that the weights $\omega_{s}^{\mathrm{JS}}$, which would be substituted into some mapping function, satisfy $\omega_{0}^{\mathrm{JS}}>\omega_{1}^{\mathrm{JS}}>\omega_{2}^{\mathrm{JS}}$, the mapped weights $\omega_{s}^{\text {non-OP }}$ computed by some set of mapping functions which 

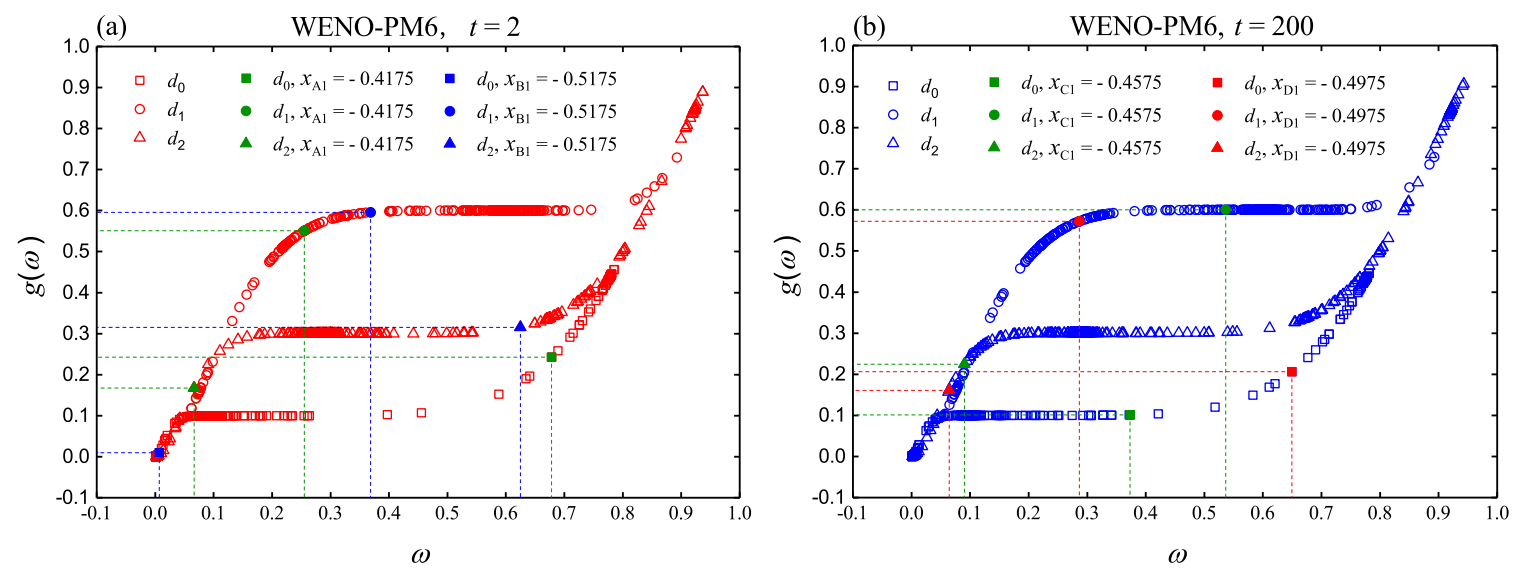

Fig. 5. The real-time mapping relationship $\left(g^{\mathrm{PM} 6}\right)(\omega) \sim \omega$ of the SLP. A uniform mesh size of $N=400$ and two output times $t=2$ (left) and $t=200$ (right) are used.
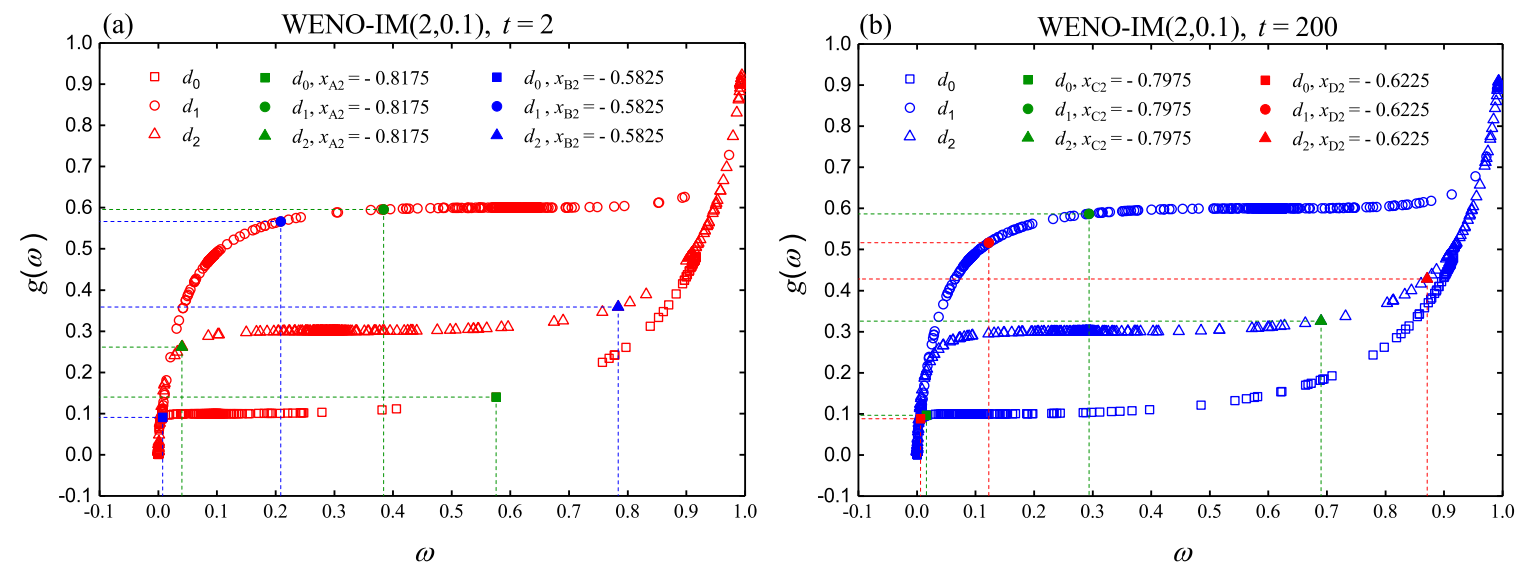

Fig. 6. The real-time mapping relationship $\left(g^{\operatorname{IM}(2,0.1)}\right)(\omega) \sim \omega$ of the SLP. A uniform mesh size of $N=400$ and two output times $t=2$ (left) and $t=200$ (right) are used.

is non-OP satisfy $\omega_{1}^{\text {non-OP }}>\omega_{2}^{\text {non-OP }}>\omega_{0}^{\text {non-OP }}$, and the mapped weights $\omega_{s}^{\mathrm{OP}}$ computed by some set of mapping functions which is $O P$ satisfy $\omega_{0}^{\mathrm{OP}}>\omega_{1}^{\mathrm{OP}}>\omega_{2}^{\mathrm{OP}}$.

According to Eq. 5 , by adding and subtracting $\sum_{s=0}^{2} d_{s}^{\text {non-OP }} u_{j+1 / 2}^{s}$ to $u_{j+1 / 2}^{\text {non-OP }}=\sum_{s=0}^{2} \omega_{s}^{\text {non-OP }} u_{j+1 / 2}^{s}$, we obtain

$$
u_{j+1 / 2}^{\mathrm{non}-\mathrm{OP}}=\sum_{s=0}^{2} d_{s}^{\mathrm{non}-\mathrm{OP}} u_{j+1 / 2}^{s}+\sum_{s=0}^{2}\left(\omega_{s}^{\mathrm{non}-\mathrm{OP}}-d_{s}^{\mathrm{non}-\mathrm{OP}}\right) u_{j+1 / 2}^{s} .
$$

In smooth regions, the first term in the right-hand side of Eq.(20) satisfies [15, 2, 8]

$$
\sum_{s=0}^{2} d_{s}^{\mathrm{non}-\mathrm{OP}} u_{j+1 / 2}^{s}=u\left(x_{j+1 / 2}\right)+O\left(\Delta x^{5}\right)
$$

Similarly, we have

$$
u_{j+1 / 2}^{\mathrm{OP}}=\sum_{s=0}^{2} d_{s}^{\mathrm{OP}} u_{j+1 / 2}^{s}+\sum_{s=0}^{2}\left(\omega_{s}^{\mathrm{OP}}-d_{s}^{\mathrm{OP}}\right) u_{j+1 / 2}^{s},
$$



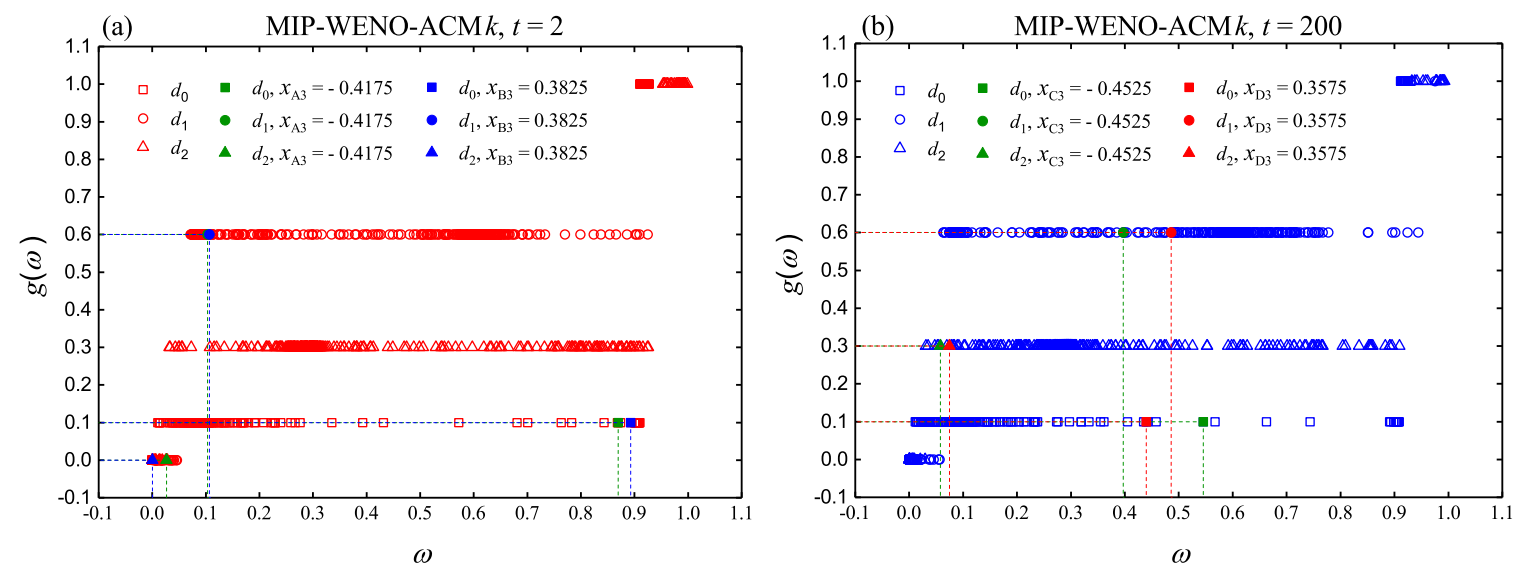

Fig. 7. The real-time mapping relationship $\left(g^{\mathrm{ACM} k}\right)(\omega) \sim \omega$ of the SLP. A uniform mesh size of $N=400$ and two output times $t=2$ (left) and $t=200$ (right) are used.

Table 4. The mapping results of the SLP on highlighted non-OP points, computed by WENO-PM6, WENO-IM $(2,0.1)$ and MIP-WENO-ACM $k$, with a uniform mesh size of $N=400$ and output times $t=2,200$.

\begin{tabular}{|c|c|c|c|c|c|c|c|c|c|}
\hline \multirow[b]{2}{*}{ Schemes, X } & \multirow[b]{2}{*}{ Time, $t$} & \multirow[b]{2}{*}{ Point } & \multirow[b]{2}{*}{ Position, $x$} & \multicolumn{3}{|c|}{ Before the mapping (the order) } & \multicolumn{3}{|c|}{ After the mapping (the order) } \\
\hline & & & & $\omega_{0}$ & $\omega_{1}$ & $\omega_{2}$ & $g_{0}\left(\omega_{0}\right)$ & $g_{1}\left(\omega_{1}\right)$ & $g_{2}\left(\omega_{2}\right)$ \\
\hline \multirow[t]{4}{*}{ WENO-PM6 } & 2 & A1 & -0.4175 & $0.67828(1)$ & $0.25528(2)$ & $0.06644(3)$ & $0.24252(2)$ & $0.55068(1)$ & $0.16737(3)$ \\
\hline & 2 & B1 & -0.5175 & $0.00678(3)$ & $0.36849(2)$ & $0.62473(1)$ & $0.00980(3)$ & $0.59595(1)$ & $0.31538(2)$ \\
\hline & 200 & $\mathrm{C} 1$ & -0.4575 & $0.37291(2)$ & $0.53663(1)$ & $0.09046(3)$ & $0.10125(3)$ & $0.60000(1)$ & $0.22432(2)$ \\
\hline & 200 & D1 & -0.4975 & $0.64949(1)$ & $0.28636(2)$ & $0.06415(3)$ & $0.20605(2)$ & $0.57222(1)$ & $0.16098(3)$ \\
\hline \multirow[t]{4}{*}{$\overline{\text { WENO-IM }(2,0.1)}$} & 2 & $\mathrm{~A} 2$ & -0.8175 & $0.57568(1)$ & $0.38416(2)$ & $0.04016(3)$ & $0.14033(3)$ & $0.59583(1)$ & $\overline{0.26127(2)}$ \\
\hline & 2 & B2 & -0.5825 & $0.00768(3)$ & $0.20854(2)$ & $0.78378(1)$ & $0.09071(3)$ & $0.56674(1)$ & $0.35871(2)$ \\
\hline & 200 & $\mathrm{C} 2$ & -0.7975 & $0.01609(3)$ & $0.29405(2)$ & $0.68986(1)$ & $0.09643(3)$ & $0.58680(1)$ & $0.32586(2)$ \\
\hline & 200 & $\mathrm{D} 2$ & -0.6225 & $0.00622(3)$ & $0.12285(2)$ & $0.87093(1)$ & $0.08832(3)$ & $0.51677(1)$ & $0.42834(2)$ \\
\hline \multirow[t]{4}{*}{ MIP-WENO-ACM $k$} & 2 & A3 & -0.4175 & $0.86977(1)$ & $0.10334(2)$ & $0.02689(3)$ & $0.10000(2)$ & $0.60000(1)$ & $0.00000(3)$ \\
\hline & 2 & B3 & 0.3825 & $0.89299(1)$ & $0.10671(2)$ & $0.00030(3)$ & $0.10000(2)$ & $0.60000(1)$ & $0.00000(3)$ \\
\hline & 200 & $\mathrm{C} 3$ & -0.4525 & $0.54547(1)$ & $0.39684(2)$ & $0.05769(3)$ & $0.10000(3)$ & $0.60000(1)$ & $0.30000(2)$ \\
\hline & 200 & D3 & 0.3575 & $0.43952(2)$ & $0.48568(1)$ & $0.07480(3)$ & $0.10000(3)$ & $0.60000(1)$ & $0.30000(2)$ \\
\hline
\end{tabular}

and

$$
\sum_{s=0}^{2} d_{s}^{\mathrm{OP}} u_{j+1 / 2}^{s}=u\left(x_{j+1 / 2}\right)+O\left(\Delta x^{5}\right) .
$$

So the second term in the right-hand side of Eq. 20 or Eq. 22 must be at least an $O\left(\Delta x^{6}\right)$ quantity to ensure the convergence rate to be approximated at 5th-order, and this is the key point that the mapped WENO methods have focused on.

However, in the parts of solutions with discontinuities, Eq.(21) and Eq.(23) usually do not hold. Furthermore, it is easy to know that the non-OP mapping process will amplify the effect from the relatively non-smooth stencils and decrease the effect from the relatively smooth stencils, so that we can probably get the following inequality

$$
\left|\sum_{s=0}^{2} d_{s}^{\mathrm{non}-\mathrm{OP}} u_{j+1 / 2}^{s}-u\left(x_{j+1 / 2}\right)\right|>\left|\sum_{s=0}^{2} d_{s}^{\mathrm{OP}} u_{j+1 / 2}^{s}-u\left(x_{j+1 / 2}\right)\right| .
$$

Now, we analyze the effect of the second term in the right-hand side of Eq.20) and Eq. (22). Suppose that $\left(g^{\text {non-OP }}\right)_{s}\left(d_{s}^{\text {non-OP }}\right)=d_{s}^{\text {nnn-OP }},\left(g^{\text {non-OP }}\right)_{s}^{\prime}\left(d_{s}^{\text {non-OP }}\right)=\cdots=\left(g^{\text {non-OP }}\right)_{s}^{(n-1)}\left(d_{s}^{\text {non-OP }}\right)=0,\left(g^{\text {non-OP }}\right)_{s}^{(n)}\left(d_{s}^{\text {non-OP }}\right) \neq 0$. 

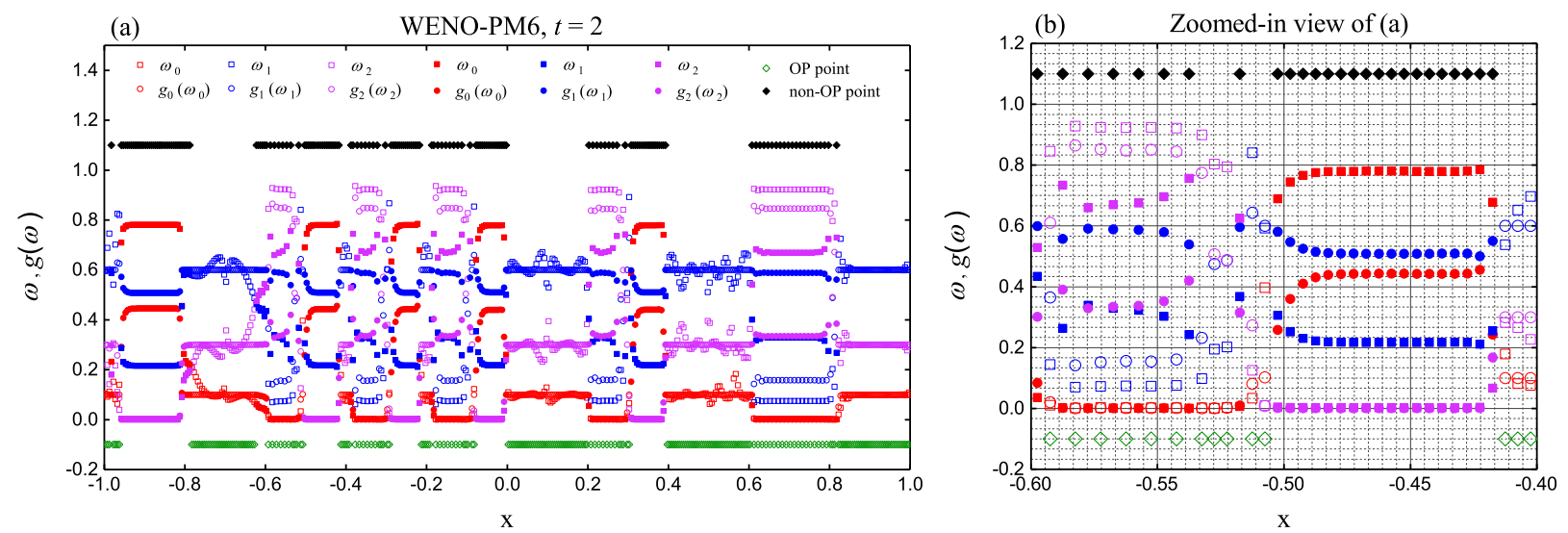

Fig. 8. The non-OP points in the numerical solutions of the WENO-PM6 scheme. A uniform mesh size of $N=400$ is used and the output time is $t=2$.
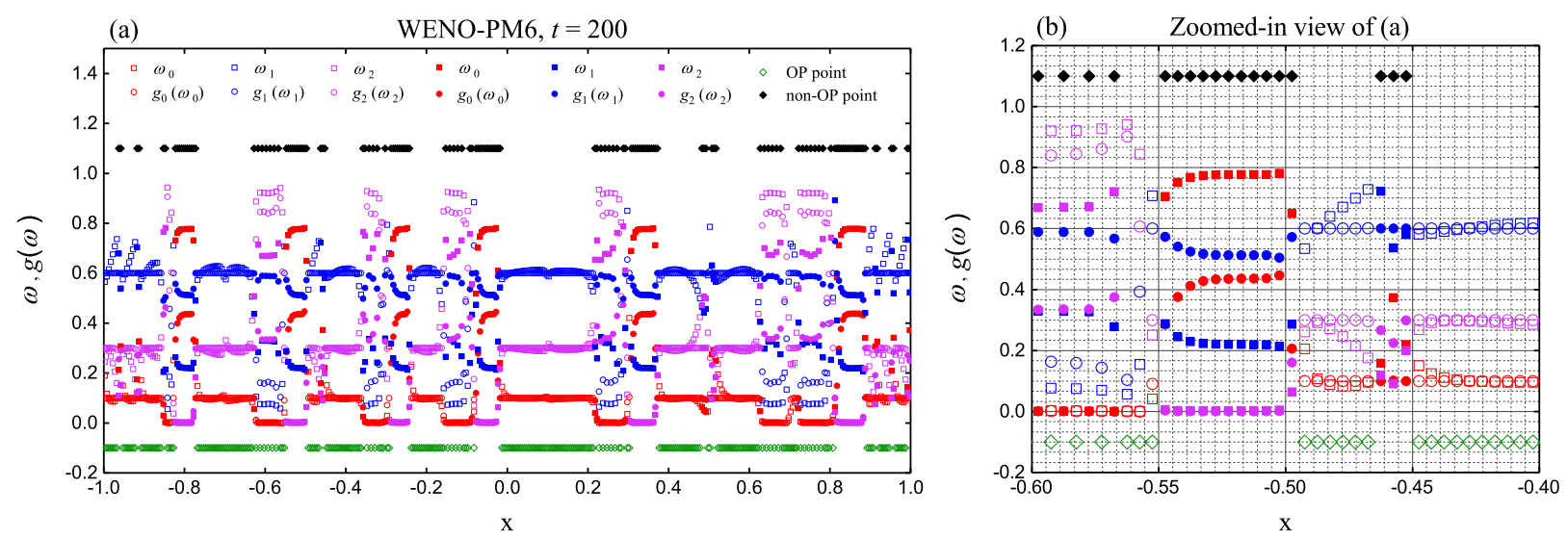

Fig. 9. The non-OP points in the numerical solutions of the WENO-PM6 scheme. A uniform mesh size of $N=400$ is used and the output time is $t=200$.

Then, evaluation at $\omega_{s}^{\mathrm{JS}}$ of the Taylor series approximations of $\left(g^{\text {non-OP }}\right)_{s}(\omega)$ about $d_{s}^{\text {non-OP }}$ yields

$$
\begin{aligned}
\alpha_{s}^{\mathrm{non}-\mathrm{OP}} & =\left(g^{\mathrm{non}-\mathrm{OP}}\right)_{s}\left(d_{s}^{\mathrm{non}-\mathrm{OP}}\right)+\left(g^{\mathrm{non}-\mathrm{OP}}\right)_{s}^{\prime}\left(d_{s}^{\mathrm{non}-\mathrm{OP}}\right)\left(\omega_{s}^{\mathrm{JS}}-d_{s}^{\mathrm{non}-\mathrm{OP}}\right)+\cdots \\
& +\frac{\left(g^{\mathrm{non}-\mathrm{OP}}\right)_{s}^{(n)}\left(d_{s}^{\mathrm{non}-\mathrm{OP}}\right)}{n !}\left(\omega_{s}^{\mathrm{JS}}-d_{s}^{\mathrm{non}-\mathrm{OP}}\right)^{n}+O\left(\left(\omega_{s}^{\mathrm{JS}}-d_{s}^{\mathrm{non}-\mathrm{OP}}\right)^{n+1}\right) \\
& =d_{s}^{\mathrm{non}-\mathrm{OP}}+\frac{\left(g^{\mathrm{non}-\mathrm{OP}}\right)_{s}^{(n)}\left(d_{s}^{\mathrm{non}-\mathrm{OP}}\right)}{n !}\left(\omega_{s}^{\mathrm{JS}}-d_{s}^{\mathrm{non}-\mathrm{OP}}\right)^{n}+O\left(\left(\omega_{s}^{\mathrm{JS}}-d_{s}^{\mathrm{non}-\mathrm{OP}}\right)^{n+1}\right)
\end{aligned}
$$

Similarly, when $\left(g^{\mathrm{OP}}\right)_{s}\left(d_{s}^{\mathrm{OP}}\right)=d_{s}^{\mathrm{OP}},\left(g^{\mathrm{OP}}\right)_{s}^{\prime}\left(d_{s}^{\mathrm{OP}}\right)=\cdots=\left(g^{\mathrm{OP}}\right)_{s}^{(n-1)}\left(d_{s}^{\mathrm{OP}}\right)=0,\left(g^{\mathrm{OP}}\right)_{s}^{(n)}\left(d_{s}^{\mathrm{OP}}\right) \neq 0$, we have

$$
\begin{aligned}
\alpha_{s}^{\mathrm{OP}} & =\left(g^{\mathrm{OP}}\right)_{s}\left(d_{s}^{\mathrm{OP}}\right)+\left(g^{\mathrm{OP}}\right)_{s}^{\prime}\left(d_{s}^{\mathrm{OP}}\right)\left(\omega_{s}^{\mathrm{JS}}-d_{s}^{\mathrm{OP}}\right)+\cdots+\frac{\left(g^{\mathrm{OP}}\right)_{s}^{(n)}\left(d_{s}^{\mathrm{OP}}\right)}{n !}\left(\omega_{s}^{\mathrm{JS}}-d_{s}^{\mathrm{OP}}\right)^{n}+O\left(\left(\omega_{s}^{\mathrm{JS}}-d_{s}^{\mathrm{OP}}\right)^{n+1}\right) \\
& =d_{s}^{\mathrm{OP}}+\frac{\left(g^{\mathrm{OP}}\right)_{s}^{(n)}\left(d_{s}^{\mathrm{OP}}\right)}{n !}\left(\omega_{s}^{\mathrm{JS}}-d_{s}^{\mathrm{OP}}\right)^{n}+O\left(\left(\omega_{s}^{\mathrm{JS}}-d_{s}^{\mathrm{OP}}\right)^{n+1}\right) .
\end{aligned}
$$


Then, from Eq.25) and Eq.(26), we obtain

$$
\frac{\alpha_{s}^{\mathrm{non}-\mathrm{OP}}-d_{s}^{\mathrm{non}-\mathrm{OP}}}{\alpha_{s}^{\mathrm{OP}}-d_{s}^{\mathrm{OP}}} \approx \frac{\left(g^{\mathrm{non}-\mathrm{OP}}\right)_{s}^{(n)}\left(d_{s}^{\mathrm{non}-\mathrm{OP}}\right)}{\left(g^{\mathrm{OP}}\right)_{s}^{(n)}\left(d_{s}^{\mathrm{OP}}\right)} \times\left(\frac{\omega_{s}^{\mathrm{JS}}-d_{s}^{\mathrm{non}-\mathrm{OP}}}{\omega_{s}^{\mathrm{JS}}-d_{s}^{\mathrm{OP}}}\right)^{n}
$$

One may probably get $\frac{\omega_{s}^{\mathrm{JS}}-d_{s}^{\text {non-OP }}}{\omega_{s}^{\mathrm{JS}}-d_{s}^{\mathrm{OP}}} \gg 1$ at a non-OP point where a non-OP mapping process occurs. Then, as $n$ is a possibly large positive integer, Eq.27) yields

$$
\alpha_{s}^{\text {non-OP }}-d_{s}^{\text {non-OP }} \gg \alpha_{s}^{\mathrm{OP}}-d_{s}^{\mathrm{OP}} .
$$

Thus, according to Eq.(46) in [15], it is trivial to know that

$$
\omega_{s}^{\mathrm{non}-\mathrm{OP}}-d_{s}^{\mathrm{non}-\mathrm{OP}} \gg \omega_{s}^{\mathrm{OP}}-d_{s}^{\mathrm{OP}} .
$$

Now, from Eq.20) 22, 24) 29, and considering the accumulation of the errors when $t$ gets larger, we can conclude that the non-OP mapping process might probably be the essential cause of the spurious oscillation generation and potential loss of accuracy when the mapped WENO schemes are used to simulate the problems with discontinuities for long output times.

To illustrate this, and for brevity in the discussion but without loss of generality, we assume that there is a global stencil $S^{5}$ which is divided into 3 substencils $S_{0}, S_{1}, S_{2}$, and there is an isolated discontinuity on $S_{2}$, as shown in Fig. 10. We suppose $u_{\mathrm{L}}=1, u_{\mathrm{R}}=-1$ and $u_{j+1 / 2}^{0}=u_{j+1 / 2}^{1}=1, u_{j+1 / 2}^{2}=-1$. According to Eq. (5), we calculate the approximation of $u\left(x_{j+1 / 2}\right)$ on the stencil $S^{5}$, by applying the mapped weights of the non-OP points C1, A2, C3 in Table 4 to the corresponding substencils in Fig. 10, respectively. For comparison, we also calculate the results by applying the weights of the WENO-JS scheme and the mapped weights of some OP points. Here, for simplicity but without loss of generality, we directly use the same values of the mapped weights of the non-OP points $\mathrm{C} 1$, A2, C3 in Table 4, but change their order, to set the values of the mapped weights of the OP points. We present the computing conditions and results in detail in Table 5. From Table 5, we find that the errors computed by using the mapped weights of the non-OP points are much larger than the solutions computed by using the mapped weights of the $O P$ points. Although we only present a pseudo-test example here, it is highly conducive to describe and understand the way that the non-OP mapping process causes the potential loss of accuracy. In practice tests with short output times, this phenomenon, as well as the spurious oscillation, may not be easy to be noticed. However, the errors are accumulated and will be demonstrated when the output time gets larger, and then the spurious oscillation and potential loss of accuracy can be observed. We will show these through numerical experiments in Section 5

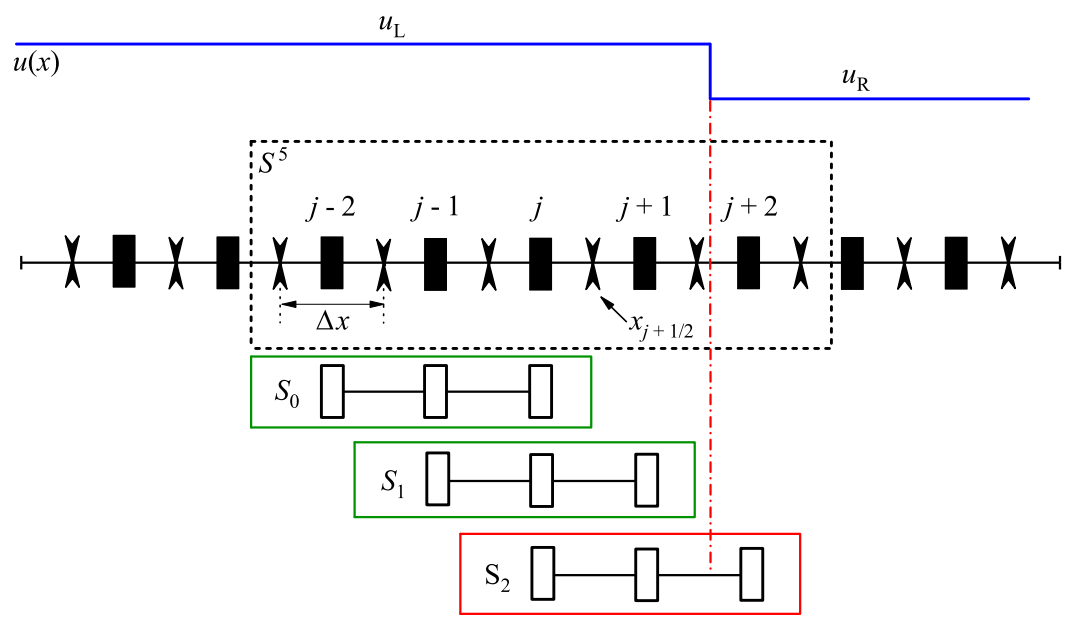

Fig. 10. Schematic of fifth-order WENO stencils with an isolated discontinuity on one of the substencils. 
Table 5. The computing conditions and results for comparison.

\begin{tabular}{llll}
\hline & WENO-PM6, Point C1 & WENO-IM $(2,0.1)$, Point A2 & MIP-WENO-ACM $k$ Point C3 \\
\hline$\left(\omega_{0}^{\mathrm{SS}}, \omega_{1}^{\mathrm{SS}}, \omega_{2}^{\mathrm{SS}}\right)$ & $(0.37291,0.53663,0.09046)$ & $(0.57568,0.38416,0.04016)$ & $(0.54547,0.39684,0.05769)$ \\
$\left(\omega_{0}^{\text {non-OP }}, \omega_{1}^{\text {non-OP }}, \omega_{2}^{\text {non-OP }}\right)$ & $(0.10939,0.64825,0.24236)$ & $(0.14069,0.59737,0.26194)$ & $(0.10000,0.60000,0.30000)$ \\
$\left(\omega_{0}^{\mathrm{OP}}, \omega_{1}^{\mathrm{OP}}, \omega_{2}^{\mathrm{OP}}\right)$ & $(0.24236,0.64825,0.10939)$ & $(0.59737,0.26194,0.14069)$ & $(0.60000,0.30000,0.10000)$ \\
$\left(u_{j+1 / 2}^{\mathrm{JS}}, u_{j+1 / 2}^{\text {non }-\mathrm{OP}}, u_{j+1 / 2}^{\mathrm{OP}}\right)$ & $(0.81908,0.51528,0.78122)$ & $(0.91968,0.47611,0.71862)$ & $(0.88462,0.40000,0.80000)$ \\
$u_{j+1 / 2}^{\mathrm{SS}}-u\left(x_{j+1 / 2}\right)$ & $0.18092(18.09 \%)$ & $0.08031(8.03 \%)$ & $0.11538(11.54 \%)$ \\
$u_{j+1 / 2}^{\text {non }-\mathrm{OP}}-u\left(x_{j+1 / 2}\right)$ & $0.48472(48.47 \%)$ & $0.52389(52.39 \%)$ & $0.60000(60.00 \%)$ \\
$u_{j+1 / 2}^{\mathrm{OP}}-u\left(x_{j+1 / 2}\right)$ & $0.21878(21.88 \%)$ & $0.28138(28.14 \%)$ & $0.20000(20.00 \%)$ \\
\hline
\end{tabular}

\section{Design and properties of the order-preserving mapping functions}

\subsection{Design of the new mapping function}

To design a new mapped WENO scheme that can prevent the non-OP mapping process, we devise a set of mapping functions that is OP.

Let $\mathcal{D}=\left\{d_{0}, d_{1}, \cdots, d_{r-1}\right\}$ be an array of all the ideal weights of the (2r-1)th-order WENO schemes. We build a new array by sorting the elements of $\mathcal{D}$ in ascending order, that is, $\widetilde{\mathcal{D}}=\left\{\widetilde{d}_{0}, \widetilde{d}_{1}, \cdots, \widetilde{d}_{r-1}\right\}$. In other words, the arrays $\mathcal{D}$ and $\widetilde{\mathcal{D}}$ have the same elements with different arrangements, and the elements of $\tilde{\mathcal{D}}$ satisfy

$$
\widetilde{d}_{0}<\widetilde{d}_{1}<\cdots<\widetilde{d}_{r-1} .
$$

The following notations are introduced to simplify the expressions

$$
\hat{\Omega}_{1}=\left[0, \mathrm{CFS}_{0}\right), \hat{\Omega}_{2}=\left[\mathrm{CFS}_{0}, \frac{\widetilde{d}_{0}+\widetilde{d}_{1}}{2}\right), \cdots, \hat{\Omega}_{r+1}=\left[\frac{\widetilde{d}_{r-2}+\widetilde{d}_{r-1}}{2}, \mathrm{CFS}_{1}\right], \hat{\Omega}_{r+2}=\left(\mathrm{CFS}_{1}, 1\right],
$$

where $0<\mathrm{CFS}_{0} \leq \widetilde{d}_{0}$ and $\widetilde{d}_{r-1} \leq \mathrm{CFS}_{1} \leq 1$. It is easy to verify that: (1) $\hat{\Omega}=\hat{\Omega}_{1} \cup \hat{\Omega}_{2} \cup \cdots \cup \hat{\Omega}_{r+2}$; (2) if $i \neq j$, then $\hat{\Omega}_{i} \cap \hat{\Omega}_{j}=\varnothing, \forall i, j=1,2, \cdots, r+2$.

Now, we give a new mapping function as follows

$$
\left(g^{\mathrm{MOP}-\mathrm{ACM} k}\right)_{s}(\omega)= \begin{cases}k_{0} \omega, & \omega \in \hat{\Omega}_{1}, \\ \widetilde{d}_{0}, & \omega \in \hat{\Omega}_{2}, \\ \widetilde{d}_{1}, & \omega \in \hat{\Omega}_{3}, \\ & \vdots \\ \widetilde{d}_{r-1}, & \omega \in \hat{\Omega}_{r+1}, \\ 1-k_{1}(1-\omega), & \omega \in \hat{\Omega}_{r+2},\end{cases}
$$

where

$$
k_{0} \in\left[0, \frac{\widetilde{d}_{0}}{\mathrm{CFS}_{0}}\right], k_{1} \in\left[0, \frac{1-\widetilde{d}_{r-1}}{1-\mathrm{CFS}_{1}}\right] .
$$

Actually, we can verify that $\left(g^{\mathrm{MOP}-\mathrm{ACM} k}\right)_{s}(\omega)$ is independent of the parameter $s$, that is, $\left(g^{\mathrm{MOP}-\mathrm{ACM} k}\right)_{0}(\omega)=$ $\cdots=\left(g^{\mathrm{MOP}-\mathrm{ACM} k}\right)_{r-1}(\omega)$, which is significantly different from the previously published mapping functions. Thus, for simplicity, we will drop the subscript $s$ of $\left(g^{\mathrm{MOP}-\mathrm{ACM} k}\right)_{s}(\omega)$ in the following.

\subsection{Properties of the new mapping function}

Lemma 6. The mapping function $\mathrm{g}^{\mathrm{MOP}-\mathrm{ACM} k}(\omega)$ defined by Eq. 32, is order-preserving $(O P)$.

Proof. According to Eq. $30,31,32$, we can easily verify that $g^{\operatorname{MOP}-\operatorname{ACM} k}(\omega)$ satisfies Definition 2 .

As mentioned earlier, $g^{\mathrm{MOP}-\mathrm{ACM} k}(\omega)$ is a monotone increasing piecewise mapping function. It satisfies the following properties. 
Lemma 7. Let $\bar{\Omega}_{i}=\left\{\omega \in \hat{\Omega}_{i}\right.$ and $\left.\omega \neq \partial \hat{\Omega}_{i}\right\}$, then the mapping function $g^{\mathrm{MOP}-\mathrm{ACM} k}(\omega)$ defined by Eq. 32 , satisfies the following properties:

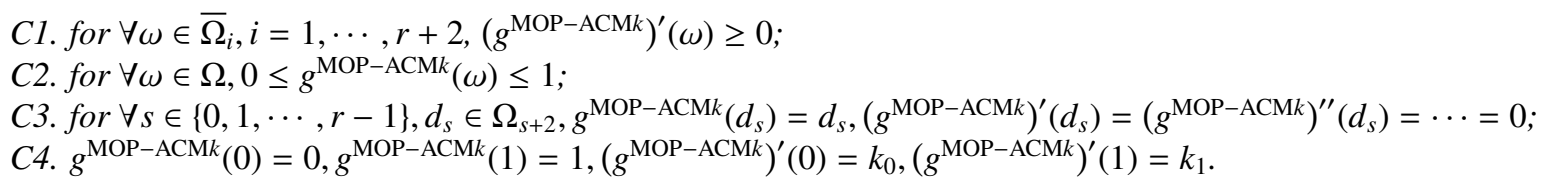

As the proof of Lemma 7 is very easy, we do not state them here and we can observe these properties intuitively in Fig. 11

Now, we give the new mapped WENO scheme with monotone increasing piecewise and order-preserving mapping, denoted as MOP-WENO-ACM $k$. The mapped weights are given by

$$
\omega_{s}^{\mathrm{MOP}-\mathrm{ACM} k}=\frac{\alpha_{s}^{\mathrm{MOP}-\mathrm{ACM} k}}{\sum_{l=0}^{r-1} \alpha_{l}^{\mathrm{MOP}-\mathrm{ACM} k}}, \alpha_{s}^{\mathrm{MOP}-\mathrm{ACM} k}=g^{\mathrm{MOP}-\mathrm{ACM} k}\left(\omega_{s}^{\mathrm{JS}}\right) .
$$

We present Theorem 2, which will show that the MOP-WENO-ACM $k$ scheme can recover the optimal convergence rates for different values of $n_{\mathrm{cp}}$ in smooth regions.

Theorem 2. When $\mathrm{CFS}_{0} \ll \widetilde{d}_{0}$ and $\mathrm{CFS}_{1} \gg \widetilde{d}_{r-1}$, for $\forall n_{\mathrm{cp}}<r-1$, the $(2 r-1)$ th-order MOP-WENO-ACMk scheme can achieve the optimal convergence rate of accuracy if the mapping function $g^{\mathrm{MOP}-\mathrm{ACM} k}(\omega)$ is applied to the weights of the $(2 r-1)$ th-order WENO-JS scheme.

We can prove Theorem 2 by employing the Taylor series analysis and using Lemma 7 of this paper and Lemma 1 and Lemma 2 in the statement of page 456 to 457 in [8]. The detailed proof process is almost identical to the one in [15].

We can get the fifth-order MOP-WENO-ACM $k$ scheme by choosing $r=3$ in Eq.(32) (34). In this case, it is trivial to obtain the arrays $\mathcal{D}, \widetilde{\mathcal{D}}$, that is, $\mathcal{D}=\{0.1,0.6,0.3\}, \widetilde{\mathcal{D}}=\{0.1,0.3,0.6\}$. In other words, we have $\widetilde{d}_{0}=0.1, \widetilde{d}_{1}=$ $0.3, \widetilde{d}_{2}=0.6$. Then, we can write the mapping function explicitly as follows

$$
g^{\mathrm{MOP}-\mathrm{ACM} k}(\omega)= \begin{cases}k_{0} \omega, & \omega \in \hat{\Omega}_{1}, \\ 0.1, & \omega \in \hat{\Omega}_{2}, \\ 0.3, & \omega \in \hat{\Omega}_{3}, \\ 0.6, & \omega \in \hat{\Omega}_{4}, \\ 1-k_{1}(1-\omega), & \omega \in \hat{\Omega}_{5},\end{cases}
$$

where $\hat{\Omega}_{1}=\left[0, \mathrm{CFS}_{0}\right), \hat{\Omega}_{2}=\left[\mathrm{CFS}_{0}, 0.2\right), \hat{\Omega}_{3}=[0.2,0.45), \hat{\Omega}_{4}=\left[0.45, \mathrm{CFS}_{1}\right], \hat{\Omega}_{5}=\left(\mathrm{CFS}_{1}, 1\right], 0<\mathrm{CFS}_{0} \leq 0.1,0.6 \leq$ $\mathrm{CFS}_{1}<1, k_{0} \in\left[0, \frac{0.1}{\mathrm{CFS}_{0}}\right], k_{1} \in\left[0, \frac{0.4}{1-\mathrm{CFS}_{1}}\right]$.

In Fig. 11, we plot the curve of $g^{\mathrm{MOP}-\mathrm{ACM} k}(\omega)$ varying with $\omega$ by taking $\mathrm{CFS}_{0}=0.04, \mathrm{CFS}_{1}=0.92$ and $k_{0}=$ $2.5, k_{1}=5$. 


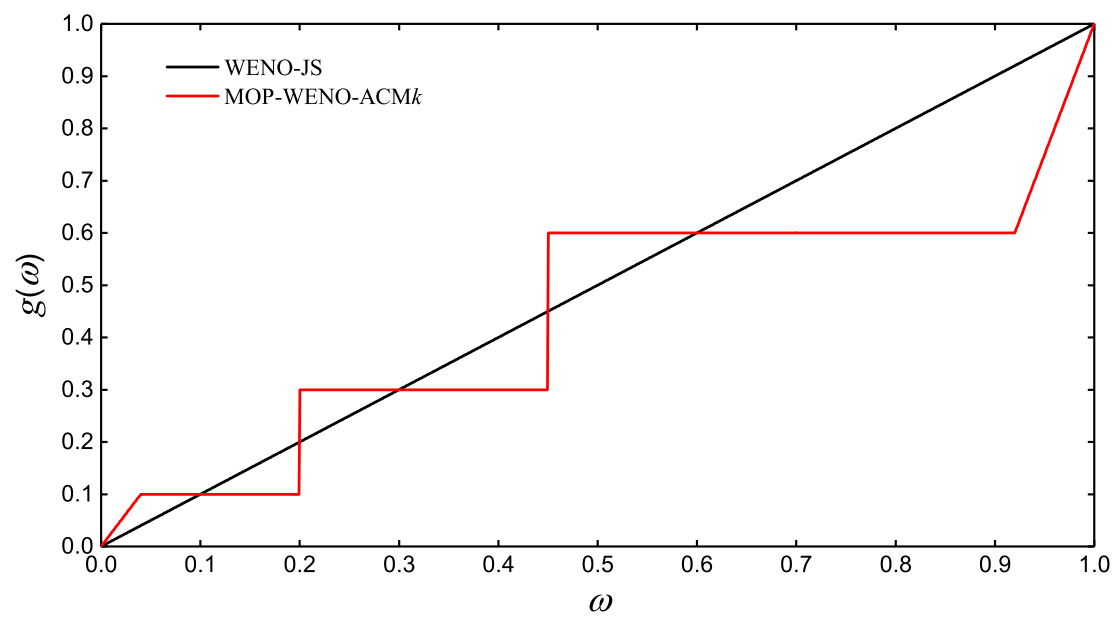

Fig. 11. The mapping function of the MOP-WENO-ACM $k$ scheme with $\mathrm{CFS}_{0}=0.04, \mathrm{CFS}_{1}=0.92, k_{0}=2.5, k_{1}=5$.

\section{Numerical experiments}

In this section, we compare the numerical performance of the MOP-WENO-ACM $k$ scheme with the WENO-JS scheme [17] and its various versions with mapping, WENO-M [15], WENO-PM6 [7], WENO-IM(2, 0.1) [8] and MIP-WENO-ACM $k$ which is proposed in subsection 3.1 In all the numerical experiments below, MOP-WENO$\mathrm{ACM} k$ refers to the definition in Eq. (34) and Eq. (35) with $k_{0}=k_{1}=0, \mathrm{CFS}_{0}=0.01, \mathrm{CFS}_{1}=0.94$, and the parameters in the MIP-WENO-ACM $k$ scheme are chosen to be $k_{s}=0, \mathrm{CFS}_{s}=d_{s} / 10$.

The numerical presentation of this section starts with the accuracy test of the one-dimensional linear advection equation with four kinds of initial conditions, followed by the solutions at long output times of one-dimensional linear advection equation with two kinds of initial condition with discontinuities, and finishes with 2D calculations on the two-dimensional Riemann problem and the shock-vortex interaction.

\subsection{Accuracy test}

Example 1. (Accuracy test without critical points [8]) We consider the one-dimensional linear advection equation Eq.(14) with the periodic boundary condition and the following initial condition

$$
u(x, 0)=\sin (\pi x) .
$$

It is easy to know that the initial condition in Eq. 36 ) has no critical points. The CFL number is set to be $(\Delta x)^{2 / 3}$ to prevent the convergence rates of error from being influenced by time advancement. The $L_{1}, L_{2}, L_{\infty}$ norms of the error are given as

$$
\begin{aligned}
L_{1} & =h \cdot \sum_{j}\left|u_{j}^{\text {exact }}-\left(u_{h}\right)_{j}\right|, \\
L_{2} & =\sqrt{h \cdot \sum_{j}\left(u_{j}^{\text {exact }}-\left(u_{h}\right)_{j}\right)^{2},} \\
L_{\infty} & =\max _{j}\left|u_{j}^{\text {exact }}-\left(u_{h}\right)_{j}\right|,
\end{aligned}
$$

where $h=\Delta x$ is the uniform spatial step size, $\left(u_{h}\right)_{j}$ is the numerical solution and $u_{j}^{\text {exact }}$ is the exact solution.

The $L_{1}, L_{2}, L_{\infty}$ errors and corresponding convergence orders of various considered WENO schemes for Example 1 at output time $t=2.0$ are shown in Table 6 . The results of the three rows are $L_{1^{-}}, L_{2^{-}}$and $L_{\infty}$ - norm errors and convergence orders in turn (similarly hereinafter). Unsurprisingly, the MOP-WENO-ACM $k$ scheme has gained the fifth-order convergence like the other considered schemes. It can be found that the MOP-WENO-ACM $k$, MIPWENO-ACM $k$, WENO-M, WENO-PM6 and WENO-IM $(2,0.1)$ schemes give more accurate numerical solutions than the WENO-JS scheme in general. 
Table 6. Convergence properties of considered schemes solving $u_{t}+u_{x}=0$ with initial condition $u(x, 0)=\sin (\pi x)$.

\begin{tabular}{|c|c|c|c|c|c|c|}
\hline$N$ & 10 & 20 & 40 & 80 & 160 & 320 \\
\hline \multirow{3}{*}{ WENO-JS } & $6.18628 \mathrm{e}-02(-)$ & $2.96529 \mathrm{e}-03(4.3821)$ & $9.27609 \mathrm{e}-05(4.9985)$ & $2.89265 \mathrm{e}-06(5.0031)$ & $9.03392 \mathrm{e}-08(5.0009)$ & $2.82330 \mathrm{e}-09(4.9999)$ \\
\hline & $4.72306 \mathrm{e}-02(-)$ & $2.42673 \mathrm{e}-03(4.2826)$ & $7.64332 \mathrm{e}-05(4.9887)$ & $2.33581 \mathrm{e}-06(5.0322)$ & $7.19259 \mathrm{e}-08(5.0213)$ & $2.23105 \mathrm{e}-09(5.0107)$ \\
\hline & $4.87580 \mathrm{e}-02(-)$ & $2.57899 \mathrm{e}-03(4.2408)$ & $9.05453 \mathrm{e}-05(4.8320)$ & $2.90709 \mathrm{e}-06(4.9610)$ & $8.85753 \mathrm{e}-08(5.0365)$ & $2.72458 \mathrm{e}-09(5.0228)$ \\
\hline \multirow[t]{3}{*}{ WENO-M } & $2.01781 \mathrm{e}-02(-)$ & $5.18291 \mathrm{e}-04(5.2829)$ & $1.59422 \mathrm{e}-05(5.0228)$ & 4.98914e-07(4.9979) & $1.56021 \mathrm{e}-08(4.9990)$ & $4.99356 \mathrm{e}-10(4.9977)$ \\
\hline & $1.55809 \mathrm{e}-02(-)$ & $4.06148 \mathrm{e}-04(5.2616)$ & $1.25236 \mathrm{e}-05(5.0193)$ & $3.91875 \mathrm{e}-07(4.9981)$ & $1.22541 \mathrm{e}-08(4.9991)$ & $3.83568 \mathrm{e}-10(4.9976)$ \\
\hline & $1.47767 \mathrm{e}-02(-)$ & $3.94913 \mathrm{e}-04(5.2256)$ & $1.24993 \mathrm{e}-05(4.9816)$ & $3.91808 \mathrm{e}-07(4.9956)$ & $1.22538 \mathrm{e}-08(4.9988)$ & $3.83541 \mathrm{e}-10(4.9977)$ \\
\hline \multirow[t]{3}{*}{ WENO-PM6 } & $1.74869 \mathrm{e}-02(-)$ & $5.02923 \mathrm{e}-04(5.1198)$ & $1.59130 \mathrm{e}-05(4.9821)$ & $4.98858 \mathrm{e}-07(4.9954)$ & $1.56020 \mathrm{e}-08(4.9988)$ & $4.88355 \mathrm{e}-10(4.9977)$ \\
\hline & $1.35606 \mathrm{e}-02(-)$ & $3.95215 \mathrm{e}-04(5.1006)$ & $1.25010 \mathrm{e}-05(4.9825)$ & $3.91831 \mathrm{e}-07(4.9957)$ & $1.22541 \mathrm{e}-08(4.9989)$ & $3.83568 \mathrm{e}-10(4.9976)$ \\
\hline & $1.27577 \mathrm{e}-02(-)$ & $3.94515 \mathrm{e}-04(5.0151)$ & $1.24960 \mathrm{e}-05(4.9805)$ & $3.91795 \mathrm{e}-07(4.9952)$ & $1.22538 \mathrm{e}-08(4.9988)$ & $3.83543 \mathrm{e}-10(4.9977)$ \\
\hline \multirow[t]{3}{*}{ WENO-IM $(2,0.1)$} & $1.58051 \mathrm{e}-02(-)$ & $5.04401 \mathrm{e}-04(4.9697)$ & $1.59160 \mathrm{e}-05(4.9860)$ & $4.98863 \mathrm{e}-07(4.9957)$ & $1.56020 \mathrm{e}-08(4.9988)$ & $4.88355 \mathrm{e}-10(4.9977)$ \\
\hline & $1.23553 \mathrm{e}-02(-)$ & $3.96236 \mathrm{e}-04(4.9626)$ & $1.25033 \mathrm{e}-05(4.9860)$ & $3.91836 \mathrm{e}-07(4.9959)$ & $1.22541 \mathrm{e}-08(4.9989)$ & $3.83568 \mathrm{e}-10(4.9976)$ \\
\hline & $1.19178 \mathrm{e}-02(-)$ & $3.94458 \mathrm{e}-04(4.9171)$ & $1.24963 \mathrm{e}-05(4.9803)$ & $3.91797 \mathrm{e}-07(4.9953)$ & $1.22538 \mathrm{e}-08(4.9988)$ & $3.83547 \mathrm{e}-10(4.9977)$ \\
\hline \multirow[t]{3}{*}{ MIP-WENO-ACM $k$} & $1.52184 \mathrm{e}-02(-)$ & $5.02844 \mathrm{e}-04(4.9196)$ & $1.59130 \mathrm{e}-05(4.9818)$ & $4.98858 \mathrm{e}-07(4.9954)$ & $1.56020 \mathrm{e}-08(4.9988)$ & $4.88355 \mathrm{e}-10(4.9977)$ \\
\hline & $1.19442 \mathrm{e}-02(-)$ & $3.95138 \mathrm{e}-04(4.9178)$ & $1.25010 \mathrm{e}-05(4.9822)$ & $3.91831 \mathrm{e}-07(4.9957)$ & $1.22541 \mathrm{e}-08(4.9989)$ & $3.83568 \mathrm{e}-10(4.9976)$ \\
\hline & $1.17569 \mathrm{e}-02(-)$ & $3.94406 \mathrm{e}-04(4.8977)$ & $1.24960 \mathrm{e}-05(4.9801)$ & $3.91795 \mathrm{e}-07(4.9952)$ & $1.22538 \mathrm{e}-08(4.9988)$ & $3.83543 \mathrm{e}-10(4.9977)$ \\
\hline \multirow[t]{3}{*}{ MOP-WENO-ACM $k$} & $3.29609 \mathrm{e}-02(-)$ & $5.02844 \mathrm{e}-04(6.0345)$ & $1.59130 \mathrm{e}-05(4.9818)$ & $4.98858 \mathrm{e}-07(4.9954)$ & $1.56020 \mathrm{e}-08(4.9988)$ & $4.88355 \mathrm{e}-10(4.9977)$ \\
\hline & $2.72363 \mathrm{e}-02(-)$ & $3.95138 \mathrm{e}-04(6.1070)$ & $1.25010 \mathrm{e}-05(4.9822)$ & $3.91831 \mathrm{e}-07(4.9957)$ & $1.22541 \mathrm{e}-08(4.9989)$ & $3.83568 \mathrm{e}-10(4.9976$ \\
\hline & $2.70295 \mathrm{e}-02(-)$ & $3.94406 \mathrm{e}-04(6.0987)$ & $1.24960 \mathrm{e}-05(4.9801)$ & $3.91795 \mathrm{e}-07(4.9952)$ & $1.22538 \mathrm{e}-08(4.9988)$ & $3.83543 \mathrm{e}-10(4.9977$ \\
\hline
\end{tabular}

Example 2. (Accuracy test with first-order critical points [15]) We consider the one-dimensional linear advection equation Eq. (14) with the periodic boundary condition and the following initial condition

$$
u(x, 0)=\sin \left(\pi x-\frac{\sin (\pi x)}{\pi}\right) .
$$

The particular initial condition Eq. (37) has two first-order critical points, which both have a non-vanishing third derivative. As mentioned earlier, the CFL number is set to be $(\Delta x)^{2 / 3}$.

Table 7 shows the $L_{1}, L_{2}, L_{\infty}$ errors and corresponding convergence orders of the various considered WENO schemes at output time $t=2.0$. We can see that the $L_{\infty}$ convergence order of the WENO-JS scheme drops by almost 2 orders leading to an overall accuracy loss shown with $L_{1}$ and $L_{2}$ convergence orders. It is evident that the MOP-WENO-ACM $k$, MIP-WENO-ACM $k$, WENO-M, WENO-PM6 and WENO-IM(2,0.1) schemes can retain the optimal orders even in the presence of critical points. It is noteworthy that when the grid number is too small, like $N \leq 40$, in terms of accuracy, the MOP-WENO-ACM $k$ scheme provides less accurate results than those of the MIP-WENO-ACM $k$ scheme. The cause of this kind of accuracy loss is that the mapping function of the MOPWENO-ACM $k$ scheme has narrower optimal weight intervals (standing for the intervals about $\omega=d_{s}$ over which the mapping process attempts to use the corresponding optimal weights, see [19, 20]) than the MIP-WENO-ACM $k$ scheme. However, this issue can surely be overcome by increasing the grid number. Therefore, we can find that, as expected, the MOP-WENO-ACM $k$ scheme gives equally accurate numerical solutions like those of the MIP-WENOACM $k$ scheme when the grid number $N \geq 80$.

Table 7. Convergence properties of considered schemes solving $u_{t}+u_{x}=0$ with initial condition $u(x, 0)=\sin (\pi x-$ $\sin (\pi x) / \pi)$.

\begin{tabular}{|c|c|c|c|c|c|c|}
\hline $\bar{N}$ & 10 & 20 & 40 & 80 & 160 & 320 \\
\hline \multirow[t]{3}{*}{ WENO-JS } & $1.24488 \mathrm{e}-01(-)$ & $1.01260 \mathrm{e}-02(3.6199)$ & $7.22169 \mathrm{e}-04(3.8096)$ & $3.42286 \mathrm{e}-05(4.3991)$ & $1.58510 \mathrm{e}-06(4.4326)$ & $7.95517 \mathrm{e}-08(4.3165)$ \\
\hline & $1.09463 \mathrm{e}-01(-)$ & $8.72198 \mathrm{e}-03(3.6496)$ & $6.76133 \mathrm{e}-04(3.6893)$ & $3.63761 \mathrm{e}-05(4.2162)$ & $2.29598 \mathrm{e}-06(3.9858)$ & $1.68304 \mathrm{e}-07(3.7700)$ \\
\hline & $1.24471 \mathrm{e}-01(-)$ & $1.43499 \mathrm{e}-02(3.1167)$ & $1.09663 \mathrm{e}-03(3.7099)$ & $9.02485 \mathrm{e}-05(3.6030)$ & $8.24022 \mathrm{e}-06(3.4531)$ & $8.31702 \mathrm{e}-07(3.3085)$ \\
\hline \multirow[t]{3}{*}{ WENO-M } & $7.53259 \mathrm{e}-02(-)$ & $3.70838 \mathrm{e}-03(4.3443)$ & $1.45082 \mathrm{e}-04(4.6758)$ & $4.80253 \mathrm{e}-06(4.9169)$ & $1.52120 \mathrm{e}-07(4.9805)$ & $4.77083 \mathrm{e}-09(4.9948)$ \\
\hline & $6.39017 \mathrm{e}-02(-)$ & $3.36224 \mathrm{e}-03(4.2484)$ & $1.39007 \mathrm{e}-04(4.5962)$ & $4.52646 \mathrm{e}-06(4.9406)$ & $1.42463 \mathrm{e}-07(4.9897)$ & $4.45822 \mathrm{e}-09(4.9980)$ \\
\hline & $7.49250 \mathrm{e}-02(-)$ & $5.43666 \mathrm{e}-03(3.7847)$ & $2.18799 \mathrm{e}-04(4.6350)$ & $6.81451 \mathrm{e}-06(5.0049)$ & $2.14545 \mathrm{e}-07(4.9893)$ & $6.71080 \mathrm{e}-09(4.9987)$ \\
\hline \multirow[t]{3}{*}{ WENO-PM6 } & $9.51313 \mathrm{e}-02(-)$ & $4.82173 \mathrm{e}-03(4.3023)$ & $1.55428 \mathrm{e}-04(4.9552)$ & $4.87327 \mathrm{e}-06(4.9952)$ & $1.52750 \mathrm{e}-07(4.9956)$ & $4.77729 \mathrm{e}-09(4.9988)$ \\
\hline & $7.83600 \mathrm{e}-02(-)$ & 4.29 & $1.43841 \mathrm{e}-04(4.900$ & $36 \mathrm{e}-06(4.9855)$ & $1.42488 \mathrm{e}-07(4.9$ & 4.4580 \\
\hline & 9.323 & 5.91 & 2.09 & 6.8 & 2.14 & 6.7107 \\
\hline \multirow[t]{3}{*}{ WENO-IM(2,0.1) } & $8.38131 \mathrm{e}-02(-)$ & $4.30725 \mathrm{e}-03(4.2823)$ & $1.51327 \mathrm{e}-04(4.8310)$ & $4.85592 \mathrm{e}-06(4.9618)$ & $1.52659 \mathrm{e}-07(4.9914)$ & $4.77654 \mathrm{e}-09(4.9982)$ \\
\hline & $6.71285 \mathrm{e}-02(-)$ & $3.93700 \mathrm{e}-03(4.0918)$ & $1.41737 \mathrm{e}-04(4.7958)$ & $4.53602 \mathrm{e}-06(4.9656)$ & $1.42479 \mathrm{e}-07(4.9926)$ & $4.45805 \mathrm{e}-09(4.9982)$ \\
\hline & $7.62798 \mathrm{e}-02(-)$ & $5.84039 \mathrm{e}-03(3.7072)$ & $2.10531 \mathrm{e}-04(4.7940)$ & $6.82606 \mathrm{e}-06(4.9468)$ & $2.14534 \mathrm{e}-07(4.9918)$ & $6.71079 \mathrm{e}-09(4.9986)$ \\
\hline \multirow[t]{3}{*}{ MIP-WENO-ACM $k$} & $8.75629 \mathrm{e}-02(-)$ & $4.39527 \mathrm{e}-03(4.3163)$ & $1.52219 \mathrm{e}-04(4.8517)$ & $4.86436 \mathrm{e}-06(4.9678)$ & $1.52735 \mathrm{e}-07(4.9931)$ & $4.77728 \mathrm{e}-09(4.9987)$ \\
\hline & $6.98131 \mathrm{e}-02(-)$ & $4.02909 \mathrm{e}-03(4.1150)$ & $1.42172 \mathrm{e}-04(4.8247)$ & $4.53770 \mathrm{e}-06(4.9695)$ & $1.42486 \mathrm{e}-07(4.9931)$ & $4.45807 \mathrm{e}-09(4.9983)$ \\
\hline & $7.91292 \mathrm{e}-02(-)$ & $5.89045 \mathrm{e}-03(3.7478)$ & $2.09893 \mathrm{e}-04(4.8107)$ & $6.83017 \mathrm{e}-06(4.9416)$ & $2.14533 \mathrm{e}-07(4.9926)$ & $6.71079 \mathrm{e}-09(4.9986)$ \\
\hline \multirow[t]{3}{*}{ MOP-WENO-ACM $k$} & $9.08634 \mathrm{e}-02(-)$ & 7.09246e-03(3.6793) & $2.59429 \mathrm{e}-04(4.7729)$ & $4.86436 \mathrm{e}-06(5.7369)$ & $1.52735 \mathrm{e}-07(4.9931)$ & $4.77728 \mathrm{e}-09(4.9987)$ \\
\hline & $7.58160 \mathrm{e}-02(-)$ & $6.88532 \mathrm{e}-03(3.4609)$ & $2.51208 \mathrm{e}-04(4.7766)$ & $4.53770 \mathrm{e}-06(5.7908)$ & $1.42486 \mathrm{e}-07(4.9931)$ & $4.45807 \mathrm{e}-09(4.9983)$ \\
\hline & $9.29135 \mathrm{e}-02(-)$ & $1.01479 \mathrm{e}-02(3.1947)$ & $4.03069 \mathrm{e}-04(4.6540)$ & $6.83017 \mathrm{e}-06(5.8830)$ & $2.14533 \mathrm{e}-07(4.9926)$ & $6.71079 \mathrm{e}-09(4.9986)$ \\
\hline
\end{tabular}


Example 3. (High resolution performance test with high-order critical points) We consider the one-dimensional linear advection equation Eq.(14] with the periodic boundary condition and the following initial condition [7]

$$
u(x, 0)=\sin ^{9}(\pi x)
$$

It is trivial to verify that the initial condition in Eq. (38) has high-order critical points. Again, the CFL number is set to be $(\Delta x)^{2 / 3}$.

We calculate this problem using the MOP-WENO-ACM $k$, MIP-WENO-ACM $k$, WENO-JS and WENO-M schemes. Table 8 shows the $L_{1}, L_{2}, L_{\infty}$ errors of these WENO schemes at several output times with a uniform mesh size of $\Delta x=1 / 200$. We also present the corresponding increased errors (in percentage) compared to the errors of the MIPWENO-ACM $k$ scheme which gives the most accurate results. Taking the $L_{1}$-norm error as an example, its increased error at output time $t$ is calculated by $\frac{L_{1}^{X}(t)-L_{1}^{*}(t)}{L_{1}^{*}(t)} \times 100 \%$, where $L_{1}^{*}(t)$ and $L_{1}^{\mathrm{X}}(t)$ are the $L_{1}$-norm error of the MIPWENO-ACM $k$ scheme and the WENO-X scheme (X = WENO-JS, WENO-M, or MOP-WENO-ACM $k$ ) at output time $t$. Clearly, the WENO-JS scheme gives the largest increased errors for both short and long output times. At short output times, like $t \leq 100$, the solutions computed by the WENO-M scheme are most close to those of the MIP-WENO-ACM $k$ scheme, leading to the smallest increased errors. However, when the output time increases to $t \geq 200$, the solutions computed by the MOP-WENO-ACM $k$ scheme are most close to those of the MIP-WENO$\mathrm{ACM} k$ scheme. Furthermore, when the output time is large, the errors of the WENO-M scheme increase significantly leading to evidently larger increased errors, while the increased errors of the MOP-WENO-ACM $k$ scheme do not get larger as it provides comparably small errors. The errors of the MOP-WENO-ACM $k$ are not as small as those of the MIP-WENO-ACM $k$ scheme. The cause of this kind of accuracy loss is the same as stated in Example 2 that the mapping function of the MOP-WENO-ACM $k$ scheme has narrower optimal weight intervals than the MIP-WENO-ACM $k$ scheme. As mentioned earlier, one can surely address this issue by increasing the grid number. In order to verify this, we calculate this problem using the same schemes at the same output times but with a larger grid number of $N=800$, and the results are shown in Table 9 From Table 9 we can see that the errors of the MOP-WENO-ACM $k$ scheme are closer to those of the MIP-WENO-ACM $k$ scheme when the grid number increases from $N=200$ to $N=800$, resulting in the significantly decreasing of the increased errors. Moreover, for long output times, the increased errors of the MOP-WENO-ACM $k$ scheme are much smaller than those of the WENO-JS and WENO-M schemes. Actually, it is an important advantage of the MOP-WENO-ACM $k$ scheme that can maintain comparably high resolution for long output times. In the next subsection we will demonstrate this again.

Fig. 12 shows the performance of the WENO-JS, WENO-M, MIP-WENO-ACM $k$ and MOP-WENO-ACM $k$ schemes at output time $t=1000$ with a uniform mesh size of $\Delta x=1 / 200$. Clearly, the MIP-WENO-ACM $k$ and MOP-WENO-ACM $k$ schemes give the highest resolution, followed by the WENO-M scheme, whose resolution decreases significantly. The WENO-JS scheme shows the lowest resolution.

Example 4. (Accuracy test with discontinuous initial condition) We consider the SLP modeled by the one-dimensional linear advection equation Eq.14] with the initial condition Eq.15]. In this problem, the CFL number is taken to be 0.1 .

Table 10 shows the $L_{1}, L_{2}, L_{\infty}$ errors and corresponding convergence orders of various considered WENO schemes for this example at output times $t=2$ and $t=2000$. At the short output time $t=2$, we find that: (1) for all considered schemes, the $L_{1}$ and $L_{2}$ orders are approximately 1.0 and 0.4 to 0.5 , respectively, and the $L_{\infty}$ orders are all negative; (2) the MIP-WENO-ACM $k$, WENO-M, WENO-PM6 and WENO-IM $(2,0.1)$ schemes present more accurate results than the MOP-WENO-ACM $k$ and WENO-JS schemes. At the long output time $t=2000$, we find that: (1) for the WENOJS and WENO-M schemes, the $L_{1}, L_{2}$ orders decrease to very small values and even become negative; (2) however, for the MOP-WENO-ACM $k$, MIP-WENO-ACM $k$, WENO-PM6 and WENO-IM $(2,0.1)$ schemes, their $L_{1}$ orders are clearly larger than 1.0 , and their $L_{2}$ orders increase to approximately 0.6 to 0.9 ; (3) for all considered schemes, the $L_{\infty}$ orders are very small and even become negative; (4) in terms of accuracy, on the whole, the MOP-WENO$\mathrm{ACM} k$ scheme produces accurate and comparable results as the other considered mapped WENO schemes except the WENO-M scheme. However, if we take a closer look, we can find that: (1) the resolution of the result computed by the WENO-M scheme is significantly lower than that of the MOP-WENO-ACM $k$ scheme; (2) the WENO-PM6, WENO$\operatorname{IM}(2,0.1)$ and MIP-WENO-ACM $k$ schemes generate spurious oscillations but the MOP-WENO-ACM $k$ scheme does not. More results will be presented carefully to demonstrate this in the following subsection. 
Table 8. Performance of various considered schemes solving $u_{t}+u_{x}=0$ with $u(x, 0)=\sin ^{9}(\pi x), \Delta x=1 / 200$.

\begin{tabular}{|c|c|c|c|c|c|c|c|}
\hline \multirow[b]{2}{*}{ Scheme } & \multirow{2}{*}{$\begin{array}{l}\text { MIP-WENO-ACM } k \\
\text { Errors }\end{array}$} & \multicolumn{2}{|c|}{ MOP-WENO-ACM $k$} & \multicolumn{2}{|l|}{ WENO-JS } & \multicolumn{2}{|l|}{ WENO-M } \\
\hline & & Errors & Increased errors & Errors & Increased errors & Errors & Increased errors \\
\hline \multirow[t]{3}{*}{$\bar{t}=1$} & $8.43356 \mathrm{e}-06$ & $1.73735 \mathrm{e}-05$ & $106.00 \%$ & $3.87826 \mathrm{e}-05$ & $359.86 \%$ & $8.84565 \mathrm{e}-06$ & $4.89 \%$ \\
\hline & $8.20366 \mathrm{e}-06$ & $1.85069 \mathrm{e}-05$ & $125.59 \%$ & $3.62689 \mathrm{e}-05$ & $342.11 \%$ & $8.31248 \mathrm{e}-06$ & $1.33 \%$ \\
\hline & $1.38389 \mathrm{e}-05$ & $6.40784 \mathrm{e}-05$ & $363.03 \%$ & $6.69118 \mathrm{e}-05$ & $383.51 \%$ & $1.38461 \mathrm{e}-05$ & $0.05 \%$ \\
\hline \multirow[t]{3}{*}{$t=10$} & $8.42873 \mathrm{e}-05$ & $1.55900 \mathrm{e}-04$ & $84.96 \%$ & $3.86931 \mathrm{e}-04$ & $359.06 \%$ & $8.90890 \mathrm{e}-05$ & $5.70 \%$ \\
\hline & $8.19107 \mathrm{e}-05$ & $1.63558 \mathrm{e}-04$ & $99.68 \%$ & $3.52611 \mathrm{e}-04$ & $330.48 \%$ & $8.32089 \mathrm{e}-05$ & $1.58 \%$ \\
\hline & $1.38205 \mathrm{e}-04$ & $5.22964 \mathrm{e}-04$ & $278.40 \%$ & $5.36940 \mathrm{e}-04$ & $288.51 \%$ & $1.38348 \mathrm{e}-04$ & $0.10 \%$ \\
\hline \multirow[t]{3}{*}{$t=30$} & $2.52378 \mathrm{e}-04$ & $5.66584 \mathrm{e}-04$ & $124.50 \%$ & $1.17988 \mathrm{e}-03$ & $367.51 \%$ & $2.73430 \mathrm{e}-04$ & $8.34 \%$ \\
\hline & $2.45090 \mathrm{e}-04$ & $6.35696 \mathrm{e}-04$ & $159.37 \%$ & $1.06511 \mathrm{e}-03$ & $334.58 \%$ & $2.51737 \mathrm{e}-04$ & $2.71 \%$ \\
\hline & $4.13398 \mathrm{e}-04$ & $1.99465 \mathrm{e}-03$ & $382.50 \%$ & $1.58134 \mathrm{e}-03$ & $282.52 \%$ & $4.13887 \mathrm{e}-04$ & $0.12 \%$ \\
\hline \multirow[t]{3}{*}{$t=50$} & $4.19825 \mathrm{e}-04$ & $1.09581 \mathrm{e}-03$ & $161.02 \%$ & $2.05488 \mathrm{e}-03$ & $389.46 \%$ & $4.81901 \mathrm{e}-04$ & $14.79 \%$ \\
\hline & $4.07429 \mathrm{e}-04$ & $1.26383 \mathrm{e}-03$ & $210.20 \%$ & $1.84782 \mathrm{e}-03$ & $353.53 \%$ & $4.39983 \mathrm{e}-04$ & $7.99 \%$ \\
\hline & $6.86983 \mathrm{e}-04$ & $3.84112 \mathrm{e}-03$ & $459.13 \%$ & $2.69500 \mathrm{e}-03$ & $292.30 \%$ & $6.87879 \mathrm{e}-04$ & $0.13 \%$ \\
\hline \multirow[t]{3}{*}{$t=100$} & $8.35747 \mathrm{e}-04$ & $2.72470 \mathrm{e}-03$ & $226.02 \%$ & $5.42288 \mathrm{e}-03$ & $548.87 \%$ & $1.29154 \mathrm{e}-03$ & $54.54 \%$ \\
\hline & $8.09679 \mathrm{e}-04$ & $3.23726 \mathrm{e}-03$ & $299.82 \%$ & $5.17716 \mathrm{e}-03$ & $539.41 \%$ & $1.28740 \mathrm{e}-03$ & $59.00 \%$ \\
\hline & $1.36404 \mathrm{e}-03$ & $9.83147 \mathrm{e}-03$ & $620.76 \%$ & $1.20056 \mathrm{e}-02$ & $780.15 \%$ & $3.32665 \mathrm{e}-03$ & $143.88 \%$ \\
\hline \multirow[t]{3}{*}{$t=200$} & $1.65557 \mathrm{e}-03$ & $4.11740 \mathrm{e}-03$ & $148.70 \%$ & $2.35657 \mathrm{e}-02$ & $1323.42 \%$ & $5.74021 \mathrm{e}-03$ & $246.72 \%$ \\
\hline & $1.59929 \mathrm{e}-03$ & $3.71649 \mathrm{e}-03$ & $132.38 \%$ & $2.68753 \mathrm{e}-02$ & $1580.45 \%$ & $7.66721 \mathrm{e}-03$ & $379.41 \%$ \\
\hline & $2.68955 \mathrm{e}-03$ & $6.66166 \mathrm{e}-03$ & $147.69 \%$ & $6.47820 \mathrm{e}-02$ & $2308.66 \%$ & $2.37125 \mathrm{e}-02$ & $781.65 \%$ \\
\hline \multirow[t]{3}{*}{$t=500$} & $3.95849 \mathrm{e}-03$ & $8.34435 \mathrm{e}-03$ & $110.80 \%$ & $1.55650 \mathrm{e}-01$ & 3832.05\% & $4.89290 \mathrm{e}-02$ & $1136.05 \%$ \\
\hline & $3.84802 \mathrm{e}-03$ & $7.96980 \mathrm{e}-03$ & $107.11 \%$ & $1.46859 \mathrm{e}-01$ & $3716.48 \%$ & $6.23842 \mathrm{e}-02$ & $1521.20 \%$ \\
\hline & $6.45564 \mathrm{e}-03$ & $1.83215 \mathrm{e}-02$ & $183.81 \%$ & $2.57663 \mathrm{e}-01$ & $3891.29 \%$ & $1.78294 \mathrm{e}-01$ & $2661.83 \%$ \\
\hline \multirow[t]{3}{*}{$t=1000$} & $7.24723 \mathrm{e}-03$ & $1.54830 \mathrm{e}-02$ & $113.64 \%$ & $2.91359 \mathrm{e}-01$ & $3920.28 \%$ & $1.34933 \mathrm{e}-01$ & $1761.86 \%$ \\
\hline & $7.21626 \mathrm{e}-03$ & $1.50017 \mathrm{e}-02$ & $107.89 \%$ & $2.66692 \mathrm{e}-01$ & $3595.71 \%$ & $1.46524 \mathrm{e}-01$ & $1930.47 \%$ \\
\hline & $1.21593 \mathrm{e}-02$ & $3.16523 \mathrm{e}-02$ & $160.31 \%$ & $4.44664 \mathrm{e}-01$ & $3556.96 \%$ & $3.17199 \mathrm{e}-01$ & $2508.69 \%$ \\
\hline
\end{tabular}

\subsection{Linear advection examples with discontinuities at long output times for comparison}

In this subsection, we will make a further study on calculating linear advection examples with discontinuities at long output times by various considered WENO schemes. The objective is to demonstrate the advantage of the MOPWENO-ACM $k$ scheme that can obtain high resolution and do not generate spurious oscillations, especially for long output time simulations.

The one-dimensional linear advection problem Eq. (14) was used in this study. It was solved with the following two initial conditions.

Case 1. (SLP) The initial condition is given by Eq. 15] in subsection 3.2

Case 2. (BiCWP) The initial condition is given by

$$
u(x, 0)= \begin{cases}0, & x \in[-1.0,-0.8] \cup(-0.2,0.2] \cup(0.8,1.0], \\ 0.5, & x \in(-0.6,-0.4] \cup(0.2,0.4] \cup(0.6,0.8], \\ 1, & x \in(-0.8,-0.6] \cup(-0.4,-0.2] \cup(0.4,0.6],\end{cases}
$$

and the periodic boundary condition is used in the two directions.

Case 1 is the SLP used earlier in this paper. Case 2 consists of several constant states separated by sharp discontinuities at $x= \pm 0.8, \pm 0.6, \pm 0.4, \pm 0.2$. We call Case 2 BiCWP for brevity in the presentation as the profile of the exact solution for this Problem looks like the Breach in City Wall.

We use the uniform mesh of $N=800$ with the output time $t=2000$ and use $N=1600,3200,6400$ with the output time $t=200$ respectively to solve both SLP and BiCWP by all considered WENO schemes. Fig. 13 and Fig. 14 show the comparison of various schemes when $t=2000$ and $N=800$. We can observe that: (1) the MOP-WENO-ACM $k$ scheme provides the numerical results with a significantly higher resolution than those of the WENO-JS and WENOM schemes, and it does not generate spurious oscillations while the WENO-PM6 and MIP-WENO-ACM $k$ schemes do, when solving both SLP and BiCWP; (2) when solving SLP on present computing condition, the WENO-IM(2, 0.1) scheme does not seem to generate spurious oscillations and it gives better resolution than the MOP-WENO-ACM $k$ scheme in most of the region; (3) however, from Fig. 13. b), we observe that the MOP-WENO-ACM $k$ scheme gives a better resolution of the Gaussian than the WENO-IM(2,0.1) scheme, and if we take a closer look, we can see that the WENO-IM(2, 0.1) scheme generate a very slight spurious oscillation near $x=-0.435$ as shown in Fig. 13.c); (4) it is very evident as shown in Fig. 14 that, when solving BiCWP, the WENO-IM(2, 0.1) scheme generates the spurious oscillations.

In Figs. 15, 16, 17 and Figs. 18, 19, 20, we show the comparison of various schemes when $t=200$ and $N=1600,3200,6400$ for SLP and BiCWP, respectively. From these solutions computed with larger grid numbers 
Table 9. Performance of various considered schemes solving $u_{t}+u_{x}=0$ with $u(x, 0)=\sin ^{9}(\pi x), \Delta x=1 / 800$.

\begin{tabular}{|c|c|c|c|c|c|c|c|}
\hline \multirow[b]{2}{*}{ Scheme } & \multirow{2}{*}{$\begin{array}{l}\text { MIP-WENO-ACM } k \\
\text { Errors }\end{array}$} & \multicolumn{2}{|c|}{ MOP-WENO-ACM $k$} & \multicolumn{2}{|l|}{ WENO-JS } & \multicolumn{2}{|l|}{ WENO-M } \\
\hline & & Errors & Increased errors & Errors & Increased errors & Errors & Increased errors \\
\hline \multirow[t]{3}{*}{$\bar{t}=1$} & $8.28796 \mathrm{e}-09$ & $8.33502 \mathrm{e}-09$ & $0.57 \%$ & $4.23539 \mathrm{e}-08$ & $411.03 \%$ & $8.28916 \mathrm{e}-09$ & $0.01 \%$ \\
\hline & $8.06772 \mathrm{e}-09$ & $8.07086 \mathrm{e}-09$ & $0.04 \%$ & $3.78411 \mathrm{e}-08$ & $369.04 \%$ & $8.06776 \mathrm{e}-09$ & $0.00 \%$ \\
\hline & $1.36173 \mathrm{e}-08$ & $1.36173 \mathrm{e}-08$ & $0.00 \%$ & $7.82744 \mathrm{e}-08$ & $474.82 \%$ & $1.36173 \mathrm{e}-08$ & $0.00 \%$ \\
\hline \multirow[t]{3}{*}{$t=10$} & $8.28794 \mathrm{e}-08$ & $8.47930 \mathrm{e}-08$ & $2.31 \%$ & $4.23531 \mathrm{e}-07$ & $411.02 \%$ & $8.28912 \mathrm{e}-08$ & $0.01 \%$ \\
\hline & $8.06769 \mathrm{e}-08$ & $8.11193 \mathrm{e}-08$ & $0.55 \%$ & $3.76804 \mathrm{e}-07$ & $367.05 \%$ & $8.06774 \mathrm{e}-08$ & $0.00 \%$ \\
\hline & $1.36172 \mathrm{e}-07$ & $1.36172 \mathrm{e}-07$ & $0.00 \%$ & $6.95290 \mathrm{e}-07$ & $410.60 \%$ & $1.36173 \mathrm{e}-07$ & $0.00 \%$ \\
\hline \multirow[t]{3}{*}{$t=30$} & $2.48654 \mathrm{e}-07$ & $2.73030 \mathrm{e}-07$ & $9.80 \%$ & $1.27070 \mathrm{e}-06$ & $411.03 \%$ & $2.48687 \mathrm{e}-07$ & $0.01 \%$ \\
\hline & $2.42057 \mathrm{e}-07$ & $2.58215 \mathrm{e}-07$ & $6.68 \%$ & $1.12915 \mathrm{e}-06$ & $366.48 \%$ & $2.42059 \mathrm{e}-07$ & $0.00 \%$ \\
\hline & $4.08575 e-07$ & $5.62109 \mathrm{e}-07$ & $37.58 \%$ & $1.98978 \mathrm{e}-06$ & $387.00 \%$ & $4.08577 \mathrm{e}-07$ & $0.00 \%$ \\
\hline \multirow[t]{3}{*}{$t=50$} & $4.14434 \mathrm{e}-07$ & $5.04422 \mathrm{e}-07$ & $21.71 \%$ & $2.11778 \mathrm{e}-06$ & $411.01 \%$ & $4.14491 \mathrm{e}-07$ & $0.01 \%$ \\
\hline & $4.03460 \mathrm{e}-07$ & $5.02965 \mathrm{e}-07$ & $24.66 \%$ & $1.88080 \mathrm{e}-06$ & $366.17 \%$ & $4.03463 \mathrm{e}-07$ & $0.00 \%$ \\
\hline & $6.81002 \mathrm{e}-07$ & $1.82747 \mathrm{e}-06$ & $168.35 \%$ & $3.25711 \mathrm{e}-06$ & $378.28 \%$ & $6.81005 \mathrm{e}-07$ & $0.00 \%$ \\
\hline \multirow[t]{3}{*}{$t=100$} & $8.28891 \mathrm{e}-07$ & $9.73202 \mathrm{e}-07$ & $17.41 \%$ & $4.74028 \mathrm{e}-06$ & $471.88 \%$ & $8.29015 \mathrm{e}-07$ & $0.01 \%$ \\
\hline & $8.06973 \mathrm{e}-07$ & $9.07161 \mathrm{e}-07$ & $12.42 \%$ & $4.32403 \mathrm{e}-06$ & $435.83 \%$ & $8.06977 \mathrm{e}-07$ & $0.00 \%$ \\
\hline & $1.36206 \mathrm{e}-06$ & $1.79160 \mathrm{e}-06$ & $31.54 \%$ & $1.09481 \mathrm{e}-05$ & $703.79 \%$ & $1.36207 \mathrm{e}-06$ & $0.00 \%$ \\
\hline \multirow[t]{3}{*}{$t=200$} & $1.65782 \mathrm{e}-06$ & $1.78369 \mathrm{e}-06$ & $7.59 \%$ & $7.29285 \mathrm{e}-05$ & $4299.06 \%$ & $2.27991 \mathrm{e}-06$ & $37.52 \%$ \\
\hline & $1.61399 \mathrm{e}-06$ & $1.64768 \mathrm{e}-06$ & $2.09 \%$ & $1.60499 \mathrm{e}-04$ & $9844.24 \%$ & $2.59031 \mathrm{e}-06$ & $60.49 \%$ \\
\hline & $2.72415 \mathrm{e}-06$ & $2.72415 \mathrm{e}-06$ & $0.00 \%$ & $9.51604 \mathrm{e}-04$ & $34832.14 \%$ & $1.22731 \mathrm{e}-05$ & 350.53\% \\
\hline \multirow[t]{3}{*}{$t=500$} & $4.14451 \mathrm{e}-06$ & $4.84739 \mathrm{e}-06$ & $16.96 \%$ & $3.11698 \mathrm{e}-02$ & $751974.43 \%$ & $1.41413 \mathrm{e}-03$ & $34020.56 \%$ \\
\hline & $4.03492 \mathrm{e}-06$ & $4.47345 \mathrm{e}-06$ & $10.87 \%$ & $4.08456 \mathrm{e}-02$ & $1012202.60 \%$ & $3.28891 \mathrm{e}-03$ & $81411.16 \%$ \\
\hline & $6.81018 \mathrm{e}-06$ & $8.79296 \mathrm{e}-06$ & $29.11 \%$ & $8.63989 \mathrm{e}-02$ & $1268572.78 \%$ & $1.90785 \mathrm{e}-02$ & $280046.78 \%$ \\
\hline \multirow[t]{3}{*}{$t=1000$} & $8.28868 \mathrm{e}-06$ & $8.61232 \mathrm{e}-06$ & $3.90 \%$ & $1.01278 \mathrm{e}-01$ & $1221783.34 \%$ & $1.83325 \mathrm{e}-02$ & $221075.14 \%$ \\
\hline & $8.06938 \mathrm{e}-06$ & $8.14436 \mathrm{e}-06$ & $0.93 \%$ & $1.13316 \mathrm{e}-01$ & $1404171.46 \%$ & $3.30753 \mathrm{e}-02$ & $409786.51 \%$ \\
\hline & $1.36194 \mathrm{e}-05$ & $1.36194 \mathrm{e}-05$ & $0.00 \%$ & $2.13485 \mathrm{e}-01$ & $1567406.64 \%$ & $1.38215 \mathrm{e}-01$ & $1014739.13 \%$ \\
\hline
\end{tabular}

Table 10. Convergence properties of various considered schemes solving $u_{t}+u_{x}=0$ with initial condition Eq. (15).

\begin{tabular}{|c|c|c|c|c|c|c|}
\hline \multirow[b]{2}{*}{$N$} & \multicolumn{3}{|l|}{$t=2$} & \multicolumn{3}{|l|}{$t=2000$} \\
\hline & 200 & 400 & 800 & 200 & 400 & 800 \\
\hline \multirow[t]{3}{*}{ WENO-JS } & $6.30497 \mathrm{e}-02(-)$ & $2.81654 \mathrm{e}-02(1.2103)$ & $1.41364 \mathrm{e}-02(0.9945)$ & $6.12899 \mathrm{e}-01(-)$ & $5.99215 \mathrm{e}-01(0.0326)$ & $5.50158 \mathrm{e}-01(0.1232)$ \\
\hline & $1.08621 \mathrm{e}-01(-)$ & $7.71111 \mathrm{e}-02(0.4943)$ & $5.69922 \mathrm{e}-02(0.4362)$ & $5.08726 \mathrm{e}-01(-)$ & $5.01160 \mathrm{e}-01(0.0216)$ & $4.67585 \mathrm{e}-01(0.1000)$ \\
\hline & $4.09733 \mathrm{e}-01(-)$ & $4.19594 \mathrm{e}-01(-0.0343)$ & $4.28463 \mathrm{e}-01(-0.0302)$ & $7.99265 \mathrm{e}-01(-)$ & $8.20493 \mathrm{e}-01(-0.0378)$ & $8.14650 \mathrm{e}-01(0.0103)$ \\
\hline \multirow[t]{3}{*}{ WENO-M } & $4.77201 \mathrm{e}-02(-)$ & $2.23407 \mathrm{e}-02(1.0949)$ & $1.11758 \mathrm{e}-02(0.9993)$ & $3.81597 \mathrm{e}-01(-)$ & $3.25323 \mathrm{e}-01(0.2302)$ & $3.48528 \mathrm{e}-01(-0.0994)$ \\
\hline & $9.53073 \mathrm{e}-02(-)$ & $6.91333 \mathrm{e}-02(0.4632)$ & $5.09232 \mathrm{e}-02(0.4411)$ & $3.59205 \mathrm{e}-01(-)$ & $3.12970 \mathrm{e}-01(0.1988)$ & $3.24373 \mathrm{e}-01(-0.0516)$ \\
\hline & $3.94243 \mathrm{e}-01(-)$ & $4.05856 \mathrm{e}-01(-0.0419)$ & $4.16937 \mathrm{e}-01(-0.0389)$ & $6.89414 \mathrm{e}-01(-)$ & $6.75473 \mathrm{e}-01(0.0295)$ & $6.25645 \mathrm{e}-01(0.1106)$ \\
\hline \multirow[t]{3}{*}{ WENO-PM6 } & $4.66681 \mathrm{e}-02(-)$ & $2.13883 \mathrm{e}-02(1.1256)$ & $1.06477 \mathrm{e}-02(1.0063)$ & $2.17323 \mathrm{e}-01(-)$ & $1.05197 \mathrm{e}-01(1.0467)$ & $4.47030 \mathrm{e}-02(1.2347)$ \\
\hline & $9.45566 \mathrm{e}-02(-)$ & $6.82948 \mathrm{e}-02(0.4694)$ & $5.03724 \mathrm{e}-02(0.4391)$ & $2.28655 \mathrm{e}-01(-)$ & $1.47518 \mathrm{e}-01(0.6323)$ & $9.34250 \mathrm{e}-02(0.6590)$ \\
\hline & $3.96866 \mathrm{e}-01(-)$ & $4.06118 \mathrm{e}-01(-0.0332)$ & $4.15277 \mathrm{e}-01(-0.0322)$ & $5.63042 \mathrm{e}-01(-)$ & $5.04977 \mathrm{e}-01(0.1570)$ & $4.71368 \mathrm{e}-01(0.0994)$ \\
\hline \multirow[t]{3}{*}{ WENO-IM $(2,0.1)$} & $4.40293 \mathrm{e}-02(-)$ & $2.02331 \mathrm{e}-02(1.1217)$ & $1.01805 \mathrm{e}-02(0.9909)$ & $2.17411 \mathrm{e}-01(-)$ & $1.12590 \mathrm{e}-01(0.9493)$ & $5.18367 \mathrm{e}-02(1.1190)$ \\
\hline & $9.19118 \mathrm{e}-02(-)$ & $6.68479 \mathrm{e}-02(0.4594)$ & $4.95333 \mathrm{e}-02(0.4325)$ & $2.30000 \mathrm{e}-01(-)$ & $1.64458 \mathrm{e}-01(0.4839)$ & $9.98968 \mathrm{e}-02(0.7192)$ \\
\hline & $3.86789 \mathrm{e}-01(-)$ & $3.98769 \mathrm{e}-01(-0.0441)$ & $4.09515 \mathrm{e}-01(-0.0383)$ & $5.69864 \mathrm{e}-01(-)$ & $4.82180 \mathrm{e}-01(0.2410)$ & $4.73102 \mathrm{e}-01(0.02784)$ \\
\hline \multirow[t]{3}{*}{ MIP-WENO-ACM $k$} & $4.45059 \mathrm{e}-02(-)$ & $2.03667 \mathrm{e}-02(1.1278)$ & $1.02183 \mathrm{e}-02(0.9951)$ & $2.21312 \mathrm{e}-01(-)$ & $1.10365 \mathrm{e}-01(1.0038)$ & $4.76589 \mathrm{e}-02(1.2115)$ \\
\hline & $9.24356 \mathrm{e}-02(-)$ & $6.70230 \mathrm{e}-02(0.4638)$ & $4.96081 \mathrm{e}-02(0.4341)$ & $2.28433 \mathrm{e}-01(-)$ & $1.48498 \mathrm{e}-01(0.6213)$ & $9.40843 \mathrm{e}-02(0.6584)$ \\
\hline & $3.92505 \mathrm{e}-01(-)$ & $4.04024 \mathrm{e}-01(-0.0417)$ & $4.13511 \mathrm{e}-01(-0.0335)$ & $5.36242 \mathrm{e}-01(-)$ & $5.13503 \mathrm{e}-01(0.0625)$ & $5.15898 \mathrm{e}-01(-0.0067)$ \\
\hline \multirow[t]{3}{*}{ MOP-WENO-ACM $k$} & $5.56533 \mathrm{e}-02(-)$ & $2.79028 \mathrm{e}-02(0.9961)$ & $1.43891 \mathrm{e}-02(0.9554)$ & $3.83033 \mathrm{e}-01(-)$ & $1.77114 \mathrm{e}-01(1.1128)$ & $6.70535 \mathrm{e}-02(1.4013)$ \\
\hline & $9.94223 \mathrm{e}-02(-)$ & $7.33101 \mathrm{e}-02(0.4396)$ & $5.51602 \mathrm{e}-02(0.4104)$ & $3.46814 \mathrm{e}-01(-)$ & $1.87369 \mathrm{e}-01(0.8883)$ & $1.09368 \mathrm{e}-01(0.7767)$ \\
\hline & $4.03765 \mathrm{e}-01(-)$ & $4.48412 \mathrm{e}-01(-0.1513)$ & $4.67036 \mathrm{e}-01(-0.0587)$ & $7.18464 \mathrm{e}-01(-)$ & $5.05980 \mathrm{e}-01(0.5058)$ & $4.80890 \mathrm{e}-01(0.0734)$ \\
\hline
\end{tabular}

and a shortened but still long output time, we observe that, as the grid number increases: (1) firstly, the WENO-IM(2, $0.1)$ scheme generates spurious oscillations but the MOP-WENO-ACM $k$ scheme does not while provides an improved resolution when solving SLP; (2) although the resolutions of the results computed by the WENO-JS and WENOM schemes are significantly improved for both SLP and BiCWP, the MOP-WENO-ACM $k$ scheme still evidently provides better resolutions than those of these two schemes; (3) the spurious oscillations generated by the WENOPM6, WENO-IM(2,0.1) and MIP-WENO-ACM $k$ schemes appear to be more evident and more intense when the grid number gets larger, while the MOP-WENO-ACM $k$ scheme can still prevent generating spurious oscillations but obtain great improvement of the resolution, when solving both SLP and BiCWP.

As examples, in Fig. 21, we present the non-OP points in the numerical solutions of SLP with a uniform mesh size of $N=3200$ computed by the WENO-M and MOP-WENO-ACM $k$ schemes, and the non-OP points in the numerical solutions of BiCWP with a uniform mesh size of $N=6400$ computed by the MIP-WENO-ACM $k$ and MOP-WENO$\mathrm{ACM} k$ schemes. We can see that there are a great many non-OP points in the solutions of the SLP computed by the WENO-M scheme and in the solutions of the BiCWP computed by the MIP-WENO-ACM $k$ scheme, while the numbers of the non-OP points in the solutions of these two cases computed by the MOP-WENO-ACM $k$ scheme 
(a)

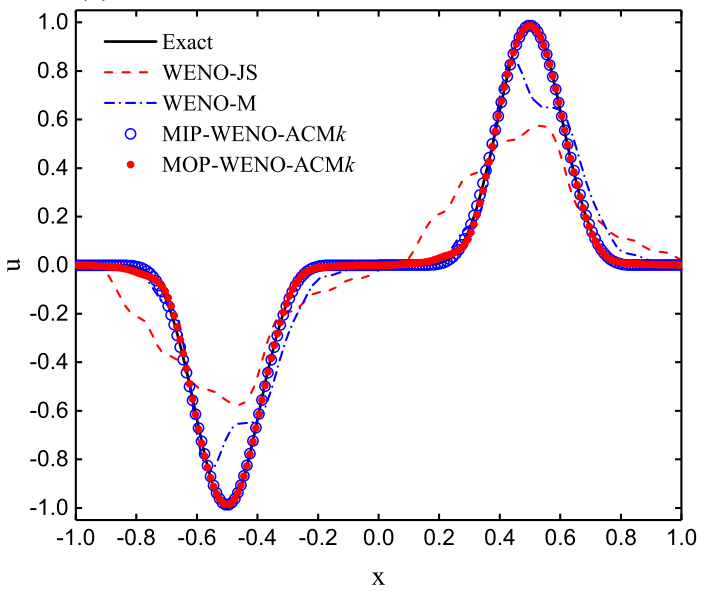

(b)

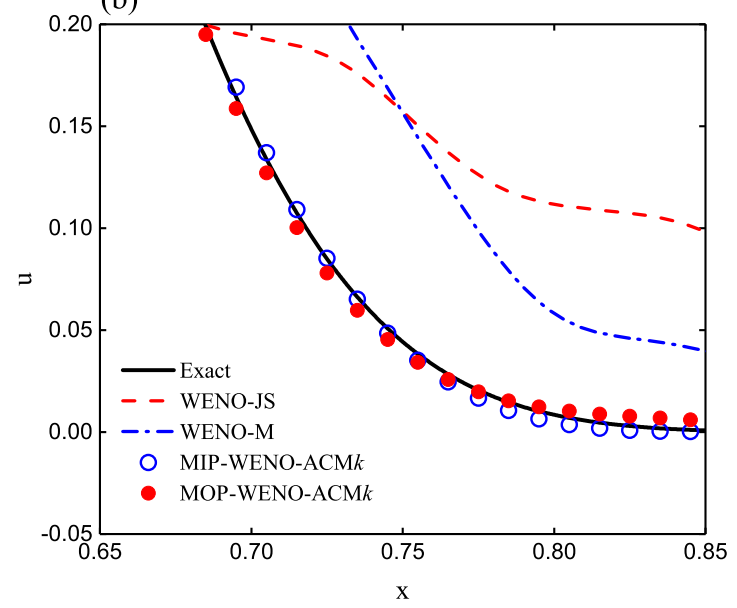

Fig. 12. Performance of the MOP-WENO-ACM $k$, MIP-WNEO-ACM $k$, WENO-JS and WENO-M schemes for Example 3 at output time $t=1000$ with a uniform mesh size of $\Delta x=1 / 200$.

are zero. Actually, there are many non-OP points for all considered mapped schemes whose mapping functions are non-OP, like the WENO-M, WENO-PM6, WENO-IM $(2,0.1))$ and MIP-WENO-ACM $k$ schemes. As expected, there are no non-OP points for the MOP-WENO-ACM $k$ and WENO-JS schemes for all computing cases here. We do not show the results of the non-OP points for all computing cases here just for the simplicity of illustration. It should be noted that the WENO-JS scheme could be treated as a mapped WENO scheme whose mapping function is an identical mapping, that is, $\left(g^{\mathrm{JS}}\right)_{s}(\omega)=\omega, s=0,1,2$, and it is trivial to verify that the set of mapping functions $\left(g^{\mathrm{JS}}\right)_{s}(\omega), s=0,1,2$ is $O P$ while its optimal weight intervals are zero.

Thus, to sum up, we can conclude that a set of mapping functions which is $O P$ can help to improve the resolution of the corresponding mapped WENO scheme and prevent it from generating spurious oscillations in the simulation of problems with discontinuities, especially for long output times. And in the upcoming follow-up study of this article, we will provide more examples and evidence to further verify this conclusion.
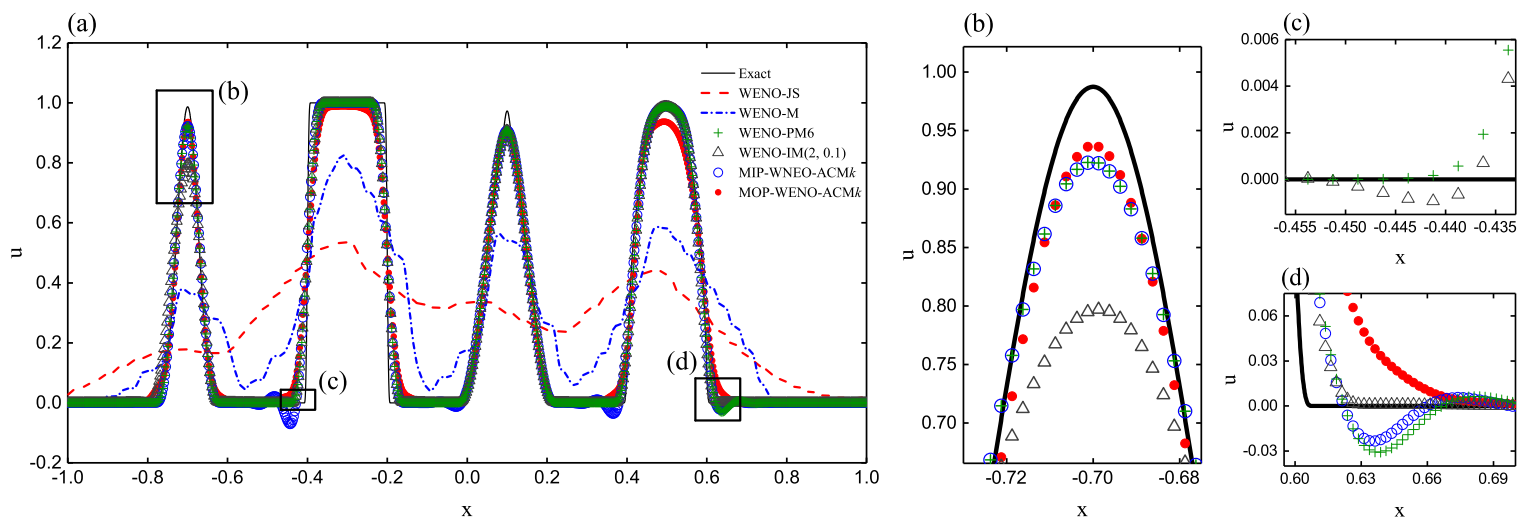

Fig. 13. Performance of the fifth-order MOP-WENO-ACM $k$, MIP-WNEO-ACM $k$, WENO-JS, WENO-M, WENOPM6 and WENO-IM(2,0.1) schemes for the SLP with $N=800$ at output time $t=2000$.

\subsection{Two-dimensional Euler system}

In this subsection, we solve the two-dimensional Euler system of gas dynamics. We consider the numerical solutions of the 2D Riemann problem [28, 27, 18] and the shock-vortex interaction problem [4, 23, 25]. The two- 

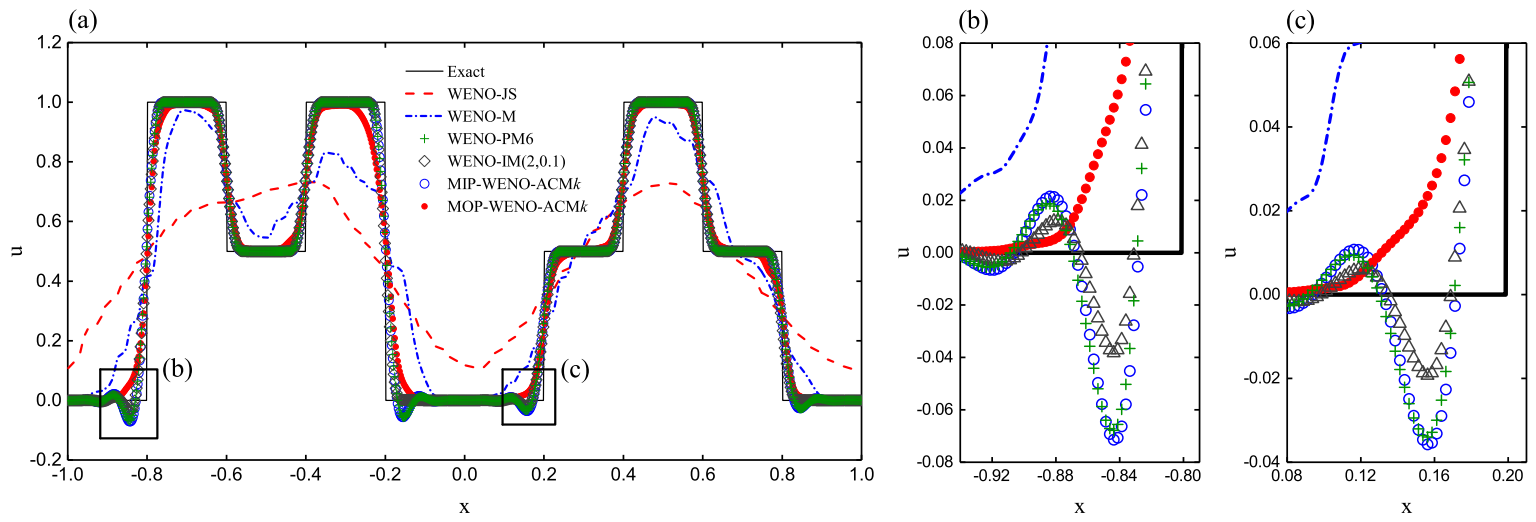

Fig. 14. Performance of the fifth-order MOP-WENO-ACM $k$, MIP-WENO-ACM $k$, WENO-JS, WENO-M, WENOPM6 and WENO-IM $(2,0.1)$ schemes for the BiCWP with $N=800$ at long output time $t=2000$.
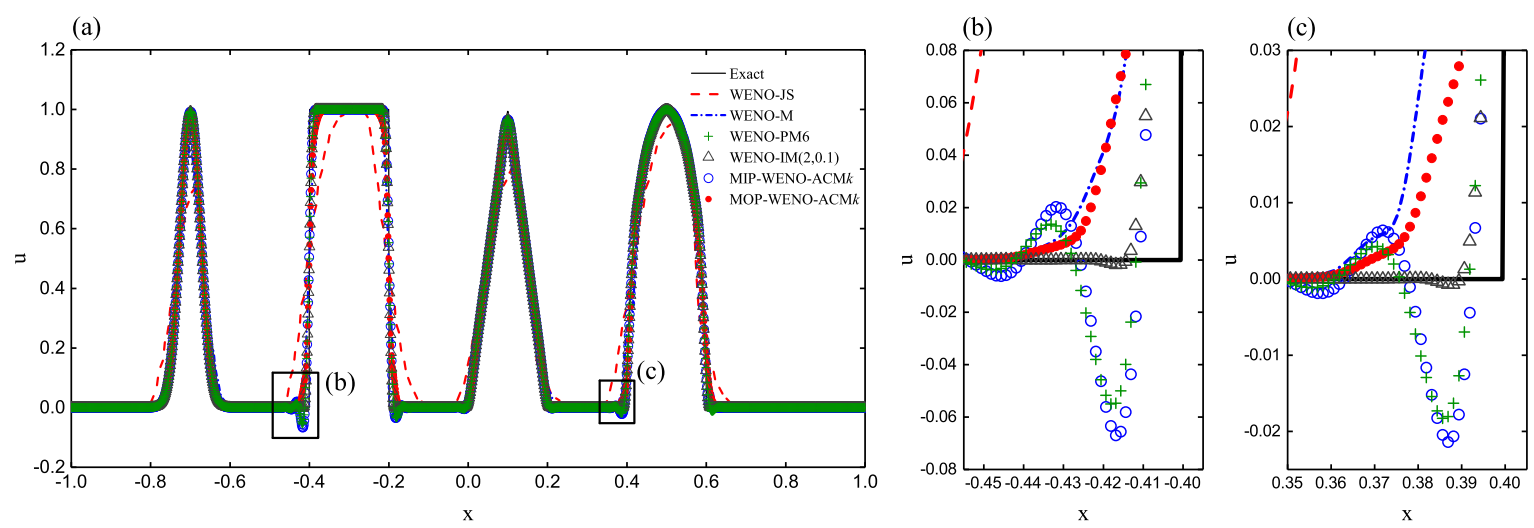

Fig. 15. Performance of the fifth-order MOP-WENO-ACM $k$, MIP-WENO-ACM $k$, WENO-JS, WENO-M, WENOPM6 and WENO-IM $(2,0.1)$ schemes for the SLP with $N=1600$ at long output time $t=200$.

dimensional Euler system is given by the following strong conservation form

$$
\mathbf{U}_{t}+\mathbf{F}(\mathbf{U})_{x}+\mathbf{G}(\mathbf{U})_{y}=0,
$$

where $\mathbf{U}=(\rho, \rho u, \rho v, E)^{\mathrm{T}}, \mathbf{F}(\mathbf{U})=\left(\rho u, \rho u^{2}+p, \rho u v, u(E+p)\right)^{\mathrm{T}}, \mathbf{G}(\mathbf{U})=\left(\rho v, \rho v u, \rho v^{2}+p, v(E+p)\right)^{\mathrm{T}}$, and $\rho, u, v, p$ and $E$ are the density, component of velocity in the $x$ and $y$ coordinate directions, pressure and total energy, respectively. The relation of the pressure and the total energy, the component of velocity in the $x$ and $y$ coordinate directions is defined by the equation of state for an ideal polytropic gas, taking the form

$$
p=(\gamma-1)\left(E-\frac{1}{2} \rho\left(u^{2}+v^{2}\right)\right),
$$

where $\gamma$ is the ratio of specific heats and we choose $\gamma=1.4$ here. In all numerical examples of this subsection, the CFL number is set to be 0.5 .

Example 5. (2D Riemann problem) The series of 2D Riemann problems proposed in [28, 27] has become favorable cases to test the resolution of numerical methods [18, 21, 24]. It is calculated over a unit square domain $[0,1] \times[0,1]$, initially involves the constant states of flow variables over each quadrant which is got by dividing the computational domain using lines $x=x_{0}$ and $y=y_{0}$. Configuration 4 in [18] is taken here for the test. The initial condition of this configuration is given by 

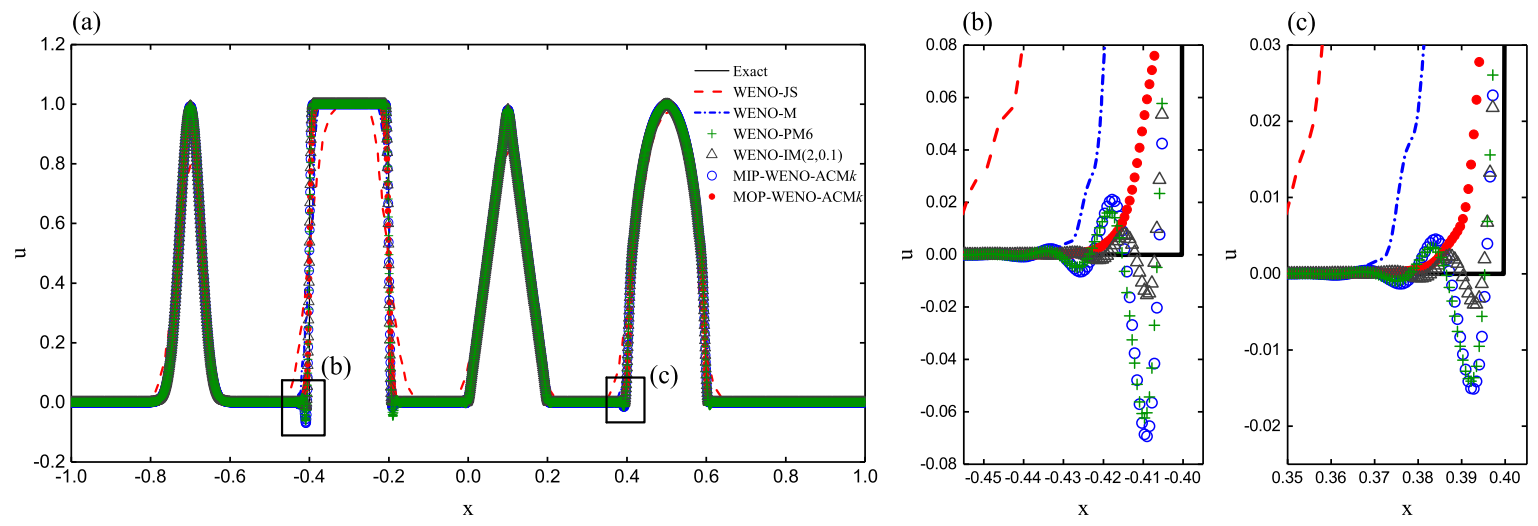

Fig. 16. Performance of the fifth-order MOP-WENO-ACM $k$, MIP-WENO-ACM $k$, WENO-JS, WENO-M, WENOPM6 and WENO-IM $(2,0.1)$ schemes for the SLP with $N=3200$ at long output time $t=200$.
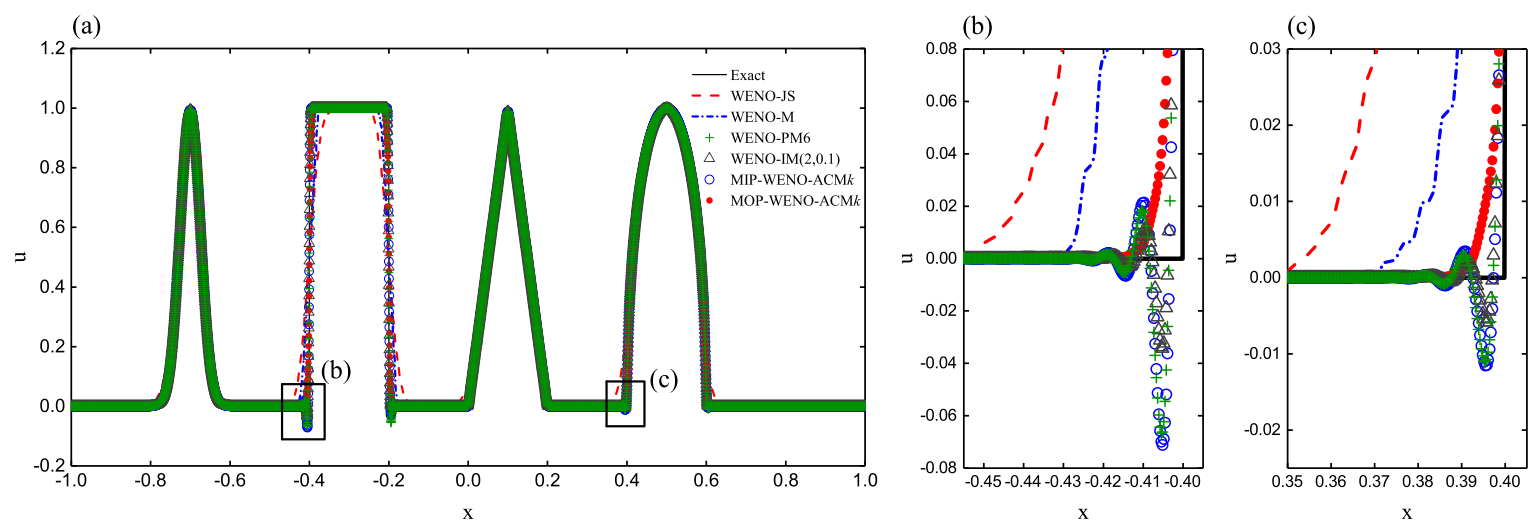

Fig. 17. Performance of the fifth-order MOP-WENO-ACM $k$, MIP-WENO-ACM $k$, WENO-JS, WENO-M, WENOPM6 and WENO-IM $(2,0.1)$ schemes for the SLP with $N=6400$ at long output time $t=200$.

$$
(\rho, u, v, p)(x, y, 0)= \begin{cases}(1.1,0.0,0.0,1.1), & 0.5 \leq x \leq 1.0,0.5 \leq y \leq 1.0, \\ (0.5065,0.8939,0.0,0.35), & 0.0 \leq x \leq 0.5,0.5 \leq y \leq 1.0, \\ (1.1,0.8939,0.8939,1.1), & 0.0 \leq x \leq 0.5,0.0 \leq y \leq 0.5, \\ (0.5065,0.0,0.8939,0.35), & 0.5 \leq x \leq 1.0,0.0 \leq y \leq 0.5 .\end{cases}
$$

The transmission boundary condition is used on all boundaries. The numerical solutions are calculated using considered WENO schemes on $800 \times 800$ cells, and the computations proceed to $t=0.25$.

In Fig. 22, we have shown the numerical results of density obtained by using the WENO-JS, WENO-M, WENOPM6, WENO-IM(2, 0.1), MIP-WENO-ACM $k$ and MOP-WENO-ACM $k$ schemes. We can see that all considered schemes can capture the main structure of the solution. However, we can also observe that there are obvious numerical oscillations (as marked by the pink boxes), which are unfavorable for the fidelity of the results, in the solutions of the WENO-M, WENO-PM6, WENO-IM $(2,0.1)$ and MIP-WENO-ACM $k$ schemes. These numerical oscillations can be seen more clearly from the cross-sectional slices of density profile along the plane $y=0.5$ as presented in Fig. 23, where the reference solution is obtained by using the WENO-JS scheme with a uniform mesh size of $3000 \times 3000$. Noticeably, there are almost no numerical oscillations in the solutions of the MOP-WENO-ACM $k$ and WENO-JS schemes, and this should be an advantage of the mapped WENO schemes whose mapping functions are $O P$.

Example 6. (Shock-vortex interaction) We solve the shock-vortex interaction problem [4, 23, 25] that consists of the interaction of a left moving shock wave with a right moving vortex. The initial condition is given by 

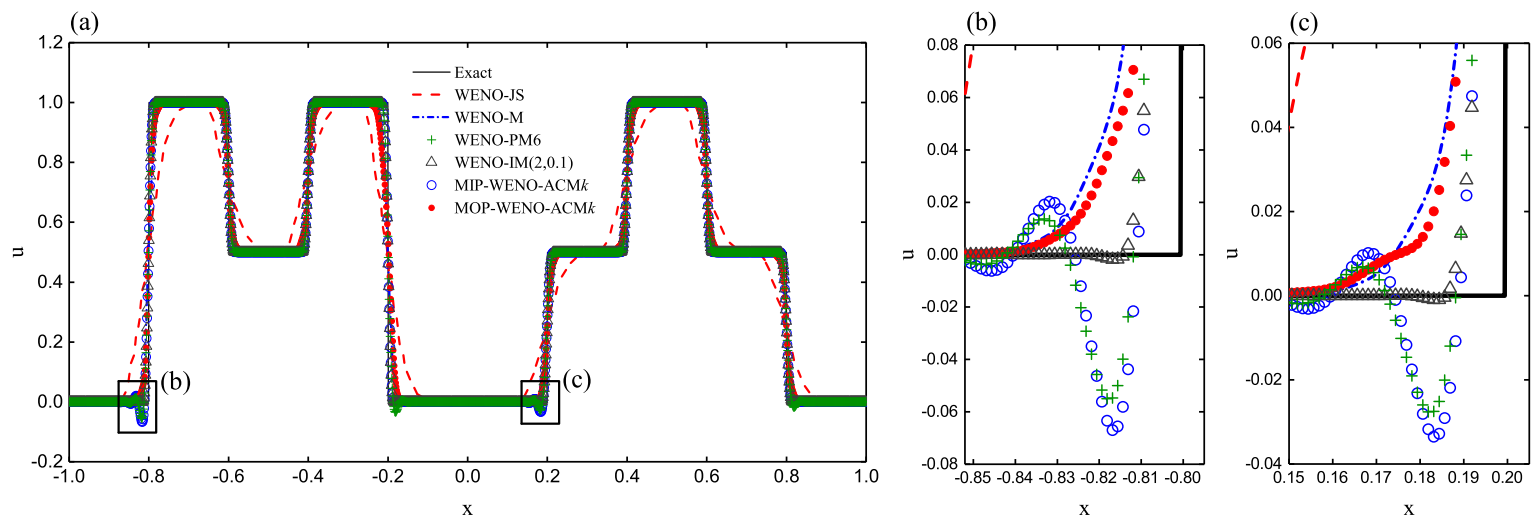

Fig. 18. Performance of the fifth-order MOP-WENO-ACM $k$, MIP-WENO-ACM $k$, WENO-JS, WENO-M, WENOPM6 and WENO-IM $(2,0.1)$ schemes for the BiCWP with $N=1600$ at long output time $t=200$.
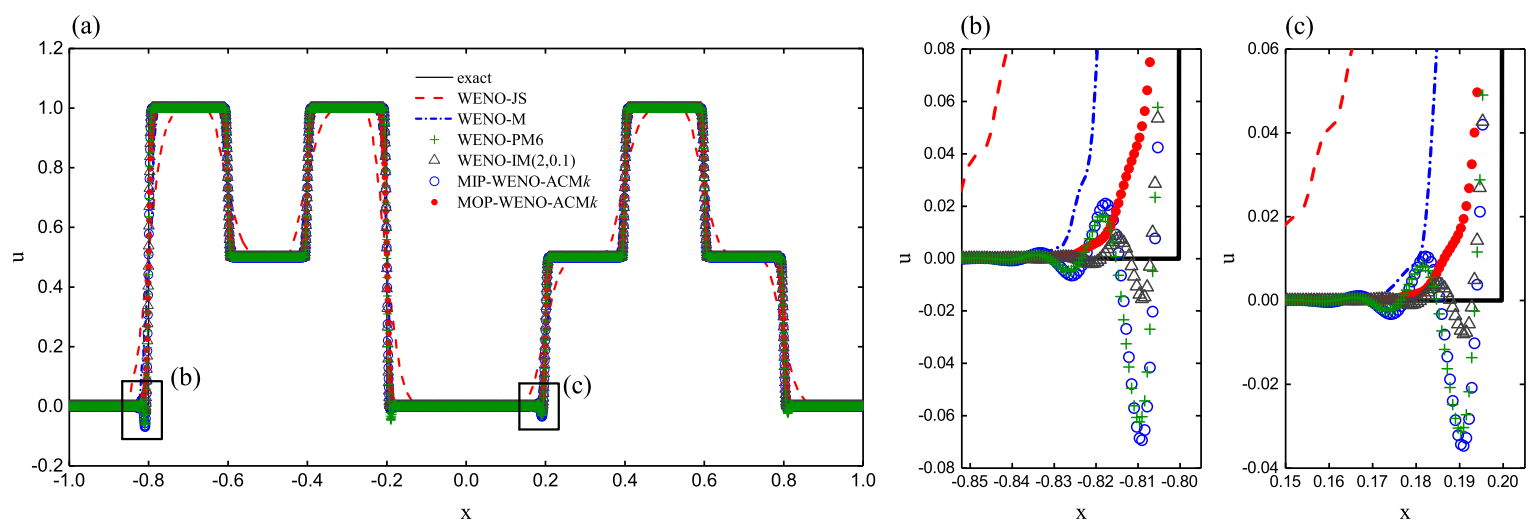

Fig. 19. Performance of the fifth-order MOP-WENO-ACM $k$, MIP-WENO-ACM $k$, WENO-JS, WENO-M, WENOPM6 and WENO-IM $(2,0.1)$ schemes for the BiCWP with $N=3200$ at long output time $t=200$.

$$
(\rho, u, v, p)(x, y, 0)= \begin{cases}\mathbf{U}_{\mathrm{L}}, & x<0.5, \\ \mathbf{U}_{\mathrm{R}}, & x \geq 0.5,\end{cases}
$$

where the left state is taken as $\mathbf{U}_{\mathrm{L}}=\left(\rho_{\mathrm{L}}, u_{\mathrm{L}}, v_{\mathrm{L}}, p_{\mathrm{L}}\right)=(1, \sqrt{\gamma}, 0,1)$, and the right state $\mathbf{U}_{\mathrm{R}}=\left(\rho_{\mathrm{R}}, u_{\mathrm{R}}, v_{\mathrm{R}}, p_{\mathrm{R}}\right)$ is given by

$$
\begin{aligned}
& p_{\mathrm{R}}=1.3, \rho_{\mathrm{R}}=\rho_{\mathrm{L}}\left(\frac{\gamma-1+(\gamma+1) p_{\mathrm{R}}}{\gamma+1+(\gamma-1) p_{\mathrm{R}}}\right) \\
& u_{\mathrm{R}}=u_{\mathrm{L}}\left(\frac{1-p_{\mathrm{R}}}{\sqrt{\gamma-1+p_{\mathrm{R}}(\gamma+1)}}\right), \nu_{\mathrm{R}}=0 .
\end{aligned}
$$

A vortex given by the following perturbations is superimposed onto the left state $\mathbf{U}_{\mathrm{L}}$,

$$
\delta \rho=\frac{\rho_{\mathrm{L}}^{2}}{(\gamma-1) p_{\mathrm{L}}} \delta T, \delta u=\epsilon \frac{y-y_{\mathrm{c}}}{r_{\mathrm{c}}} \mathrm{e}^{\alpha\left(1-r^{2}\right)}, \delta v=-\epsilon \frac{x-x_{\mathrm{c}}}{r_{\mathrm{c}}} \mathrm{e}^{\alpha\left(1-r^{2}\right)}, \delta p=\frac{\gamma \rho_{\mathrm{L}}^{2}}{(\gamma-1) \rho_{\mathrm{L}}} \delta T,
$$

where $\epsilon=0.3, r_{\mathrm{c}}=0.05, \alpha=0.204, x_{\mathrm{c}}=0.25, y_{\mathrm{c}}=0.5, r=\sqrt{\left(\left(x-x_{\mathrm{c}}\right)^{2}+\left(y-y_{\mathrm{c}}\right)^{2}\right) / r_{\mathrm{c}}^{2}}, \delta T=-(\gamma-1) \epsilon^{2} \mathrm{e}^{2 \alpha\left(1-r^{2}\right)} /(4 \alpha \gamma)$. The transmissive boundary condition is used on all boundaries.

The problem has been calculated by the considered WENO schemes with a uniform mesh size of $800 \times 800$ and the output time is taken as $t=0.35$. The final structures of the shock and vortex in the density profile have been shown in Fig. 24. It is observed that all the considered WENO schemes perform well in capturing the main structure 

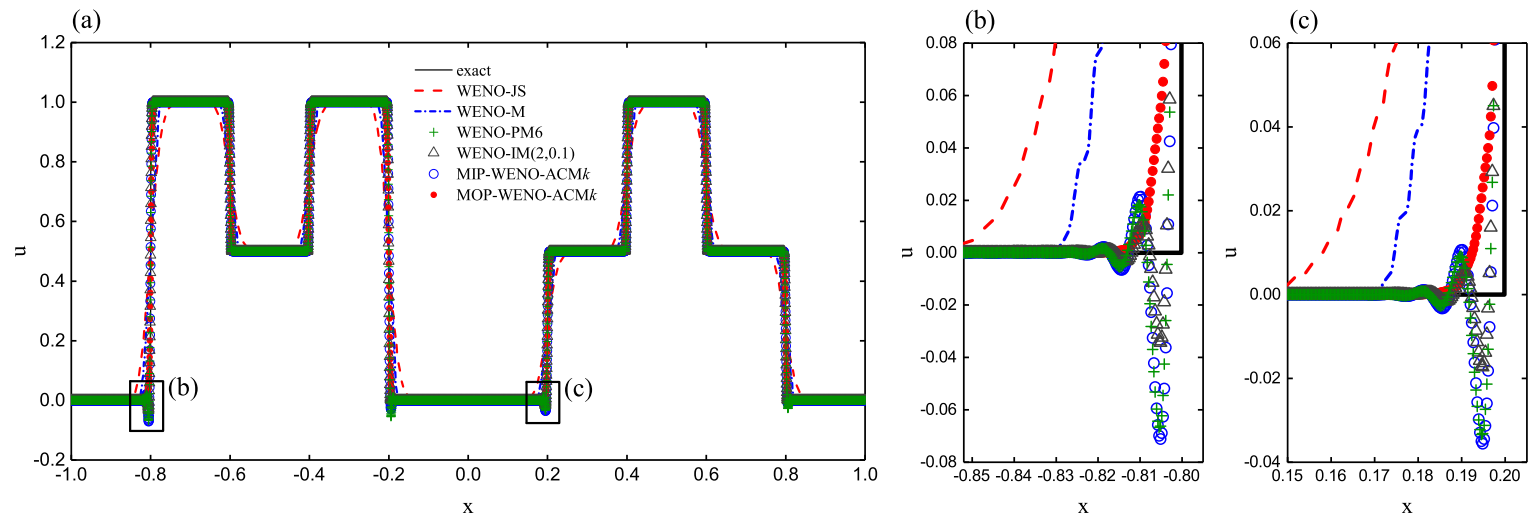

Fig. 20. Performance of the fifth-order MOP-WENO-ACM $k$, MIP-WENO-ACM $k$, WENO-JS, WENO-M, WENOPM6 and WENO-IM $(2,0.1)$ schemes for the BiCWP with $N=6400$ at long output time $t=200$.

of the shock and vortex after the interaction. We can see that there are clear numerical oscillations in the solutions of the WENO-IM $(2,0.1)$ and MIP-WENO-ACM $k$ schemes, and the numerical oscillations can also be observed in the solutions of the WENO-M and WENO-PM6 schemes although they are not so severe as those of the WENO-IM(2, 0.1) and MIP-WENO-ACM $k$ schemes. However, in the solutions of the MOP-WENO-ACM $k$ and WENO-JS schemes, we almost did not find the numerical oscillations. To further demonstrate this, we have plotted the cross sectional slices of density profile along the plane $y=0.65$ in Fig. 25. The reference solution is obtained using the WENO-JS scheme with a uniform mesh size of $1600 \times 1600$. It is evident that the MIP-WENO-ACM $k$ scheme produces the numerical oscillations with the biggest amplitudes followed by those of the WENO-IM(2, 0.1) scheme. The WENO-PM6 and WENO-M schemes also generate clear numerical oscillations with the amplitudes slightly smaller than that of the WENO-IM(2, 0.1) scheme. Obviously, the solutions of the MOP-WENO-ACM $k$ and WENO-JS schemes almost generate no numerical oscillations or only generate some imperceptible numerical oscillations, and their solutions are most close to the reference solution. Again, we argue that this should be an advantage of the mapped WENO schemes whose mapping functions are $O P$.

\section{Conclusions}

This paper has proposed a new mapped weighted essentially non-oscillatory scheme named as MOP-WENO$\operatorname{ACM} k$. The motivation to design this new scheme is that: (1) the WENO-JS and WENO-M schemes generate numerical solutions with very low resolutions when solving hyperbolic problems with discontinuities for long output times; (2) although various existing improved mapped WENO schemes can successfully address the drawback above, as far as we know, almost all of them introduce unfavorable spurious oscillations as the non-OP mapping process occurs in their mappings. By introducing a set of mapping functions that is order-preserving $(O P)$, the MOP-WENO-ACM $k$ scheme can prevent the non-OP mapping process, which should be the essential cause of the spurious oscillation generation and potential loss of accuracy. Therefore, the MOP-WENO-ACM $k$ scheme has a significant advantage in that it not only can obtain comparable high resolutions but also can prevent generating spurious oscillations, when solving problems with discontinuities, especially for long output times. Numerical experiments have shown that the proposed scheme yields lower dissipation and higher resolution near discontinuities than the WENO-JS and WENOM schemes, especially for long output times, and it enjoys better robustness than the WENO-PM6, WENO-IM $(2,0.1)$ and MIP-WENO-ACM $k$ schemes. 

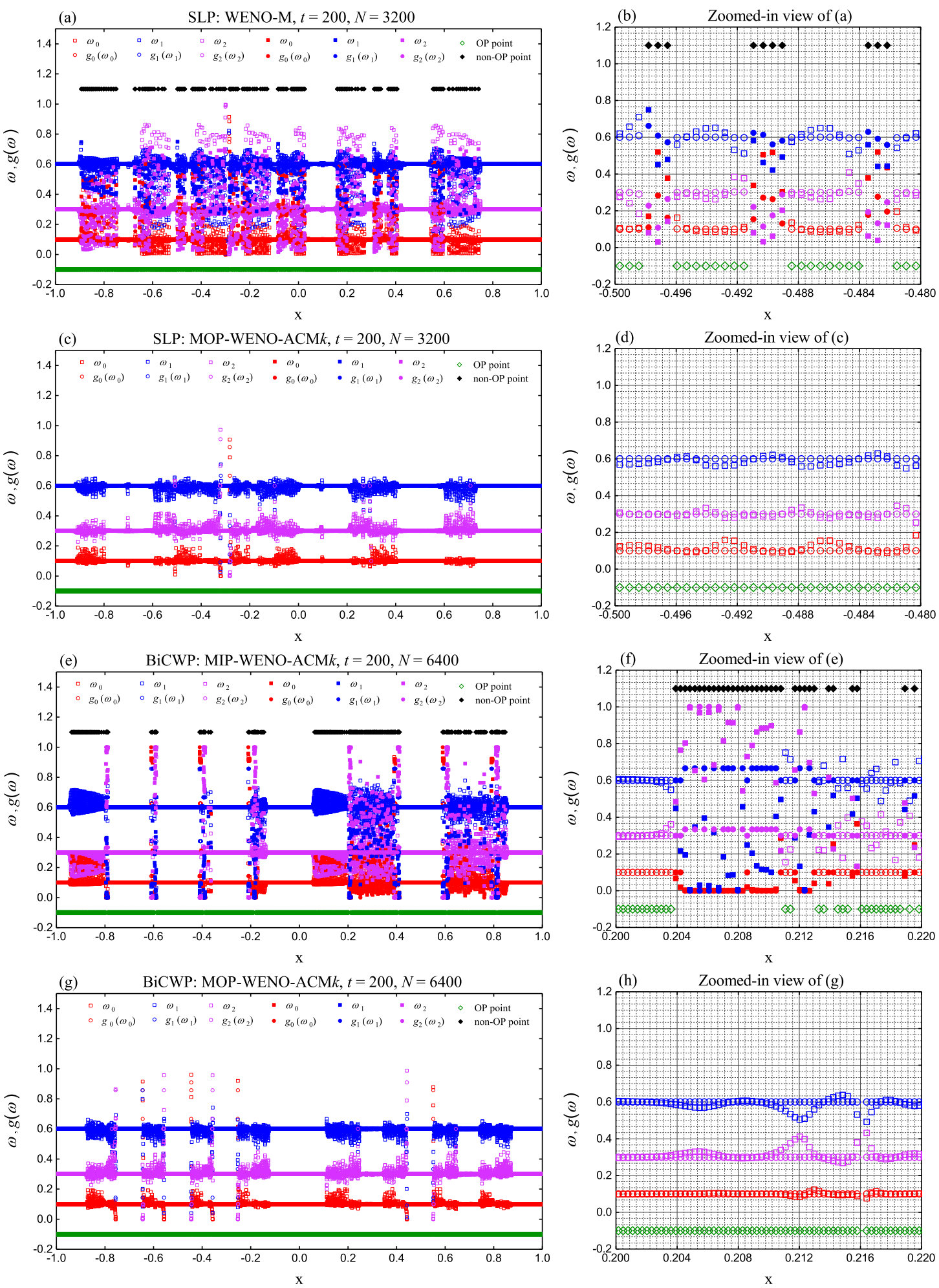

Fig. 21. The non-OP points in the numerical solutions of SLP computed by the WENO-M and MOP-WENO-ACM $k$ schemes with $N=3200, t=200$, and the non-OP points in the numerical solutions of BiCWP computed by the MIP-WENO-ACM $k$ and MOP-WENO-ACM $k$ schemes with $N=6400, t=200$. 

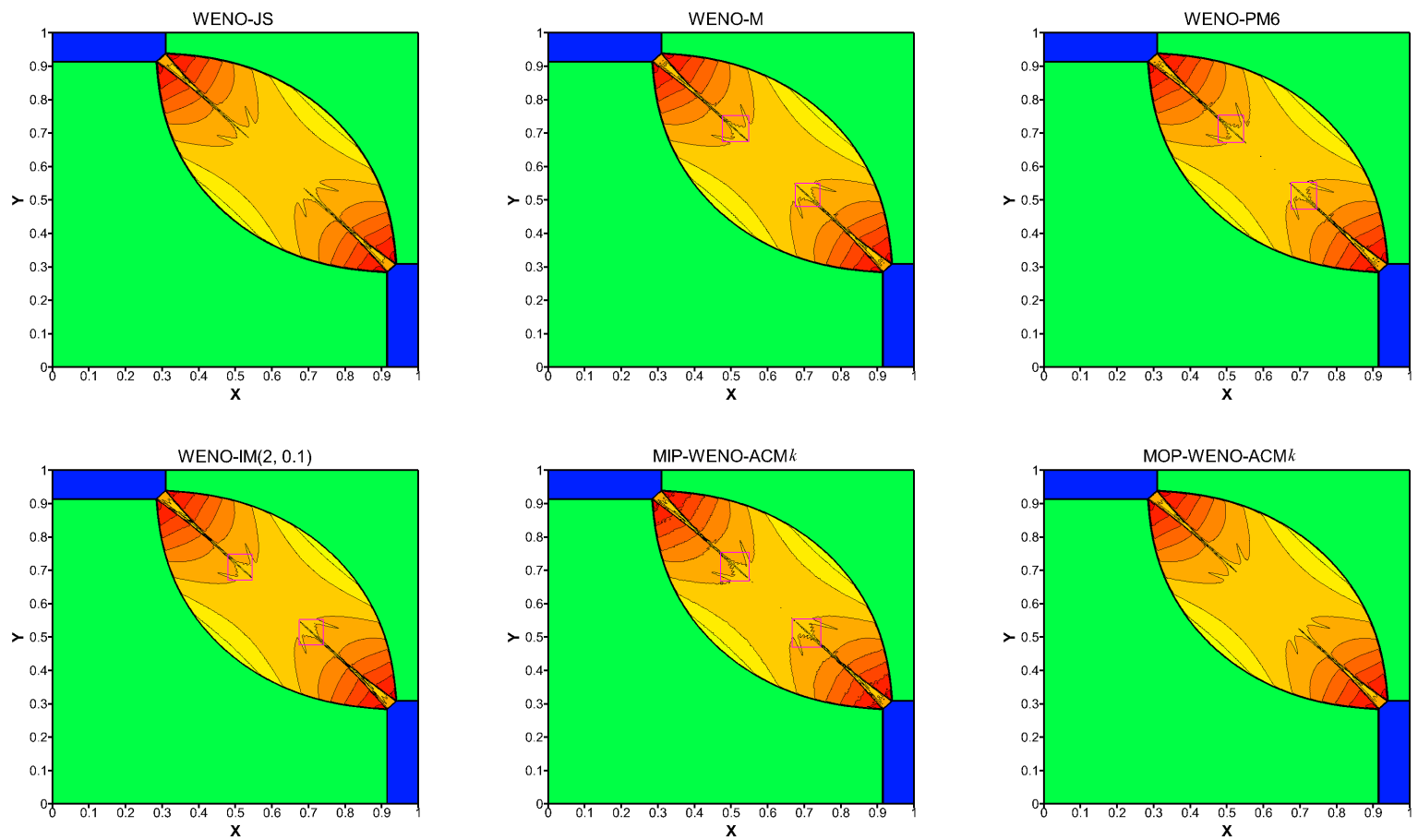

Fig. 22. Density plots for the $2 \mathrm{D}$ Riemann problem using 30 contour lines with range from 0.5 to 1.9 , computed using the WENO-JS, WENO-M, WENO-PM6, WENO-IM(2,.01), MIP-WENO-ACM $k$ and MOP-WENO-ACM $k$ schemes.
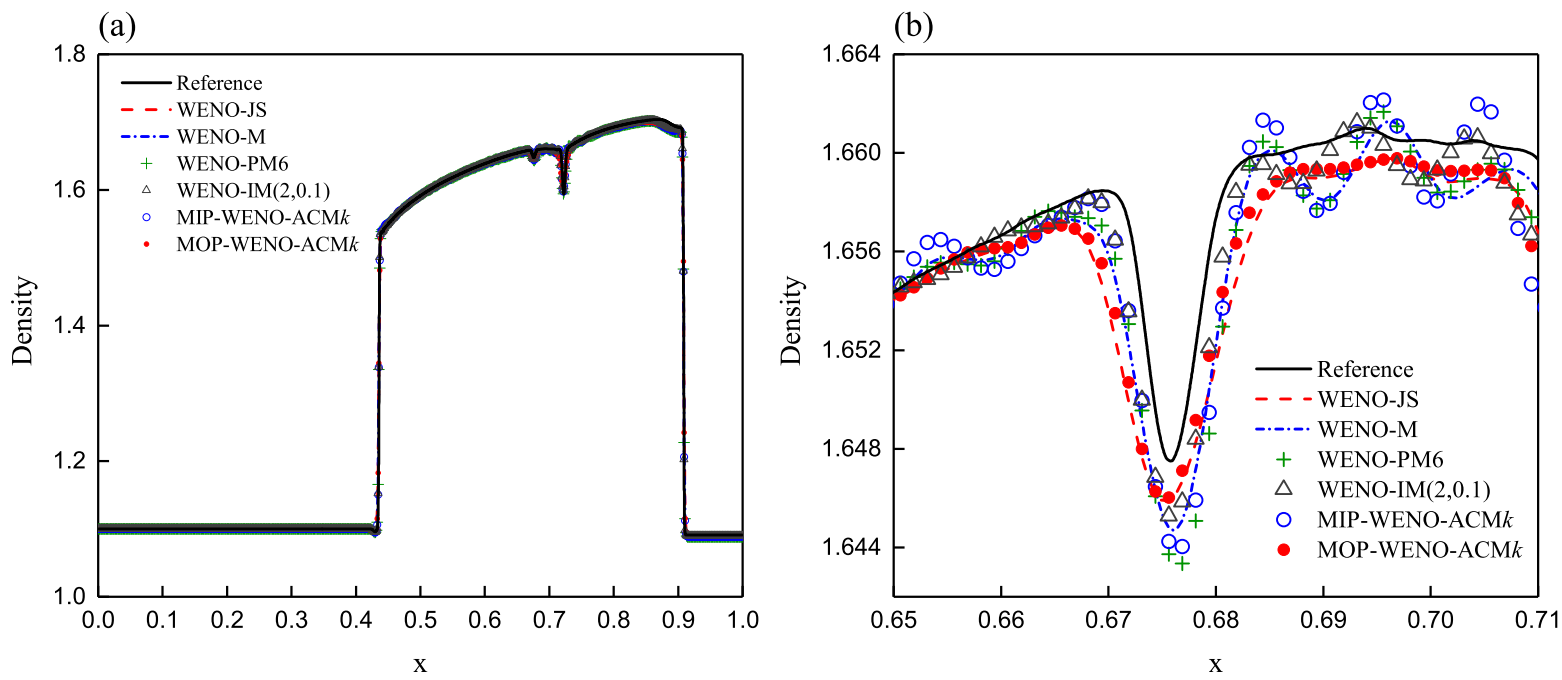

Fig. 23. The cross-sectional slices of density plot along the plane $y=0.5$, computed using the WENO-JS, WENO-M, WENO-PM6, WENO-IM (2, 0.1), MIP-WENO-ACM $k$ and MOP-WENO-ACM $k$ schemes. 

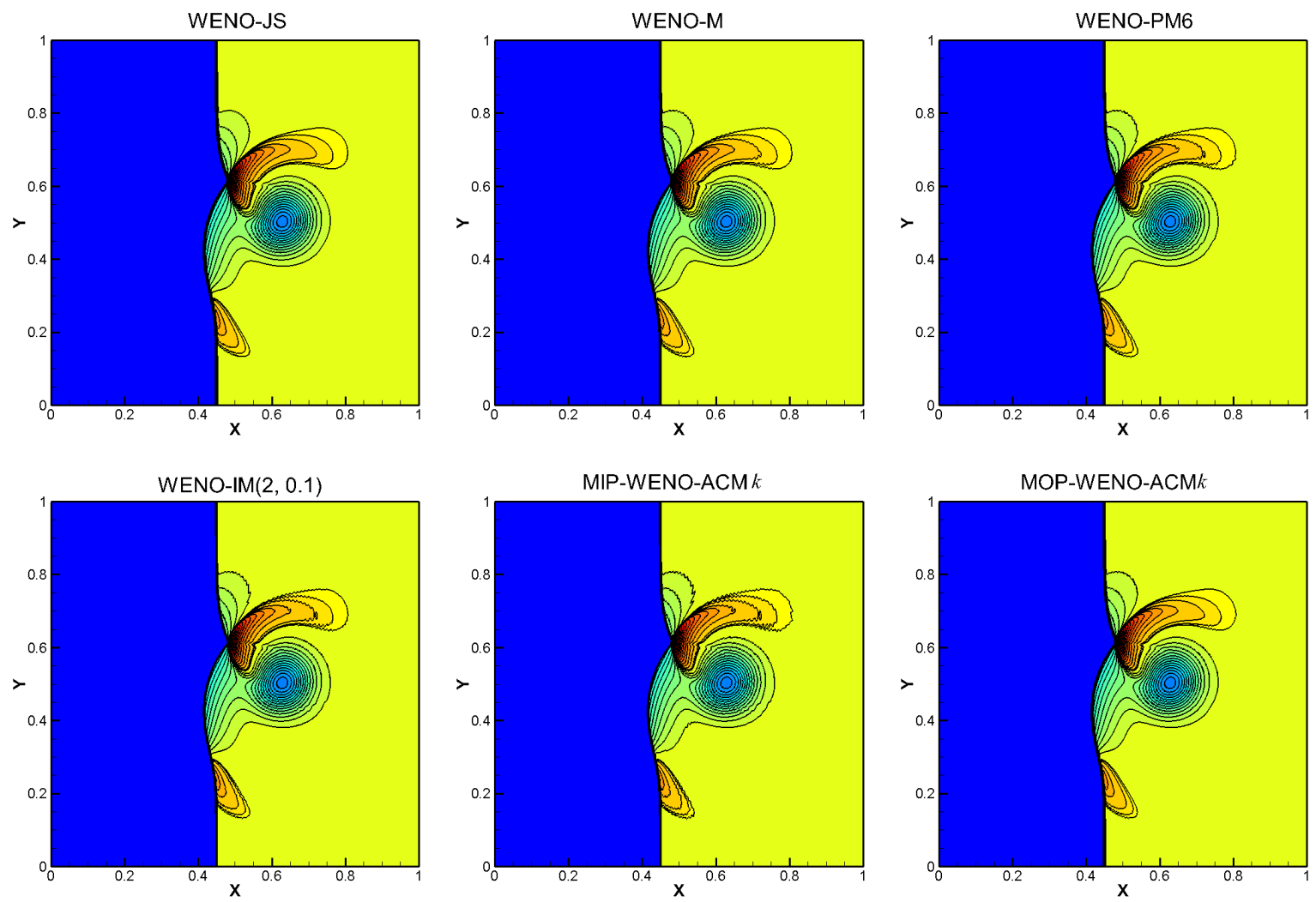

Fig. 24. Density plots for the Shock-vortex interaction using 30 contour lines with range from 0.9 to 1.4 , computed using the WENO-JS, WENO-M, WENO-PM6, WENO-IM(2,.01), MIP-WENO-ACM $k$ and MOP-WENO-ACM $k$ schemes.
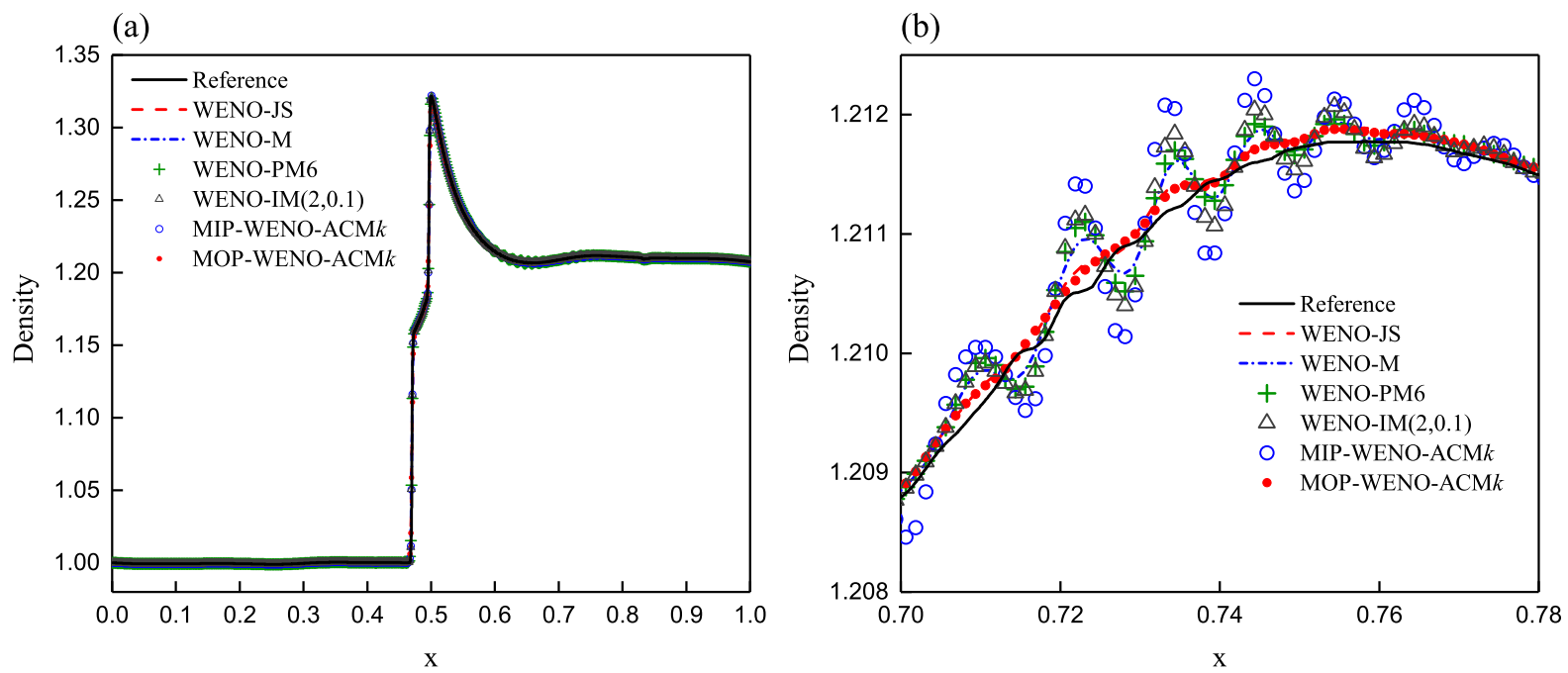

Fig. 25. The cross-sectional slices of density plot along the plane $y=0.65$, computed using the WENO-JS, WENO-M, WENO-PM6, WENO-IM (2, 0.1), MIP-WENO-ACM $k$ and MOP-WENO-ACM $k$ schemes. 


\section{References}

[1] F. Arandiga, A. Baeza, A.M. Belda, P. Mulet, Analysis of WENO schemes for full and global accuracy, SIAM J. Numer. Anal. 49 (2011) 893-915.

[2] R. Borges, M. Carmona, B. Costa, D.W. S., An improved weighted essentially non-oscillatory scheme for hyperbolic conservation laws, J. Comput. Phys. 227 (2008) 3101-3211.

[3] M. Castro, B. Costa, D.W. S., High order weighted essentially non-oscillatory WENO-Z schemes for hyperbolic conservation laws, J. Comput. Phys. 230 (2011) 1766-1792.

[4] A. Chatterjee, Shock wave deformation in shock-vortex interactions, Shock Waves 9 (1999) 95-105.

[5] W.S. Don, R. Borges, Accuracy of the weighted essentially non-oscillatory conservative finite difference schemes, J. Comput. Phys. 250 (2013) 347-372.

[6] P. Fan, Y.Q. Shen, B.L. Tian, A new smoothness indicator for improving the weighted essentially non-oscillatory scheme, J. Comput. Phys. 269 (2014) 329-354.

[7] H. Feng, F. Hu, R. Wang, A new mapped weighted essentially non-oscillatory scheme, J. Sci. Comput. 51 (2012) $449-473$.

[8] H. Feng, C. Huang, R. Wang, An improved mapped weighted essentially non-oscillatory scheme, Appl. Math. Comput. 232 (2014) $453-468$.

[9] S. Gottlied, C.W. Shu, Totalvariation diminishing runge-kutta schemes, Math. Comput. 67 (1998) 73-85.

[10] S. Gottlied, C.W. Shu, E. Tadmor, Strong stability-preserving high-order time discretization methods, SIAM Rev. 43 (2001) 89-112.

[11] A. Harten, ENO schemes with subcell resolution, J. Comput. Phys. 83 (1987) 148-184.

[12] A. Harten, B. Engquist, S. Osher, S. Chakravarthy, Uniformly high order essentially non-oscillatory schemes III, J. Comput. Phys. 71 (1987) 231-303.

[13] A. Harten, B. Osher, S. Engquist, S. Chakravarthy, Some results on uniformly high order accurate essentially non-oscillatory schemes, Appl. Numer. Math. 2 (1986) 347-377.

[14] A. Harten, S. Osher, Uniformly high order essentially non-oscillatory schemes I, SIAM J. Numer. Anal. 24 (1987) $279-309$.

[15] A.K. Henrick, T.D. Aslam, J.M. Powers, Mapped weighted essentially non-oscillatory schemes: Achieving optimal order near critical points, J. Comput. Phys. 207 (2005) 542-567.

[16] F. Hu, R. Wang, C. X., A modified fifth-order WENO-Z method hyperbolic conservation laws, J. Comput. Appl. Math. 303 (2016) $56-68$.

[17] G.S. Jiang, C.W. Shu, Efficient implementation of weighted ENO schemes, J. Comput. Phys. 126 (1996) $202-228$.

[18] P.D. Lax, X.D. Liu, Solution of two-dimensional Riemann problems of gas dynamics by positive schemes, SIAM J. Sci. Comput. 19 (1998) 319-340.

[19] R. Li, W. Zhong, A modified adaptive improved mapped WENO method, arXiv preprint (2020) arXiv:2011.03916.

[20] R. Li, W. Zhong, An efficient mapped WENO scheme using approximate constant mapping, arXiv preprint (2021) arXiv:2102.00231.

[21] Q. Liu, P. Liu, H. Zhang, Piecewise Polynomial Mapping Method and Corresponding WENO Scheme with Improved Resolution, Commun. Comput. Phys. 18 (2015) 1417-1444.

[22] X.D. Liu, S. Osher, T. Chan, Weighted essentially non-oscillatory schemes, J. Comput. Phys. 115 (1994) $200-212$.

[23] S.P. Pao, M.D. Salas, A numerical study of two-dimensional shock-vortex interaction, in: 14th Fluid and Plasma Dynamics Conference.

[24] S. Pirozzoli, Numerical methods for high-speed flows, Annu. Rev. Fluid Mech. 43 (2010) 163-194.

[25] Y.X. Ren, M. Liu, H. Zhang, A characteristic-wise hybrid compact-WENO scheme for solving hyperbolic conservation laws, J. Comput. Phys. 192 (2003) 365-386.

[26] R. Samala, G.N. Raju, A modified fifth-order WENO scheme for hyperbolic conservation laws, Comput. Math. Appl. 75 (2018) $1531-1549$.

[27] C.W. Schulz-Rinne, Classification of the Riemann problem for two-dimensional gas dynamics, SIAM J. Math. Anal. 24 (1993) $76-88$.

[28] C.W. Schulz-Rinne, J.P. Collins, H.M. Glaz, Numerical solution of the Riemann problem for two-dimensional gas dynamics, SIAM J. Sci. Comput. 14 (1993) 1394-1414.

[29] C.W. Shu, Essentially non-oscillatory and weighted essentially non-oscillatory schemes for hyperbolic conservation laws, in: Advanced Numerical Approximation of Nonlinear Hyperbolic Equations. Lecture Notes in Mathematics, volume 1697, Springer, Berlin, 1998, pp. $325-432$.

[30] C.W. Shu, S. Osher, Efficient implementation of essentially non-oscillatory shock-capturing schemes, J. Comput. Phys. 77 (1988) $439-471$.

[31] C.W. Shu, S. Osher, Efficient implementation of essentially non-oscillatory shock-capturing schemes II, J. Comput. Phys. 83 (1989) $32-78$.

[32] U.S. Vevek, B. Zang, T.H. New, A New Mapped WENO Method for Hyperbolic Problems, ICCFD10, in: Tenth International Conference on Computational Fluid Dynamics, Barcelona, Spain.

[33] U.S. Vevek, B. Zang, T.H. New, Adaptive mapping for high order WENO methods, J. Comput. Phys. 381 (2019) 162-188.

[34] R. Wang, H. Feng, C. Huang, A New Mapped Weighted Essentially Non-oscillatory Method Using Rational Function, J. Sci. Comput. 67 (2016) 540-580.

[35] R. Zhang, M. Zhang, C.W. Shu, On the order of accuracy and numerical performance of two classes of finite volume WENO schemes, Commun. Comput. Phys. 9 (2011) 807-827. 